\title{
CHERN-SIMONS THEORY, SURFACE SEPARABILITY, AND VOLUMES OF 3-MANIFOLDS
}

\author{
PIERRE DERBEZ, YI LIU, AND SHICHENG WANG
}

\begin{abstract}
We study the set $\operatorname{vol}(M, G)$ of volumes of all representations $\rho: \pi_{1} M \rightarrow$ $G$, where $M$ is a closed oriented 3-manifold and $G$ is either $\mathrm{Iso}_{+} \mathbf{H}^{3}$ or $\operatorname{Iso}_{e} \widetilde{\mathrm{SL}_{2}(\mathbb{R})}$.

By various methods, including relations between the volume of representations and the Chern-Simons invariants of flat connections, and recent results of surfaces in 3-manifolds, we prove that any 3-manifold $M$ with positive Gromov simplicial volume has a finite cover $\widetilde{M}$ with $\operatorname{vol}\left(\widetilde{M}, \mathrm{IsO}_{+} \mathbf{H}^{3}\right) \neq\{0\}$, and that any non-geometric 3-manifold $M$ containing at least one Seifert piece has a finite $\operatorname{cover} \widetilde{M}$ with $\operatorname{vol}\left(\widetilde{M}, \mathrm{Iso}_{e} \widehat{\mathrm{SL}_{2}(\mathbb{R})}\right) \neq\{0\}$.

We also find 3-manifolds $M$ with positive simplicial volume but $\operatorname{vol}\left(M, \mathrm{IsO}_{+} \mathbf{H}^{3}\right)=$ $\{0\}$, and non-trivial graph manifolds $M$ with $\operatorname{vol}\left(M, \mathrm{Iso}_{e} \widetilde{\mathrm{SL}_{2}(\mathbb{R})}\right)=\{0\}$, proving that it is in general necessary to pass to some finite covering to guarantee that $\operatorname{vol}(M, G) \neq\{0\}$.

Besides we determine vol $(M, G)$ when $M$ supports the Seifert geometry.
\end{abstract}

\section{CONTEnTS}

1. Introduction

2. Preliminaries

2.1. Topology of 3-manifolds after Thurston

2.2. Volume of representations of closed manifolds

3. Chern-Simons theory and additivity principle

3.1. Connections on principal bundles

3.2. Chern-Simons classes

3.3. Volume and Chern-Simons classes in Seifert geometry

3.4. Volume and Chern-Simons classes in hyperbolic geometry

3.5. Normal form near toral boundary of 3-manifolds

3.6. Additivity principle

4. Przytycki-Wise subsurfaces and separability

4.1. Merging finite covers

4.2. PW subsurfaces and partial PW subsurfaces

4.3. Virtual existence of partial PW subsurfaces

4.4. Virtual existence of corridor surfaces

5. Virtual extension of representations

5.1. Colored chunks and colored merging

5.2. Constructing the virtual representation

6. Volume computation

6.1. Virtually positive volume of representations

6.2. Volumes of representations of Seifert manifolds

7. Volumes of representations do not have the covering property

Date: January 3, 2014.

1991 Mathematics Subject Classification. 57M50, 51H20.

Key words and phrases. volume of representations, Chern-Simons invariants, surface separabilities. 
7.1. Non-trivial graph manifolds with vanishing Seifert volume 40

7.2. Mixed 3-manifolds with vanishing hyperbolic volume 41

8. Conclusions 41

References 42

\section{INTRODUCTION}

The volume of representations of 3-manifolds groups is a beautiful theory which has rich connections with many branches of mathematics. However the behavior of those volume functions seems still quite mysterious. To make our meaning more explicit, we first give some basic notions (which will be defined later) and properties of volume of representations. Let $N$ be a closed orientable 3 -manifold. Let $G$ be either $\operatorname{Iso}_{+} \mathbf{H}^{3} \cong \operatorname{PSL}(2 ; \mathbb{C})$, the orientation preserving isometry group of the hyperbolic 3-space, or $\mathrm{IsO}_{e} \widehat{\mathrm{SL}_{2}(\mathbb{R})} \cong$ $\mathbb{R} \times_{\mathbb{Z}} \widetilde{\mathrm{SL}_{2}(\mathbb{R})}$, the identity component of the isometry group of $\widetilde{\mathrm{SL}_{2}(\mathbb{R})}$. For each representation $\rho: \pi_{1} M \rightarrow G$, the volume of $\rho$ is denoted by $\operatorname{vol}_{G}(M, \rho)$.

Define

$$
\operatorname{vol}(M, G)=\left\{\operatorname{vol}_{G}(M, \rho) \text { when } \rho \text { runs over the representations } \pi_{1} M \rightarrow G\right\}
$$

Suppose $M$ supports a hyperbolic, respectively an $\widetilde{\mathrm{SL}_{2}(\mathbb{R})}$-geometry. Then $M$ naturally has its own hyperbolic volume $\operatorname{vol}_{\mathbf{H}^{3}}(M)$, respectively Seifert volume $\operatorname{vol}_{\mathrm{SL}_{2}(\mathbb{R})}(M)$. We denote by $\|M\|$ the Gromov norm of $M$, which measures, up to a multiplicative constant, the total hyperbolic volume of the hyperbolic pieces of $M[\mathrm{Gr}, \mathrm{So}]$. The following theorem contains some known basic results of the theory of volume representations. For its development, see [BG1, BG2, Re] and their references.

Theorem 1.1. Let $N$ be a closed orientable 3-manifold.

(1) Both $\operatorname{vol}(N, \mathrm{PSL}(2 ; \mathbb{C}))$ and $\operatorname{vol}\left(N, \mathrm{Iso}_{e} \widehat{\mathrm{SL}_{2}(\mathbb{R})}\right)$ contain at most finitely many values. Hence the supremums $\operatorname{HV}(N)$ and $\operatorname{SV}(N)$ of $\operatorname{vol}(N, \operatorname{PSL}(2 ; \mathbb{C}))$ and $\operatorname{vol}\left(N, \mathrm{IsO}_{e} \widetilde{\mathrm{SL}_{2}(\mathbb{R})}\right)$ are reached.

(2) If $N$ admits a hyperbolic geometric structure, then $\mathrm{HV}(N)$ equals $\operatorname{vol}_{\mathbf{H}^{3}}(N)$, reached by any discrete and faithful representation. A similar statement holds when $N$ admits an $\widetilde{\mathrm{SL}_{2}(\mathbb{R})}$ geometric structure.

(3) $\mathrm{HV}(N) \leq \mu_{3}\|N\|$, where $\mu_{3}$ denotes the volume of any ideal regular tetrahedron in $\mathbf{H}^{3}$.

(4) Let $f: M \rightarrow N$ be a map between closed orientable 3-manifolds and let $\rho$ : $\pi_{1} N \rightarrow G$ denote a representation. Then

$$
\operatorname{vol}_{G}\left(M, f^{*} \rho\right)=\operatorname{deg}(f) \operatorname{vol}_{G}(N, \rho) .
$$

Hence,

$$
\mathrm{HV}(M) \geq|\operatorname{deg} f| \operatorname{HV}(N) \text { and } \mathrm{SV}(M) \geq|\operatorname{deg} f| \operatorname{SV}(N) .
$$

We call $\mathrm{HV}(N)$ and $\mathrm{SV}(N)$ in the conclusion of Theorem 1.1(1) the hyperbolic volume and the Seifert volume of $N$, respectively.

Remark 1.2. Let $M$ and $N$ be two closed oriented 3-dimensional manifolds and let $D(M, N)$ be the set of degrees of maps from $M$ to $N$. Let $\mathcal{D}$ be the set of all closed orientable 3-manifolds $N$ with $D(M, N)$ finite for any fixed $M$. By Theorem 1.1 (4), 
$\mathrm{SV}(N)=\operatorname{HV}(N)=0$ if $N \notin \mathcal{D}$. It is known that (see [DSW] for example), $N \in \mathcal{D}$ if and only if $N$ contains a prime factor $Q$ with non-trivial geometric decomposition, or supporting an $\widetilde{\mathrm{SL}_{2}(\mathbb{R})}$ or a hyperbolic geometry. This fact combined with Theorem 1.1 (2), (3), (4) implies that if $\operatorname{vol}\left(N, \mathrm{Iso}_{\mathrm{e}} \widehat{\mathrm{SL}_{2}(\mathbb{R})}\right) \neq\{0\}$ then necessarily a prime factor of $N$ has a non-trivial geometric decomposition, or supports an $\widetilde{\mathrm{SL}_{2}(\mathbb{R})}$ or a hyperbolic geometry and if $\operatorname{vol}(N, \operatorname{PSL}(2 ; \mathbb{C})) \neq\{0\}$ then necessarily a prime factor of $N$ contains some hyperbolic JSJ pieces.

Besides Theorem 1.1, Thurston pointed out the relation between Chern-Simons invariants and the hyperbolic volume of hyperbolic 3-manifolds for discrete and faithful representations [Th2]. Such a relation is extended by Kirk-Klassen [KK] for cusped hyperbolic 3 -manifolds and discrete and faithful representations into $\operatorname{PSL}(2 ; \mathbb{C})$, and by Khoi [Kh] for closed manifolds with the group $\widetilde{\mathrm{SL}_{2}(\mathbb{R})}$ (as a subgroup of $\mathrm{IsoSL}_{2}(\mathbb{R})$ ).

Despite those significant results, the answer to the questions below, which is a main motivation of this paper, seems still remarkably unknown. Recall that a non-negative invariant $\eta$ of 3-manifolds is said to satisfy the covering property in the sense of Thurston, if for any finite covering $p: \widetilde{N} \rightarrow N$, we have $\eta(\widetilde{N})=|\operatorname{deg}(p)| \eta(N)$.

Question 1. Let $M$ be a closed 3-manifold and let $G$ be either $\operatorname{PSL}(2, \mathbb{C})$ or $\operatorname{Iso}_{e} \widetilde{\mathrm{SL}_{2}(\mathbb{R})}$.

(1) (a) How to find non-zero elements in $\operatorname{vol}(M, G)$ ?

(b) More weakly, how to find non-zero elements in $\operatorname{vol}(\widetilde{M}, G)$ for some finite cover $\widetilde{M}$ of $M$ ?

(2) Does HV or SV satisfy the covering property?

Remark 1.3. Three-manifold invariants with the covering property was first addressed by Thurston in the 1970s [Ki, Problem 3.16(A)]. The simplicial volume has the covering property (See Gromov, Thurston, Soma [Gr, Th1, So], an early evidence of such application appears in Milnor-Thurston [MT]). Some papers define invariants with the covering property for graph manifolds, say [WW, LW,, $\mathrm{Ne}]$, but each one vanishes for some graph manifolds.

So far it seems that we only know that HV, respectively, SV, satisfies the covering property for the hyperbolic, respectively, Seifert manifolds. In hyperbolic geometry this property comes from the relation between the simplicial volume and HV. In Seifert geometry one can compute SV in terms of the Euler classes of the Seifert manifold and the Euler characteristic of its orbifold and these invariants behave naturally under covering maps.

The results of Brooks-Goldman [BG2] and Eisenbud-Hirsch-Neumann [EHN] allow us to describe the set vol $\left(M, \mathrm{Iso}_{\mathrm{e}} \widehat{\mathrm{SL}_{2}(\mathbb{R})}\right)$ for each closed 3-manifold $M$ supporting an $\widetilde{\mathrm{SL}_{2}(\mathbb{R})}$-geometry. It is known that $M$ supports an $\widetilde{\mathrm{SL}_{2}(\mathbb{R})}$-geometry if and only if $M$ is a Seifert manifold with non-zero Euler number $e(M)$ over an orbifold of negative Euler characteristic. We use $\llcorner a\lrcorner$ and $\ulcorner a\urcorner$ for $a \in \mathbb{R}$ to denote respectively, the greatest integer less than or equal to $a$ and the least integer greater than or equal to $a$.

Proposition 1.4. Suppose $M$ supports an $\widetilde{\mathrm{SL}_{2}(\mathbb{R})}$-geometry and that its base 2-orbifold has a positive genus $g$. Then

$$
\operatorname{vol}\left(M, \mathrm{Iso}_{\mathrm{e}} \widetilde{\mathrm{SL}_{2}(\mathbb{R})}\right)=\left\{\frac{4 \pi^{2}}{|e(M)|}\left(\sum_{i=1}^{r}\left(\frac{n_{i}}{a_{i}}\right)-n\right)^{2}\right\}
$$


where $n_{1}, \ldots, n_{r}, n$ are integers such that

$$
\sum_{i=1}^{r}\left\llcorner n_{i} / a_{i}\right\lrcorner-n \leq 2 g-2, \quad \sum_{i=1}^{r}\left\ulcorner n_{i} / a_{i}\right\urcorner-n \geq 2-2 g
$$

and $a_{1}, \ldots, a_{r}$ are the indices of the singular points of the orbifold of $M$.

Remark 1.5. In order to check Proposition 1.4, we will describe all representations with non-zero volume. Proposition 1.4 presents explicitly the rationality of the elements in $\operatorname{vol}\left(M, \mathrm{Iso}_{\mathrm{e}} \widetilde{\mathrm{SL}_{2}(\mathbb{R})}\right)$, which was proved by Reznikov [Re].

As a partial answer to Question 1 (1) for non-geometric manifolds, it was known recently that each non-trivial graph manifold $M$ has a finite cover $\widetilde{M}$ such that

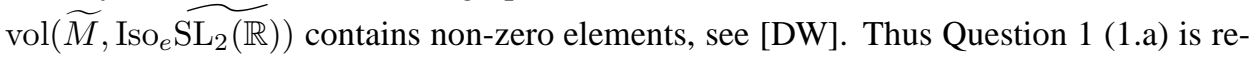
duced to the non-geometric 3-manifolds containing hyperbolic JSJ pieces (the so-called mixed 3-manifold). In view of Theorem[1.1 (2) (3), as well as the result of [DW], and in an attempt to seal a relation between the Gromov simplicial volume and the hyperbolic volume, M. Boileau and several others wondered the following more direct version of Question 1 (1):

Question 2. Suppose that the Gromov norm $\|M\|$ is positive.

(1) Is there a representation $\rho: \pi_{1} M \rightarrow \operatorname{PSL}(2 ; \mathbb{C})$ with positive volume?

(2) More weakly is there a representation $\rho: \pi_{1} \widetilde{M} \rightarrow \operatorname{PSL}(2 ; \mathbb{C})$ with positive volume for some finite covering $\widetilde{M}$ of $M$ ?

From now on $M$ will always be assumed to be a closed oriented irreducible nongeometric 3-manifold.

The main results of this paper are the following two theorems, which answer Questions 1 and 2 respectively.

Theorem 1.6. Suppose that $M$ is a closed oriented irreducible non-geometric 3-manifold.

(1) If $M$ contains at least one hyperbolic geometric piece, then the hyperbolic volume of some finite cover $\tilde{M}$ is positive.

(2) If M contains at least one Seifert geometric piece, then the Seifert volume of some finite cover $\tilde{M}$ is positive.

\section{Theorem 1.7.}

(1) There are closed oriented non-trivial graph manifolds with vanishing Seifert volume.

(2) There are closed oriented irreducible 3-manifolds with non-vanishing Gromov norm but vanishing hyperbolic volume.

Corollary 1.8. Neither the hyperbolic volume nor the Seifert volume have the covering property.

Now let us have a brief discussion of our proofs of the main results.

The difficulty of Question 1 can more or less be seen from the definition: to get a nonzero element in $\operatorname{vol}(M, G)$ we need first to find an a priori "significant" representation $\rho: \pi_{1} M \rightarrow G$, and then to be able to compute its volume. In the geometric case, there is a natural significant representation given by the faithful and discrete representation of its fundamental group in the Lie group of its geometry. In the non-geometric case one might think to use the geometry of its pieces to construct a global significant representation. 
However in this new situation many problems occur: First the geometric pieces have nonempty boundary and the volume of representation is not easy to manipulate and moreover we must make sure that the local representations are compatible in the toral boundaries in order to be glued together. Then another problem arises when we want to compute the volume of a global representation from the local volumes. Can we add the volumes of those pieces to get the volume of the presentation?

In order to prove Theorem 1.6 and Theorem 1.7, we will first consider the volume of representations from the perspective of Chern-Simons theory and prove the so-called additivity principle.

Denote by $G$ the semi-simple Lie group $\operatorname{Iso}_{e} \widetilde{\mathrm{SL}_{2}(\mathbb{R})}$ or $\operatorname{PSL}(2, \mathbb{C})$ with the associated Riemannian homogeneous spaces $X$ which is $\widetilde{\mathrm{SL}_{2}(\mathbb{R})}$ or $\mathbf{H}^{3}$ endowed with the closed $G$-invariant volume form $\omega_{X}$.

Denote by $\mathfrak{g}$ the Lie algebra of $G$. We recall (see Section 3 for more details) that the Chern-Simons classes with structure group $\operatorname{PSL}(2, \mathbb{C})$ are based on the first Pontrjagin class and in the same way we define the Chern-Simons classes with structure group $\mathrm{Iso}_{e} \widetilde{\mathrm{SL}_{2}(\mathbb{R})}$ based on the invariant polynomial defined by $\mathbf{R}(A \otimes A)=\operatorname{Tr}\left(X^{2}\right)+t^{2}$ where $A$ is an element of the Lie algebra of $\mathrm{Iso}_{e} \widetilde{\mathrm{SL}_{2}(\mathbb{R})}$ which decomposes into $X+t$ where $X$ is in the Lie algebra of $\widetilde{\mathrm{SL}_{2}(\mathbb{R})}$ and $t \in \mathbb{R}$. Denote the imaginary part of the complex number $z$ by $\Im(z)$.

Proposition 1.9. Let $\rho$ be a representation of $\pi_{1} M$ into $G$ and $A$ be a corresponding flat $G$-connection in the principal bundle $P=M \times_{\rho} G$. Suppose that $P$ admits a section $\delta$ over $M$.

(1) If $G$ equals $\mathrm{Iso}_{e} \widetilde{\mathrm{SL}_{2}(\mathbb{R})}$ then

$$
\mathfrak{c s}_{M}(A, \delta)=\int_{M} \delta^{*} \mathbf{R}\left(\mathrm{d} A \wedge A+\frac{1}{3} A \wedge[A, A]\right)=\frac{2}{3} \operatorname{vol}_{G}(M, \rho) .
$$

In particular, the Chern-Simons invariant of flat $\mathrm{Iso}_{e} \widetilde{\mathrm{SL}_{2}(\mathbb{R})}$-connections is gauge invariant.

(2) If $G$ equals $\operatorname{PSL}(2 ; \mathbb{C})$ then

$$
\Im\left(\mathfrak{c s}_{M}(A, \delta)\right)=-\frac{1}{\pi^{2}} \operatorname{vol}_{G}(M, \rho) .
$$

Remark 1.10. Assuming that $P=M \times{ }_{\rho} G$ admits a section in Proposition1.9(1) means equivalently that $\rho$ admits a lift into $\widehat{\mathrm{SL}_{2}(\mathbb{R})}$ so that the bundle admits a reduction to an $\widehat{\mathrm{SL}_{2}(\mathbb{R})}$-bundle and we reckon that the correspondence for $G=\widetilde{\mathrm{SL}_{2}(\mathbb{R})}$ is pointed in $[\mathrm{Re}]$, and verified in [Kh] by a long and subtle computation. However for our own understanding we reprove it in a very simple way underscoring that the correspondence is quite natural and comes directly from the structural equations of the Lie group involved (Section 3.3). The correspondence in Proposition [1.9 (2) is derived from [KK], using a formula established by Yoshida in [Yo] (Section 3.4). The imaginary part of the Chern-Simons invariants of flat $\operatorname{PSL}(2 ; \mathbb{C})$-connections is gauge invariant from the formula for it does not depend on the chosen section.

Then from the representations in normal form developed in [KK], we have the so-called additivity principle, that is, we can add the volumes of those pieces to get the globe volumes. We state it in the following 
Theorem 1.11. Let $M$ be an irreducible oriented closed 3-manifold with JSJ tori $T_{1}, \cdots, T_{r}$ and JSJ pieces $J_{1}, \cdots, J_{k}$, and let $\zeta_{1}, \cdots, \zeta_{r}$ be slopes on $T_{1}, \cdots T_{r}$, respectively.

Suppose that $G$ is either $\mathrm{IsO}_{e} \widetilde{\mathrm{SL}_{2}(\mathbb{R})}$ or $\operatorname{PSL}(2 ; \mathbb{C})$, and that

$$
\rho: \pi_{1}(M) \rightarrow G
$$

is a representation vanishing on the slopes $\zeta_{i}$, and that $\widehat{\rho}_{i}: \pi_{1}\left(\widehat{J}_{i}\right) \rightarrow G$ are the induced representations, where $\widehat{J}_{i}$ is the Dehn filling of $J_{i}$ along slopes adjacent to its boundary, with the induced orientations. Then

$$
\operatorname{vol}_{G}(M, \rho)=\operatorname{vol}_{G}\left(\widehat{J}_{1}, \widehat{\rho}_{1}\right)+\operatorname{vol}_{G}\left(\widehat{J}_{2}, \widehat{\rho}_{2}\right)+\ldots+\operatorname{vol}_{G}\left(\widehat{J}_{k}, \widehat{\rho}_{k}\right) .
$$

With Proposition 1.9 and Theorem 1.11 at hands, for a given 3-manifold $M$ satisfying the conditions of Theorem 1.6, how do we contstruct a finite cover $\tilde{M}$ of $M$ and a representation $\rho: \pi_{1}(\tilde{M}) \rightarrow G$ with positive volume? Such a cover $\tilde{M}$ and a representation $\rho$ are not difficult to describe up to some finite cover and conjugation as we see below.

The prime picture of the cover $\tilde{M}$ is simple. Fix a JSJ piece $J$ of $M$ with the right geometry. The regular finite cover $\tilde{M}$ of $M$ can be cut along some of its JSJ tori into three parts: Part 1 are some disjoint preimage components (elevations) of $J$, Part 2 some "corridors", and Part 3 the remaining part; components in Part 1 and Part 3 are connected by those corridors in Part 2. The crucial property of corridors is that in each corridor $X$ there is a corridor surface $R$, so that if $X$ is a corridor connecting some $\tilde{J}$ in Part 1 through a component $\tilde{T}$ of $\partial \tilde{J}$, then $\partial R$ has exactly one component (circular doorsill) in $\tilde{T}$.

It seemed hopeless to find such corridors in general, until the recent striking results of surfaces in 3-manifolds due to Wise and his co-authors [HW, Wi, PW1, PW2, PW3]. In this paper we will construct such corridors (Theorem 4.12) from what we call parallelcutting partial Przytycki-Wise subsurfaces (Theorem 4.11), then we will merge the three parts above to provide the designed finite cover $\tilde{M}$ (Proposition 4.2, Corollary 4.5). To understand the key issue that we will address, consider the following situation: If $S$ is a $\pi_{1}$ injectively immersed, virtually embedded, connected, closed subsurface of $M$, and if $T$ is a JSJ torus of $M$, is there a regular finite cover $\tilde{M}$ of $M$ such that any elevation of $S$ intersects any elevation of $T$ in at most one connected component? Generally speaking, the answer seems to be negative when $S$ and $T$ intersects in more than one component. In particular, the double separability between $S$ and $T$ does not automatically lead to such a cover $\tilde{M}$. However, the main new input of Theorem 4.12 is the parallel cutting condition, which morally assumes that $S$ intersects every JSJ torus of $M$ in virtually parallel components (Definition 4.10). Under this and other suitable working assumptions, we will be able to resolve the key issue by applying the separability criterion of Rubinstein-Wang [RW] in graph submanifolds of $M$ and the relatively quasiconvex separability of Wise [Wi] in hyperbolic pieces.

Thus the term "surface separability" in the title of this paper mostly refers to virtual simplification of intersection between an immersed subsurface and any JSJ torus, rather than intersection of an immersed subsurface with itself.

We describe the representation $\rho: \pi_{1}(\tilde{M}) \rightarrow G$ (Theorem 5.2) as follows: $\rho \mid$ on the group of each component $\tilde{J}$ of Part 1 factors through a "mighty and natural" representation of the $\pi_{1}(\widehat{J})$, where $\widehat{J}$ is the closed 3-manifold obtained by Dehn filling of $\tilde{J}$ along its doorsills and the representation is discrete and faithful (the corridor surfaces can be chosen to be so); $\rho \mid$ on the group of each component of Part 3 is trivial; and $\rho \mid$ on the group of 
each corridor is based on the crucial property of its corridor surface and is given by the homological pairing $\pi_{1}(X) \longrightarrow H_{1}(X ; \mathbb{Z}) \stackrel{[R]}{\longrightarrow} \mathbb{Z} \longrightarrow G$, where everything is oriented, $[R] \in H_{2}(X, \partial X ; \mathbb{Z}) \cong H^{1}(X ; \mathbb{Z})$. The global representation $\rho$ can be obtained by gluing together the local representations $\rho \mid$ provided they induce conjugate representations on each boundary components of the Parts 1, 2 and 3 (Lemma 3.2 or Remark 5.5). To make sure that the local representations are conjugate in the boundary, we need the concepts of colored chunks and colored merging, where colored chunks can be merged with matching color in a further finite cover (Lemma 5.3) and also certain so called "class invertible properties" of the groups $\mathrm{Iso}_{e} \mathrm{SL}_{2}(\mathbb{R})$, and $\operatorname{PSL}(2 ; \mathbb{C})$ (Lemma6.1). Finally the conjugacy issue above will be managed in Lemma 5.4

To prove Theorem 1.7 some arguments in [EHN], an example of Motegi [Mo] and a result of Hoffman-Matignon $[\mathrm{HM}]$ are also used.

The organization of this paper is reflected by the table of contents. Efforts have been made in organizing the materials so that our results can be verified smoothly, and readers can access the topic more easily.

Acknowledgement. We thank Professor Michel Boileau and Professor Daniel Matignon for helpful communications.

\section{PRELIMINARIES}

In this section, we review the geometric decomposition of 3-manifolds and volume of representations of closed manifolds.

2.1. Topology of 3-manifolds after Thurston. Let $N$ be a connected compact prime orientable 3-manifold with toral or empty boundary.

2.1.1. Geometric decomposition. As consequence of the geometrization of 3-manifolds [Th1, Th2] achieved by G. Perelman and W. Thurston, exactly one of the following holds:

- Either $N$ is geometric, supporting one of the following eight geometries: $\mathbf{H}^{3}$, $\widetilde{\mathrm{SL}_{2}(\mathbb{R})}, \mathbf{H}^{2} \times \mathbb{R}$, Sol, Nil, $\mathbb{R}^{3}, \mathbf{S}^{3}$ and $\mathbf{S}^{2} \times \mathbb{R}$ (where $\mathbf{H}^{n}, \mathbb{R}^{n}$ and $\mathbf{S}^{n}$ are the $n$ dimensional hyperbolic space, Euclidean space, and spherical space respectively);

- or $N$ has a canonical nontrivial geometric decomposition. In other words, there is a nonempty minimal union $\mathcal{T}_{N} \subset N$ of disjoint essential tori and Klein bottles of $N$, unique up to isotopy, such that each component of $N \backslash \mathcal{T}_{N}$ is either Seifert fibered or atoroidal. In the Seifert fibered case the piece supports the $\mathbf{H}^{2} \times \mathbb{R}$ geometry and the $\widetilde{\mathrm{SL}_{2}(\mathbb{R})}$ geometry, and in the atoroidal case the piece supports the $\mathbf{H}^{3}$ geometry.

When $N$ has nontrivial geometric decomposition, we call the components of $N \backslash \mathcal{T}_{N}$ the geometric pieces of $N$, or more specifically, Seifert pieces or hyperbolic pieces according to their geometry. We call $N$ a mixed 3-manifold if $N$ contains at least one hyperbolic piece, or a graph manifold otherwise.

A chunk of $N$ is a submanifold that is a union of a subset of geometric pieces, glued up along the cut tori between them. A graph chunk is a chunk which is a graph manifold.

Traditionally, there is another decomposition introduced by Jaco-Shalen [JS] and Johannson [Joh], known as the JSJ decomposition. When $N$ contains no essential Klein bottles and has a nontrivial geometric decomposition, the JSJ decomposition of $N$ coincides with its geometric decomposition, so the cut tori and the geometric pieces may be referred to as the JSJ tori and the JSJ pieces, respectively. Possibly after passing to a 
double cover of $N$, we may assume that $N$ contains no essential Klein bottle. In fact, the following lemma of virtual reduction is well known, (cf. [PW3, Lemma 3.1]).

Lemma 2.1. If $N$ is a closed prime 3-manifold which is not geometric, then there exists a regular finite covering $\tilde{N}$ of $N$ satisfying the following:

- $\tilde{N}$ contains no essential Klein bottle;

- each JSJ torus of $\tilde{N}$ is adjacent to a pair of distinct JSJ pieces;

- each Seifert piece is a product of $S^{1}$ and a compact surface of positive genus.

2.1.2. Hyperbolic pieces. A hyperbolic piece $J$ can be realized as a complete hyperbolic 3 -manifold of finite volume, unique up to isometry by Mostow Rigidity. With respect to the geometry, any properly $\pi_{1}$-injectively immersed connected subsurface $j: S \rightarrow$ $J$ of finite type, is either geometrically finite or geometrically infinite, unless $\pi_{1}(S)$ is elementary (trivial or infinite cyclic). Geometrically infinite subsubsurfaces are virtual fibers; geometrically finite subsurfaces are quasi-Fuchsian so any conjugate of $\pi_{1}(S)$ in $\pi_{1}(J)$ is quasiconvex relatively to the cusps. It follows from the work of Wise [Wi] that $\pi_{1}(S)$ is separable in $\pi_{1}(J)$ in the cusped case, and the closed case follows from Agol's proof of the Virtual Haken Conjecture $[\overline{\mathrm{Ag}}]$. In particular, $S$ is virtually embedded in $J$.

We briefly recall the Thurston's Hyperbolic Dehn Filling Theorem. Let $J$ denote a compact, orientable 3 -manifold whose boundary consists of tori $T_{1}, \ldots, T_{p}$ and whose interior admits a complete hyperbolic metric. We denote by $J_{\max }$ the interior of $M$ with a system of maximal cusps removed. Identify $J$ with $J_{\max }$, then $\partial J$ has a Euclidean metric induced from the hyperbolic metric and each closed Euclidean geodesic in $\partial J$ has the induced length. The Hyperbolic Dehn Filling Theorem [Th1, Theorem 5.8.2] can be stated in the following form.

Theorem 2.2. Let $J$ be a compact oriented 3-manifold with toral boundary $T_{1} \cup \ldots \cup T_{p}$ whose interior admits a complete hyperbolic structure. Identify $J$ with $J_{\max }$. Then there is a positive constant $C$ such that the the closed 3-manifold $J\left(\zeta_{1}, \ldots, \zeta_{n}\right)$ obtained by Dehn filling each $T_{i}$ along $\zeta_{i}$ admits a complete hyperbolic structure if each slope $\zeta_{i}$ has then length greater than $C$. Moreover, with suitably chosen base points, $J\left(\zeta_{1}, \ldots, \zeta_{n}\right)$ converges to $J$ in the Gromov-Hausdorff sense as the minimal length of $\zeta_{i}$ tends to infinity.

2.1.3. Seifert pieces. A Seifert piece $J$ of a non-geometric prime closed 3-manifold $N$ supports both the $\mathbf{H}^{2} \times \mathbb{R}$ geometry and the $\widehat{\mathrm{SL}_{2}(\mathbb{R})}$ geometry. In this paper we are more interested in the latter, so we describe the structure of $\widetilde{\mathrm{SL}_{2}(\mathbb{R})}$ geometric manifolds in the following.

We consider the group $\operatorname{PSL}(2 ; \mathbb{R})$ as the orientation preserving isometries of the hyperbolic 2 -space $\mathbf{H}^{2}=\{z \in \mathbb{C}, \Im(z)>0\}$ with $i$ as a base point. In this way $\operatorname{PSL}(2 ; \mathbb{R})$ is a (topologically trivial) circle bundle over $\mathbf{H}^{2}$. Denote by $p: \widetilde{\operatorname{SL}_{2}(\mathbb{R})} \rightarrow \operatorname{PSL}(2 ; \mathbb{R})$ the universal covering of $\operatorname{PSL}(2 ; \mathbb{R})$ with the induced metric. Then $\widetilde{\mathrm{SL}_{2}(\mathbb{R})}$ is a line bundle over $\mathbf{H}^{2}$. For any $\alpha \in \mathbb{R}$, denote by $\operatorname{sh}(\alpha)$ the element of $\widetilde{\mathrm{SL}_{2}(\mathbb{R})}$ whose projection into $\operatorname{PSL}(2 ; \mathbb{R})$ is given by $\left(\begin{array}{cc}\cos (2 \pi \alpha) & \sin (2 \pi \alpha) \\ -\sin (2 \pi \alpha) & \cos (2 \pi \alpha)\end{array}\right)$. Then the set $\{\operatorname{sh}(n), n \in \mathbb{Z}\}$, is the kernel of $p$ as well as the center of $\widetilde{\mathrm{SL}_{2}(\mathbb{R})}$, acting by integral translation along the fibers of $\widetilde{\mathrm{SL}_{2}(\mathbb{R})}$. By extending this $\mathbb{Z}$-action on the fibers by the $\mathbb{R}$-action we get the whole identity component of the isometry group of $\widehat{\mathrm{SL}_{2}(\mathbb{R})}$. To summarize we have the 
following diagram of central extensions

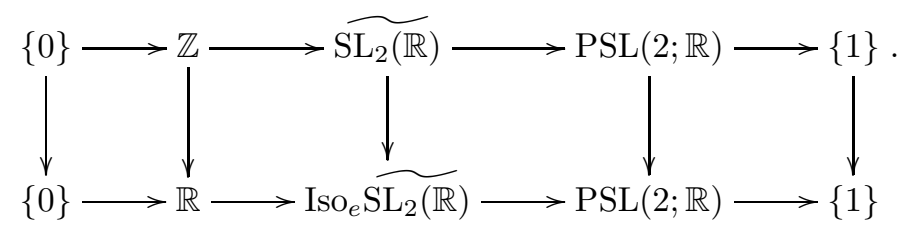

In particular the group $\mathrm{IsO}_{e} \widehat{\mathrm{SL}_{2}(\mathbb{R})}$ is generated by $\widehat{\mathrm{SL}_{2}(\mathbb{R})}$ and the image of $\mathbb{R}$ which intersect together in the image of $\mathbb{Z}$, where each element $x$ on $\mathbb{R}$ is naturally identified with the translation $\tau_{x}$ of length $x$. More precisely we state the following useful lemma which is easy to check.

Lemma 2.3. We have the identification $\mathrm{Iso}_{e} \widehat{\mathrm{SL}_{2}(\mathbb{R})}=\mathbb{R} \times_{\mathbb{Z}} \widehat{\mathrm{SL}_{2}(\mathbb{R})}$ : where $(x, h) \sim$ $\left(x^{\prime}, h^{\prime}\right)$ if and only if there exists an integer $n \in \mathbb{Z}$ such that $x^{\prime}-x=n$ and $h^{\prime}=$ $\operatorname{sh}(-n) \circ h$.

Let $F_{g, n}$ be an oriented $n$-punctured surface of genus $g \geq 0$ with boundary components $s_{1}, \ldots, s_{n}$ with $n \geq 0$. Then $J^{\prime}=F_{g, n} \times \mathbf{S}^{1}$ is oriented if $\mathbf{S}^{1}$ is oriented. Let $h_{i}$ be the oriented $\mathbf{S}^{1}$-fiber on the torus $T_{i}=s_{i} \times h_{i}$. We say that $\left(s_{i}, h_{i}\right)$ is a section-fiber basis of $T_{i}$. Let $0 \leq s \leq n$. Now attach $s$ solid tori $V_{i}$ 's to the boundary tori $T_{i}$ 's of $J^{\prime}$ such that the meridian of $V_{i}$ is identified with the slope $a_{i} s_{i}+b_{i} h_{i}$ where $a_{i}>0,\left(a_{i}, b_{i}\right)=1$ for $i=$ $1, \ldots, s$. Denote the resulting manifold by $\left(g, n-s ; \frac{b_{1}}{a_{1}}, \cdots, \frac{b_{s}}{a_{s}}\right)$ which has the Seifert fiber structure extended from the circle bundle structure of $J^{\prime}$. Each orientable Seifert fibered space with orientable base $F_{g, n-s}$ and with $\leq s$ exceptional fibers is obtained in such a way. If $J$ is closed, i.e. if $s=n$, then define the Euler number of the Seifert fibration by

$$
e(J)=\sum_{i=1}^{s} \frac{b_{i}}{a_{i}} \in \mathbb{Q}
$$

and the Euler characteristic of the orbifold $O(J)$ by

$$
\chi_{O(J)}=2-2 g-\sum_{i=1}^{s}\left(1-\frac{1}{a_{i}}\right) \in \mathbb{Q} .
$$

From [ [BG2] we know that a closed orientable 3 -manifold $J$ supports the $\widehat{\mathrm{SL}_{2}(\mathbb{R})}$ geometry, i.e. there is a discrete and faithful representation $\psi: \pi_{1} J \rightarrow \mathrm{IsoSL}_{2}(\mathbb{R})$, if and only if $J$ is Seifert fibered with non-zero Euler number $e(J)$ and negative Euler characteristic $\chi_{O(J)}$ of the base orbifold.

A properly $\pi_{1}$-injectively immersed subsurface $j: S \leftrightarrow J$ of finite type is said to be horizontal if it can be properly homotoped to be transverse to the fiber at any point. Otherwise it is said to be vertical, and in this case, it is an annulus or a torus fibering over a properly immersed path or loop in the base orbifold.

2.2. Volume of representations of closed manifolds. We recall three definitions of volume of representations.

2.2.1. Via developing maps. Given a semi-simple, connected Lie group $G$ and a closed oriented manifold $M^{n}$ of the same dimension as the contractible space $X^{n}=G / K$, where $K$ is a maximal compact subgroup of $G$, we can associate, to each representation $\rho: \pi_{1} M \rightarrow G$, a volume $\operatorname{vol}_{G}(M, \rho)$ in the following way. 
First fix a $G$-invariant Riemannian metric $g_{X}$ on $X$, and denote by $\omega_{X}$ the corresponding $G$-invariant volume form. Let $\widetilde{M}$ denote the universal covering of $M$. We think of the elements $\widetilde{x}$ of $\widetilde{M}$ as the homotopy classes of paths $\gamma:[0,1] \rightarrow M$ with $\gamma(0)=x_{0}$ which are acted by $\pi_{1}\left(M, x_{0}\right)$ by setting $[\sigma] . \widetilde{x}=[\sigma \cdot \gamma]$, where . denotes the paths composition.

A developing map $D_{\rho}: \widetilde{M} \rightarrow X$ associated to $\rho$ is a $\pi_{1} M$-equivariant map such that for any $x \in \widetilde{M}$ and $\alpha \in \pi_{1} M$, then

$$
D_{\rho}(\alpha . x)=\rho(\alpha)^{-1} D_{\rho}(x)
$$

where $\rho(\alpha)$ acts on $X$ as an isometry. Such a map does exist and can be constructed explicitly as in [BCG]: Fix a triangulation $\Delta_{M}$ of $M$. Then its lift is a triangulation $\Delta_{\widetilde{M}}$ of $\widetilde{M}$, which is $\pi_{1} M$-equivariant. Then fix a fundamental domain $\Omega$ of $M$ in $\widetilde{M}$ such that the zero skeleton $\Delta_{\widetilde{M}}^{0}$ misses the frontier of $\Omega$. Let $\left\{x_{1}, \ldots, x_{l}\right\}$ be the vertices of $\Delta_{\widetilde{M}}^{0}$ in $\Omega$, and let $\left\{y_{1}, \ldots, y_{l}\right\}$ be any $l$ points in $X$. We first set

$$
D_{\rho}\left(x_{i}\right)=y_{i}, i=1, \ldots, l .
$$

Next extend $D_{\rho}$ in an $\pi_{1} M$-equivariant way to $\Delta_{\widetilde{M}}^{0}$ : For any vertex $x$ in $\Delta_{\widetilde{M}}^{0}$, there is a unique vertex $x_{i}$ in $\Omega$ and $\alpha_{x} \in \pi_{1} M$ such that $\alpha_{x} \cdot x_{i}=x$, and we set $D_{\rho}(x)=$ $\rho\left(\alpha_{x}\right)^{-1} D_{\rho}\left(x_{i}\right)$. Finally we extend $D_{\rho}$ to edges, faces, etc., and $n$-simplices of $\Delta_{\widetilde{M}}$ by straightening the images to geodesics using the homogeneous metric on the contractible space $X$. This map is unique up to equivariant homotopy. Then $D_{\rho}^{*}\left(\omega_{X}\right)$ is a $\pi_{1} M$ invariant closed $n$-form on $\widetilde{M}$ and therefore can be thought of as a closed $n$-form on $M$. Thus define

$$
\operatorname{vol}_{G}(M, \rho)=\int_{M} D_{\rho}^{*}\left(\omega_{X}\right)=\sum_{i=1}^{s} \varepsilon_{i} \operatorname{vol}_{X}\left(D_{\rho}\left(\widetilde{\Delta}_{i}\right)\right)
$$

where $\left\{\Delta_{1}, \ldots, \Delta_{s}\right\}$ are the $n$-simplices of $\Delta_{M}, \widetilde{\Delta}_{i}$ is a lift of $\Delta_{i}$ and $\varepsilon_{i}= \pm 1$ depending on whether $D_{\rho} \mid \widetilde{\Delta}_{i}$ is preserving or reversing orientation.

2.2.2. Via continuous cohomology classes. Let $\mathfrak{g}$ and $\mathfrak{k}$ denote the Lie algebra of $G$ and $K$. Let $o=\{K\}$ be the base point of $X=G / K$ and for any $g_{1}, \ldots, g_{l} \in G$ denote by $\Delta\left(g_{1}, \ldots, g_{l}\right)$ the geodesic $l$-simplex of $X$ with vertices $\left\{o, g_{1}(o), \ldots, g_{l} \ldots g_{2} g_{1}(o)\right\}$. There is a natural homomorphism

$$
H^{*}(\mathfrak{g}, \mathfrak{k} ; \mathbb{R})=H^{*}(G \text {-invariant differential forms on } X) \rightarrow H_{\text {cont }}^{*}(G ; \mathbb{R})
$$

defined in [Du1] by $\eta \mapsto\left(\left(g_{1}, \ldots, g_{l}\right) \rightarrow \int_{\Delta\left(g_{1}, \ldots, g_{l}\right)} \eta\right)$ which turns out to be an isomorphism by the Van Est Theorem [V].

Recall that for each representation $\rho: \pi_{1} M \rightarrow G$ one can associate a flat bundle over $M$ with fiber $X$ and group $G$ constructed as follows: $\pi_{1} M$ acts diagonally on the product $\widetilde{M} \times X$ by the following formula

$$
\sigma \cdot(\widetilde{x}, g)=\left(\sigma . \widetilde{x}, \rho^{-1}(\sigma) g\right)
$$

and we can form the quotient $\widetilde{M} \times{ }_{\rho} X=(\widetilde{M} \times X) / \pi_{1} M$ which is the flat $X$-bundle over $M$ corresponding to $\rho$.

Then for each $G$-invariant closed form $\omega$ on $X, q^{*}(\omega)$ is a $\pi_{1}(M)$-invariant closed form on $\widetilde{M} \times X$, where $q: \widetilde{M} \times X \rightarrow X$ is the projection, which induces a form $\omega^{\prime}$ on $M \times{ }_{\rho} X$. Then $s^{*}\left(\omega^{\prime}\right)$ is a closed form on $M$, where $s: M \rightarrow M \times{ }_{\rho} X$ is a section 
(since $X$ is contractible, such a section exists all such sections are homotopic). Thus any representation $\rho: \pi_{1} M \rightarrow G$ leads to a natural homomorphism

$$
\rho^{*}: H_{\text {cont }}^{*}(G ; \mathbb{R})=H^{*}(G \text {-invariant differential forms on } X) \rightarrow H^{*}(M ; \mathbb{R})
$$

induced by $\rho^{*}(\omega)=s^{*} \omega^{\prime}$. The volume of $\rho$ is therefore defined by

$$
\operatorname{vol}_{G}(M, \rho)=\int_{M} \rho^{*}\left(\omega_{X}\right)
$$

The equivalence between the two definitions is immediate since the $\pi_{1} M$-equivariant map Id $\times \mathrm{D}_{\rho}: \widetilde{M} \rightarrow \widetilde{M} \times X$ descends to a section $M \rightarrow M \times \times_{\rho} X$.

2.2.3. Via transversely projective foliations. This definition only makes sense for the Seifert volume. Let $\mathfrak{F}$ be a co-dimension one foliation on a closed smooth manifold $M$ determined by a 1 -form $\omega$. Then by the Froebenius Theorem one has $d \omega=\omega \wedge \delta$ for some 1 -form $\delta$. It was observed by Godbillon and Vey [GV] that the 3-form $\delta \wedge d \delta$ is closed and the class $[\delta \wedge d \delta] \in H^{3}(M, \mathbb{R})$ depends only on the foliation $\mathfrak{F}$ (and not on the chosen form $\omega$ ). This cohomology class is termed the Godbillon-Vey class of the foliation $\mathfrak{F}$ and denoted by $G V(\mathfrak{F})$.

Proposition 2.4 ([BG1], Proposition 1]). Suppose $\mathfrak{F}$ is a horizontal flat foliation on a circle bundle $\mathbf{S}^{1} \rightarrow E \rightarrow M$ with structural group $\mathrm{PSL}_{2}(\mathbb{R})$. Then

$$
\int_{\mathbf{S}^{1}} G V(\mathfrak{F})=4 \pi^{2} \widetilde{e}(E),
$$

where $\int_{\mathbf{S}^{1}}: H^{3}(E) \rightarrow H^{2}(M)$ denotes the integration along the fiber and $\widetilde{e}$ denotes the Euler class of the bundle.

Let $M$ be a closed orientable 3-manifold and $\phi: \pi_{1} M \rightarrow \mathrm{PSL}_{2}(\mathbb{R})$ be a representation with zero Euler class. Since PSL $2(\mathbb{R})$ acts on $\mathbf{S}^{1}$ then one can consider the corresponding flat circle bundle $M \times{ }_{\phi} \mathbf{S}^{1}$ over $M$ and the associated horizontal $\left(\mathrm{PSL}_{2}(\mathbb{R}), \mathbf{S}^{1}\right)$-foliation $\mathfrak{F}_{\phi}$. Since the Euler class of $\phi$ is zero we can choose a section $\delta$ of $M \times_{\phi} \mathbf{S}^{1} \rightarrow M$. Brooks and Goldman showed that $\delta^{*} G V\left(\mathfrak{F}_{\phi}\right)$ only depends on $\phi$ (and not on a chosen section $\delta$ ) [BG1, Lemma 2]. Then they defined the Godbillon-Vey invariant of $\phi$ by setting

$$
G V(\phi)=\int_{M} \delta^{*} G V\left(\mathfrak{F}_{\phi}\right)
$$

For a given representation $\phi: \pi_{1} M \rightarrow \mathrm{PSL}_{2}(\mathbb{R}), \phi$ lifts to $\widetilde{\phi}: \pi_{1} M \rightarrow \widetilde{\mathrm{SL}_{2}(\mathbb{R})}$ if and only if $\widetilde{e}(\phi)=0$ in $H^{2}(M, \mathbb{Z})$. The following fact has been verified in [BG1].

Proposition 2.5. Let $M$ be a closed oriented 3-manifold, let $\phi: \pi_{1} M \rightarrow \mathrm{PSL}_{2}(\mathbb{R})$ be a representation with zero Euler class and fix a lift $\tilde{\phi}: \pi_{1} M \rightarrow \widetilde{\mathrm{SL}_{2}(\mathbb{R})}$ of $\phi$. Then

$$
G V(\phi)=\operatorname{vol}_{\mathrm{SL}_{2}(\mathbb{R})}(M, \widetilde{\phi}),
$$

where $\widetilde{\mathrm{SL}_{2}(\mathbb{R})}$ is viewed as a semi-simple Lie group acting on itself by multiplication with corresponding homogeneous space $\widetilde{\mathrm{SL}_{2}(\mathbb{R})}$. 


\section{CHERN-SIMONS THEORY AND ADDITIVITY PRINCIPLE}

In this section, we consider volume of representations from the perspective of ChernSimons theory. In particular, we prove the additivity principle (Theorem 3.5).

Throughout this section we refer to $[\mathrm{CS}]$ and $[\mathrm{KN}]$. In this part, all the objects we deal with are smooth. Let $\pi: P \rightarrow M$ denote a principal $G$-bundle over a closed manifold $M$. We suppose that $G$ is a Lie group acting on the right on $P$ and we denote by $R_{g}$ the right action

$$
P \ni x \mapsto x . g \in P
$$

where $g$ in an element of $G$. Denote by $\mathfrak{g}$ the Lie algebra of $G$. Let $V P$ be the vertical subbundle of $T P$.

Let $P_{1}$ and $P_{2}$ denote two principal $G_{1}$, respectively, $G_{2}$-bundles over manifolds $M_{1}$ respectively, $M_{2}$. Following the formalism in [KN, p. 53] a homomorphism of principal bundles consists of a map $f: P_{1} \rightarrow P_{2}$ as well as a homomorphism $f^{\prime}: G_{1} \rightarrow G_{2}$ such that $f(x . g)=f(x) \cdot f^{\prime}(g)$, where $x \in P_{1}$ and $g \in G_{1}$. We say that a bundle homomorphism induces the identity in the structural group if $G_{1}=G_{2}=G$ and $f^{\prime}$ is the identity map.

3.1. Connections on principal bundles. We denote by $\Omega^{k}(P ; \mathfrak{g})$ the set of differential $k$-forms taking values in $\mathfrak{g}$. We define the exterior product of $\omega_{1} \in \Omega^{k}(P ; \mathfrak{g})$ by $\omega_{2} \in$ $\Omega^{l}(P ; \mathfrak{g})$ as an element $\omega_{1} \wedge \omega_{2}$ of $\Omega^{k+l}(P ; \mathfrak{g} \otimes \mathfrak{g})$ by setting

$$
\begin{gathered}
\omega_{1} \wedge \omega_{2}\left(X_{1}, \ldots, X_{k+l}\right)= \\
\frac{1}{(k+l) !} \sum_{\sigma \in \mathfrak{S}_{k+l}} \operatorname{sign}(\sigma) \omega_{1}\left(X_{\sigma(1)}, \ldots, X_{\sigma(k)}\right) \otimes \omega_{2}\left(X_{\sigma(k+1)}, \ldots, X_{\sigma(l)}\right) .
\end{gathered}
$$

The Lie bracket $[.,$.$] in \mathfrak{g}$ induces a map $\Omega^{k+l}(P ; \mathfrak{g} \otimes \mathfrak{g}) \rightarrow \Omega^{k+l}(P ; \mathfrak{g})$ and we denote $\left[\omega_{1}, \omega_{2}\right]$ the image of $\omega_{1} \wedge \omega_{2}$ under this maps. Explicitly we get:

$$
\begin{gathered}
{\left[\omega_{1}, \omega_{2}\right]\left(X_{1}, \ldots, X_{k+l}\right)=} \\
\frac{1}{(k+l) !} \sum_{\sigma \in \mathfrak{S}_{k+l}} \operatorname{sign}(\sigma)\left[\omega_{1}\left(X_{\sigma(1)}, \ldots, X_{\sigma(k)}\right), \omega_{2}\left(X_{\sigma(k+1)}, \ldots, X_{\sigma(l)}\right)\right] .
\end{gathered}
$$

The differential $d: \Omega^{k}(P ; \mathfrak{g}) \rightarrow \Omega^{k+1}(P ; \mathfrak{g})$ is defined by the Cartan formula

$$
\begin{gathered}
d \omega\left(X_{1}, \ldots, X_{k+1}\right)=\frac{1}{k+1} \sum_{i=1}^{k+1} X_{i} \cdot \omega\left(X_{1}, \ldots, \widehat{X}_{i}, \ldots, X_{k+1}\right)+ \\
\frac{1}{k+1} \sum_{i<j}(-1)^{i+j} \omega\left(\left[X_{i}, X_{j}\right], X_{1}, \ldots, \widehat{X}_{i}, \ldots, \widehat{X}_{j}, \ldots, X_{p+1}\right) .
\end{gathered}
$$

The derivative at the identity 1 of $G$ of the map

$$
G \ni g \mapsto x . g \in P
$$

induces an isomorphism $\nu_{x}: \mathfrak{g} \rightarrow V_{x} P \subset T_{x} P$ and we get the exact sequence

$$
0 \rightarrow \mathfrak{g} \stackrel{\nu_{x}}{\rightarrow} T_{x} P \stackrel{d \pi_{x}}{\rightarrow} T_{\pi(x)} M \rightarrow 0 .
$$

A horizontal subbundle $H P$ of $T P$ is a smooth distribution such that $T_{x} P=V_{x} P \oplus H_{x} P$ for any $x \in P$ that is $G$ equivariant: $H_{x . g}=d R_{g}(x) H_{x}$. This is given equivalently by the kernel of an element $\omega \in \Omega^{1}(P ; \mathfrak{g})$ such that for any $x \in P$

(1) $\omega_{x} \circ \nu_{x}=\operatorname{Id}_{\mathfrak{g}}$ and

(2) $R_{g}^{*} \omega=\operatorname{Ad}_{g^{-1}}(\omega)$. 
An element of $\Omega^{1}(P ; \mathfrak{g})$ satisfying (1) and (2) is termed a connection of $P$. Denote by $\mathcal{A}(P)$ the space of all conections on $P$. This space is naturally acted on by the gauge group denoted by $\mathcal{G}_{P}$ consisting of the $G$-equivariant bundle automorphisms of $P$.

The basic example is the group $G$ itself, viewed as a trivial bundle over a point or more generally the trivialized bundle $M \times G$ with the so-called Maurer-Cartan connection $\omega_{\text {M.C. }}=d\left(L_{g^{-1}} \circ \pi_{2}\right)$, where $L_{g}$ denotes the left translation in $G$ and $\pi_{2}$ the projection of $P$ onto $G$. This connection satisfies the Maurer-Cartan equation, namely

$$
d \omega_{\text {M.C. }}=-\frac{1}{2}\left[\omega_{\text {M.C. }}, \omega_{\text {M.C. }}\right] \text {. }
$$

Let us make a concrete computation for $G$. Let $X_{1}, \ldots, X_{n}$ be a basis of $\mathfrak{g}$. Since $\mathfrak{g}$ can be thought of as the space of left invariant vector fields in $G$, its dual $\mathfrak{g}^{*}$ is the space of left invariant differential 1-forms on $G$. Let $\theta^{1}, \ldots, \theta^{n}$ denote the dual basis of $\mathfrak{g}^{*}$. Then

$$
\omega_{\text {M.C. }}=\theta^{1} \otimes X_{1}+\ldots+\theta^{n} \otimes X_{n} .
$$

Let us write the constants structure of $\mathfrak{g}$ which are given by the formula

$$
\left[X_{j}, X_{k}\right]=\sum_{i} c_{j k}^{i} X_{i}
$$

Thus by the Maurer-Cartan equation we get the equalities

$$
d \theta^{i}=-\frac{1}{2} \sum_{j, k} c_{j, k}^{i} \theta^{j} \wedge \theta^{k}
$$

In general, for a given connection $\omega$ in a bundle $P$, the element

$$
F^{\omega}=d \omega+\frac{1}{2}[\omega, \omega]
$$

is the curvature of $\omega$ lying in $\Omega^{2}(P ; \mathfrak{g})$ and measuring the integrability of the corresponding horizontal distribution. When $F^{\omega}=0$ we say that the connection is flat. Denote by $\mathcal{F} \mathcal{A}(P)$ the subset of $\mathcal{A}(P)$ which consists of flat connections on $P$. This space is preserved by the gauge group action.

We recall the following basic fact that will be used very often in this paper. To each flat connection $\omega$ one can associate a representation $\rho: \pi_{1} M \rightarrow G$ by lifting the loops of $M$ in the leaves of the horizontal foliation given by integrating the distribution ker $\omega$.

On the other hand $\omega$ can be recovered from $\rho$ by the following construction. The fundamental group of $M$ acts on the product $\widetilde{M} \times G$ by the formula $[\sigma] .([\gamma], g)=$ $\left([\sigma \cdot \gamma], \rho\left([\sigma]^{-1}\right) . g\right.$ ) and the quotient $\widetilde{M} \times{ }_{\rho} G$ under this $\pi_{1} M$-action is isomorphic to $P$ and the push forward of the vertical distribution of $\widetilde{M} \times G$ in $\widetilde{M} \times{ }_{\rho} G$ corresponds to $\omega$ in $P$. We get a natural map

$$
I_{P}: \mathcal{B}(P)=\mathcal{F} \mathcal{A}(P) / \mathcal{G}_{P} \hookrightarrow \mathcal{R}\left(\pi_{1} M, G\right) / \text { conjugation, }
$$

where $\mathcal{R}\left(\pi_{1} M, G\right)$ is the set of representations of $\pi_{1} M$ into $G$ acted by the conjugation in $G$.

3.2. Chern-Simons classes. Given a Lie group $G$, a polynomial of degree $l$ is a symmetric linear map $f: \otimes^{l} \mathfrak{g} \rightarrow \mathbb{K}$, where $\mathbb{K}$ denotes either the real or the complex numbers field. The group $G$ acts on $\mathfrak{g}$ by Ad and the polynomials invariant under this action are called the invariant polynomials of degree $l$ and are denoted by $I^{l}(G)$ with the convention $I^{0}(G)=\mathbb{K}$. Denote $I(G)$ the sum $\oplus_{l \in \mathbb{N}} I^{l}(G)$.

The Chern-Weil theory gives a correspondence $W_{P}$ from $I^{l}(G)$ to $H^{2 l}(M ; \mathbb{K})$ constructed in the following way. Choose a connection $\omega$ in $P$ then for any $l \geq 1$ a polynomial 
$f \in I^{l}(G)$ gives rise to a $2 l$-form $f\left(\wedge^{l} F^{\omega}\right)$ in $P$. It follows from the Chern-Weil Theory that $f\left(\wedge^{l} F^{\omega}\right)$ is closed and is the pull-back of a unique form on $M$ under $\pi: P \rightarrow M$ denoted by $\pi_{*} f\left(\wedge^{l} F^{\omega}\right)$. Then $W_{P}(f)$ is by definition the class of $\pi_{*} f\left(\wedge^{l} F^{\omega}\right)$ in $H^{2 l}(M)$. The Chern-Weil Theorem claims that $W_{P}(f)$ does not depend on the chosen connection $\omega$ and that $W_{P}$ is actually a homomorphism.

Let $E G$ denote the universal principal $G$-bundle and denote by $B G$ the classifying space of $G$. This means that any principal $G$-bundle $P \rightarrow M$ admits a bundle homomorphism $\xi: P \rightarrow E G$ descending to the classifying map, still denoted $\xi: M \rightarrow B G$, that is unique up to homotopy. There exists the universal Chern-Weil homomorphism $\widetilde{W}: I^{l}(G) \rightarrow H^{2 l}(B G)$ such that $\xi^{*} \widetilde{W}(f)=W_{P}(f)$.

The Chern-Simons invariants were derived from this construction by Chern and Simons who observed that $f\left(\wedge^{l} F^{\omega}\right)$, for $l \geq 1$, is actually exact in $P$ and a primitive is given explicitly in [CS] by

$$
T f(\omega)=l \int_{0}^{1} f\left(\omega \wedge\left(\wedge^{l-1} F^{t}\right)\right) d t
$$

where $F^{t}=t F^{\omega}+\frac{1}{2}\left(t^{2}-t\right)[\omega, \omega]$. The form $T f(\omega)$ is closed when $M$ is of dimension $2 l-1$. For instance when $l=2$ and $M$ is a 3-manifold, plugging $F^{t}$ and (3.2) into (3.3) we get a closed 3 -form on $P$, namely

$$
T f(\omega)=f\left(F^{\omega} \wedge \omega\right)-\frac{1}{6} f(\omega \wedge[\omega, \omega])=f(d \omega \wedge \omega)+\frac{1}{3} f(\omega \wedge[\omega, \omega])
$$

Considering $G$ as a principal bundle over the point this yields to

$$
T f\left(\omega_{\text {M.C. }}\right)=-\frac{1}{6} f\left(\omega_{\text {M.C. }} \wedge\left[\omega_{\text {M.C. }}, \omega_{\text {M.C. }}\right]\right) .
$$

The $(2 l-1)$-form $T f\left(\omega_{\text {M.C. }}\right)$ is closed, bi-invariant and defines a class in $H^{2 l-1}(G ; \mathbb{R})$. Let us denote by

$$
I_{0}(G)=\left\{f \in I(G), T f\left(\omega_{\text {M.C. }}\right) \in H^{2 l-1}(G ; \mathbb{Z})\right\} .
$$

The elements of $I_{0}(G)$ are termed integral polynomials. If $f \in I_{0}(G)$ then there is a well defined functional

$$
\mathfrak{c s}_{M}^{*}: \mathcal{A}_{M \times G} \rightarrow \mathbb{K} / \mathbb{Z}
$$

defined as follows: since $P=M \times G$ is a trivial(ized) we can consider, for any section $\delta$, the Chern-Simons invariant

$$
\mathfrak{c s}_{M}(\omega, \delta)=\int_{M} \delta^{*} T f(\omega)
$$

Since $f$ is an integral polynomial, the element $\mathfrak{c s}_{M}(\omega, \delta)$ is well defined modulo $\mathbb{Z}$ when the section changes. Then define $\mathfrak{c s}_{M}^{*}(\omega)$ to be the class of $\mathfrak{c s}_{M}(\omega, \delta)$ in $\mathbb{K} / \mathbb{Z}$.

The fundamental classical examples are $G=\mathrm{SU}(2 ; \mathbb{C})$ and $G=\mathrm{SO}(3 ; \mathbb{R})$.

The Chern-Simons classes for the group $\mathrm{SU}(2 ; \mathbb{C})$ are based on the second Chern class $f=C_{2} \in I_{0}^{2}(\mathrm{SL}(2 ; \mathbb{C}))$. We recall that the Chern classes, denoted by $C_{1}, C_{2}$ for $\mathrm{SU}(2 ; \mathbb{C})$, are the complex valued invariant polynomials such that

$$
\operatorname{det}\left(\lambda \cdot I_{2}-\frac{1}{2 i \pi} A\right)=\lambda^{2}+C_{1}(A) \lambda+C_{2}(A \otimes A),
$$


when $A \in \mathfrak{s l}_{2}(\mathbb{C})$. Thus after developing this equality we get

$$
C_{2}(A \otimes A)=\frac{1}{8 \pi^{2}} \operatorname{tr}\left(A^{2}\right)
$$

so that we get the usual formula (using (3.4))

$$
\begin{gathered}
T C_{2}(\omega)=\frac{1}{8 \pi^{2}} \operatorname{Tr}\left(F^{\omega} \wedge \omega-\frac{1}{6} \omega \wedge[\omega, \omega]\right) \\
=\frac{1}{8 \pi^{2}} \operatorname{Tr}\left(d \omega \wedge \omega+\frac{1}{3} \omega \wedge[\omega, \omega]\right)
\end{gathered}
$$

The Chern-Simons classes of the special orthogonal group $G=\mathrm{SO}(3 ; \mathbb{R})$ are based on the first Pontrjagin class $f=P_{1} \in I_{0}^{2}(\mathrm{SO}(3 ; \mathbb{R}))$ that is a the real valued invariant polynomial such that

$$
\operatorname{det}\left(\lambda \cdot I_{3}-\frac{1}{2 \pi} A\right)=\lambda^{3}+P_{1}(A \otimes A) \lambda,
$$

when $A \in \mathfrak{s o}_{3}(\mathbb{R})$. Thus after developing this equality we get

$$
P_{1}(A \otimes A)=-\frac{1}{8 \pi^{2}} \operatorname{tr}\left(A^{2}\right) .
$$

Example 3.1. When $M$ is an oriented Riemaniann closed $n$-manifold one can consider its associated $\mathrm{SO}(n ; \mathbb{R})$-bundle $\mathrm{SO}(M)$ which consists of the positive orthonormal unit frames endowed with the Levi Civita connection. When $M$ is of dimension 3 it is well known that its is parallelizable so that there exist sections $\delta$ of $\mathrm{SO}(M) \rightarrow M$. Therefore one can consider the Chern-Simons invariant of the Levi Civita connection on $M$ that will be denoted by $\mathfrak{c s}_{\text {L.C. }}(M, \delta)$.

A natural question arises in the following situation. There is an epimorphism $\pi_{2}: \mathrm{SU}(2 ; \mathbb{C}) \rightarrow \mathrm{SO}(3 ; \mathbb{R})$ that is the 2 -fold universal covering. Thus any connection $\omega$ on the trivialized $\mathrm{SU}(2 ; \mathbb{C})$-bundle over $M$ induces a connection $\omega^{\prime}$ on the corresponding $\mathrm{SO}(3 ; \mathbb{R})$-bundle over $M$. How can we compute $T P_{1}\left(\omega^{\prime}\right)$ from $T C_{2}(\omega)$ ? The answer is given in [KK] pp 543, end of Section 3] by recalling that $\pi_{2}$ induces a homomorphism between the corresponding classifying spaces

$$
\pi_{2}^{*}: H^{4}(B \mathrm{SO}(3 ; \mathbb{R})) \rightarrow H^{4}(B \mathrm{SU}(2 ; \mathbb{C})),
$$

such that

$$
\pi_{2}^{*} \widetilde{W}\left(P_{1}\right)=-4 \widetilde{W}\left(C_{2}\right) .
$$

Thus using the definition and the Chern-Weil universal homomorphism we get the equality

$$
\mathfrak{c s}_{M}\left(\omega^{\prime}, \delta^{\prime}\right)=-4 \mathfrak{c s}_{M}(\omega, \delta)
$$

where $\delta$ is a fixed section in the $\mathrm{SU}(2 ; \mathbb{C})$-bundle over $M$ and $\delta^{\prime}$ is the corresponding section in the $\mathrm{SO}(3 ; \mathbb{R})$-bundle over $M$. On the other hand since $G=\mathrm{SO}(3 ; \mathbb{R})$, respectively, $\mathrm{SU}(2 ; \mathbb{C})$, are the maximal compact subgroup of $\operatorname{PSL}(2 ; \mathbb{C})$, respectively, $\mathrm{SL}(2 ; \mathbb{C})$, whose quotients $\operatorname{PSL}(2 ; \mathbb{C}) / \mathrm{SO}(3 ; \mathbb{R})$, respectively, $\mathrm{SL}(2 ; \mathbb{C}) / \mathrm{SU}(2 ; \mathbb{C})$ are contractible then it follows from [Ho, Chapter 15, Theorem 3.1] and [Du2], Proposition 7.2, p. 98] that the natural inclusion gives rise to isomorphisms $H^{*}(B \operatorname{PSL}(2 ; \mathbb{C})) \rightarrow H^{*}(B S O(3 ; \mathbb{R}))$ and $H^{*}(B \mathrm{SL}(2 ; \mathbb{C})) \rightarrow H^{*}(B \mathrm{SU}(2 ; \mathbb{C}))$. We have the following commutative diagram

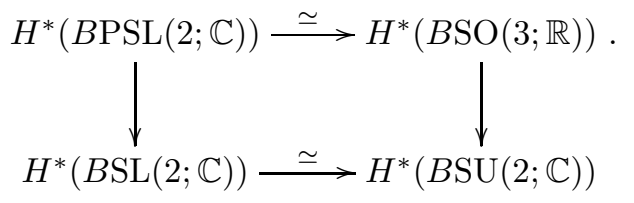


Hence we also get (fixing a trivialization, using (3.6), (3.7), (3.8))

$$
\begin{gathered}
\mathfrak{c s}_{M}\left(\omega^{\prime}, \delta^{\prime}\right)=-4 \mathfrak{c s}_{M}(\omega, \delta) \\
=-\frac{1}{2 \pi^{2}} \int_{M} \delta^{*} \operatorname{Tr}\left(F^{\omega} \wedge \omega-\frac{1}{6} \omega \wedge[\omega, \omega]\right)
\end{gathered}
$$

where $\delta$ is a fixed section in the $\mathrm{SL}(2 ; \mathbb{C})$-bundle over $M$ and $\delta^{\prime}$ is the corresponding section in the $\operatorname{PSL}(2 ; \mathbb{C})$-bundle over $M$.

3.3. Volume and Chern-Simons classes in Seifert geometry. In this section we check Proposition 1.9 (1) keeping the same notation as in the introduction. The proof is inspired from [BG2, p. 532] and we we will follow faithfully their presentation. If $G=$ $\mathrm{Iso}_{e}\left(\widetilde{\mathrm{SL}_{2}(\mathbb{R})}\right)$ then the matrices

$$
X=\left(\begin{array}{cc}
1 & 0 \\
0 & -1
\end{array}\right), Y=\left(\begin{array}{ll}
0 & 0 \\
1 & 0
\end{array}\right), \text { and } Z=\left(\begin{array}{ll}
0 & 1 \\
0 & 0
\end{array}\right),
$$

together with the generator $T$ of $\mathbb{R}$ form a basis of the Lie algebra $\mathfrak{g}$ of $G$. Setting $W=$ $Z-Y-T$ we get a new basis $\{X, Y, Z, W\}$ of $\mathfrak{g}$ with commutators relations

$$
\begin{gathered}
{[X, Y]=-2 Y,[X, Z]=2 Z} \\
{[Y, Z]=[Y, W]=[Z, W]=-X,[X, W]=2 Y+2 Z}
\end{gathered}
$$

which determine the coefficients in the Maurer-Cartan equations. Denote by $\varphi_{X}, \varphi_{Y}, \varphi_{Z}, \varphi_{W}$ the dual basis of $\mathfrak{g}^{*}$. The Maurer-Cartan form of $G$ is given by

$$
\omega_{\text {M.C. }}=\varphi_{X} \otimes X+\varphi_{Y} \otimes Y+\varphi_{Z} \otimes Z+\varphi_{W} \otimes W .
$$

Denote by $A$ a flat connection on $M \times{ }_{\rho} G$. By Section 3.1, if $\widetilde{M}$ denotes the universal covering and if $q: \widetilde{M} \times G \rightarrow G$ denotes the projection, then $A$ corresponds to the form $\overline{q^{*}\left(\omega_{\text {M.C. }}\right)}$, where $-: \widetilde{M} \times G \rightarrow \widetilde{M} \times{ }_{\rho} G$ denotes the push-forward which makes sense since $q^{*}\left(\omega_{\text {M.C. }}\right)$ is $\pi_{1} M$-invariant. The Chern-Simons class of the flat connection $A$ is $T \mathbf{R}(A)=\overline{q^{*} T \mathbf{R}\left(\omega_{\text {M.C. }}\right)}$. Using equations (3.1) and (3.10), we calculate

$$
\begin{array}{rlrl}
d \varphi_{X} & = & \varphi_{Y} \wedge \varphi_{Z}+\varphi_{Y} \wedge \varphi_{W}+\varphi_{Z} \wedge \varphi_{W} \\
d \varphi_{Y} & = & & 2 \varphi_{X} \wedge \varphi_{Y}-2 \varphi_{X} \wedge \varphi_{W} \\
d \varphi_{Z} & = & & -2 \varphi_{X} \wedge \varphi_{Z}-2 \varphi_{X} \wedge \varphi_{W} \\
d \varphi_{W} & = & 0
\end{array}
$$

Notice that those equations also imply that $2\left(\varphi_{X} \wedge \varphi_{Y}+\varphi_{X} \wedge \varphi_{Z}\right)=d\left(\varphi_{Y}-\varphi_{Z}\right)$ and therefore

$$
T \mathbf{R}\left(\omega_{\text {M.C. }}\right)=\frac{2}{3} \varphi_{X} \wedge \varphi_{Y} \wedge \varphi_{Z}+\frac{1}{3} d\left(\varphi_{Y} \wedge \varphi_{W}-\varphi_{Z} \wedge \varphi_{W}\right)
$$


The end of the proof follows from the commutativity of the diagram below and from the Stokes formula, since $\varphi_{X} \wedge \varphi_{Y} \wedge \varphi_{Z}$ represents the volume form on $X=\widetilde{\mathrm{SL}_{2}(\mathbb{R})}$.

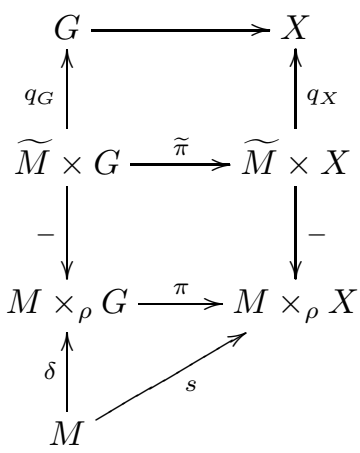

This completes the proof of Proposition 1.9(1).

3.4. Volume and Chern-Simons classes in hyperbolic geometry. We now check Proposition 1.9 (2). The following construction is largely inspired from [KK. pp. 553-556], using a formula established by Yoshida in [Yo].

Denote by $p: \operatorname{PSL}(2 ; \mathbb{C}) \simeq \mathrm{Iso}_{+} \mathbf{H}^{3} \rightarrow \mathbf{H}^{3}$ the natural projection. For short denote $\operatorname{PSL}(2 ; \mathbb{C})$ by $G$. For each representation $\rho: \pi_{1} M \rightarrow G$ admitting a lift into $\operatorname{SL}(2 ; \mathbb{C})$, we have the (trivial) principal bundle $M \times{ }_{\rho} G$ and the associated bundle $M \times{ }_{\rho} \mathbf{H}^{3}$. Denote by $A$ the flat connection over $M$ corresponding to $\rho$ and $\omega_{\mathbf{H}^{3}}$ the $G$-invariant volume form on $\mathbf{H}^{3}$ corresponding to the hyperbolic metric.

The matrices $X=\left(\begin{array}{cc}1 & 0 \\ 0 & -1\end{array}\right), Y=\left(\begin{array}{ll}0 & 0 \\ 1 & 0\end{array}\right), Z=\left(\begin{array}{ll}0 & 1 \\ 0 & 0\end{array}\right)$ form a basis of the Lie algebra $\mathfrak{s l}(2 ; \mathbb{C})$ with commutators relations

$$
[X, Y]=-2 Y,[X, Z]=2 Z,[Y, Z]=-X .
$$

Denote by $\varphi_{X}, \varphi_{Y}, \varphi_{Z}$ the dual basis of $\mathfrak{s l}^{*}(2 ; \mathbb{C})$. The Maurer-Cartan form of $G$ is

$$
\omega_{\mathrm{M} . \mathrm{C} .}=\varphi_{X} \otimes X+\varphi_{Y} \otimes Y+\varphi_{Z} \otimes Z,
$$

and

$$
T P_{1}\left(\omega_{\text {M.C. }}\right)=\frac{1}{\pi^{2}} \varphi_{X} \wedge \varphi_{Y} \wedge \varphi_{Z} .
$$

By the formula of Yoshida in [Yo] we know that

$$
i T P_{1}\left(\omega_{\text {M.C. }}\right)=\frac{1}{\pi^{2}} p^{*} \omega_{\mathbf{H}^{3}}+i \mathfrak{c s}_{\text {L.C. }}\left(\mathbf{H}^{3}\right)+d \gamma,
$$

where $p^{*} \omega_{\mathbf{H}^{3}}$ is the pull-back of $\omega_{\mathbf{H}^{3}}$ under the projection $p: \operatorname{PSL}(2 ; \mathbb{C}) \rightarrow \mathbf{H}^{3}$, $\mathfrak{c}_{\text {L.C. }}\left(\mathbf{H}^{3}\right)$ is the Chern-Simons 3-form of the Levi Civita connection over $\mathbf{H}^{3}$ (see Example 3.1) with the hyperbolic metric in its $\operatorname{SO}(3)$-frame bundle $\operatorname{PSL}(2 ; \mathbb{C})$ and $d \gamma$ is an 
exact real form. Consider the following commutative diagram

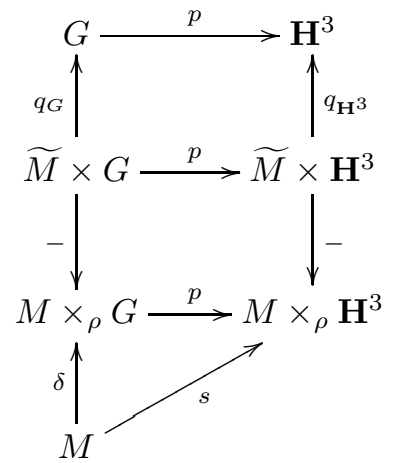

Notice that the sections in the bottom triangle are obtained as follows. Since $M$ is a 3manifold then it follows from the obstruction theory that any principal bundle with simply connected group is trivial. Since $\rho: \pi_{1} M \rightarrow G$ admits a lift into $\operatorname{SL}(2 ; \mathbb{C}) M \times{ }_{\rho} G$ is trivial. Denote by $\delta$ a section of $M \times{ }_{\rho} G \rightarrow M$. It induces, by $p \circ \delta=s$, a section of $M \times{ }_{\rho} \mathbf{H}^{3} \rightarrow M$.

Since all the maps are clear from the context, in the sequel, we will drop the index in the projections $q_{G}$ and $q_{\mathbf{H}^{3}}$ and we denote them just by $q$. Now the 3-form $\omega_{\mathbf{H}^{3}}$ induces a 3 -form $\overline{q^{*} \omega_{\mathbf{H}^{3}}}$ on $M \times{ }_{\rho} \mathbf{H}^{3}$ and

$$
i \overline{q^{*} T P_{1}\left(\omega_{\text {M.C. }}\right)}=\frac{1}{\pi^{2}} \overline{q^{*} p^{*} \omega_{\mathbf{H}^{3}}}+i \overline{q^{*} \mathfrak{c} \mathfrak{s}_{\text {L.C. }}\left(\mathbf{H}^{3}\right)}+\overline{q^{*} d \gamma}
$$

in $M \times_{\rho} G$, where the push-forward operation $\overline{q^{*}(.)}$ indeed makes sense since $T P_{1}\left(\omega_{\text {M.C. }}\right), p^{*} \omega_{\mathbf{H}^{3}}$ and $\mathfrak{c s}_{\text {L.C. }}\left(\mathbf{H}^{3}\right)$ are left invariant forms in $G$. Then

$$
i \mathfrak{c s}_{M}(A, \delta)=\frac{1}{\pi^{2}} \int_{M} \delta^{*} \overline{q^{*} p^{*} \omega_{\mathbf{H}^{3}}}+i \int_{M} \delta^{*} \overline{q^{*} \mathfrak{c s}_{\text {L.C. }}\left(\mathbf{H}^{3}\right)}+\int_{M} \delta^{*} \overline{q^{*} d \gamma}
$$

Since $\delta^{*} \overline{q^{*} p^{*} \omega_{\mathbf{H}^{3}}}=\delta^{*} p^{*} \overline{q^{*} \omega_{\mathbf{H}^{3}}}=s^{*} \overline{q^{*} \omega_{\mathbf{H}^{3}}}$ and $\int_{M} \delta^{*} \overline{q^{*} d \gamma}=0$ by the Stokes Formula, we have

$$
i \mathfrak{c s}_{M}(A, \delta)=\frac{1}{\pi^{2}} \int_{M} s^{*} \overline{q^{*} \omega_{\mathbf{H}^{3}}}+i \int_{M} \delta^{*} \overline{q^{*} \mathfrak{c s}_{\mathrm{L} . \mathrm{C} .}\left(\mathbf{H}^{3}\right)}=\frac{1}{\pi^{2}} \operatorname{vol}(M, \rho)+i \mathfrak{c s}\left(M_{\rho} ; \delta\right),
$$

where we denote $\int_{M} \delta^{*} \overline{q^{*} \mathfrak{c s}_{\text {L.C. }}\left(\mathbf{H}^{3}\right)}$ by $\mathfrak{c s}\left(M_{\rho} ; \delta\right)$. We get eventually

$$
\mathfrak{c s}_{M}(A, \delta)=\mathfrak{c s}\left(M_{\rho} ; \delta\right)-\frac{i}{\pi^{2}} \operatorname{vol}(M, \rho)
$$

This completes the proof of Proposition 1.9(2).

3.5. Normal form near toral boundary of 3-manifolds. In this part we recall the machinery developed in $[\mathrm{KK}]$. Let $M$ be a compact oriented 3-manifold with toral boundary $\partial M$ endowed with a preferred basis $s, h$ of $H_{1}(\partial M ; \mathbb{Z})$ (this implies that for each component $T_{i}$ of $\partial M$, there is a basis $s_{i}, h_{i}$, but for simplicity, we omit the sub-index). Let $\rho: \pi_{1} M \rightarrow G$ be a representation where $G$ is either $\operatorname{PSL}(2 ; \mathbb{C})$ or $\widehat{\operatorname{SL}(2 ; \mathbb{R})}$. We consider the space of flat connections $\mathcal{F} \mathcal{A}(P)$ where $P$ is the trivialized bundle $M \times G$. For representations into $\widehat{\mathrm{SL}(2 ; \mathbb{R})}$ the corresponding principal bundles are always trivial whereas the representations $\rho$ into $\operatorname{PSL}(2 ; \mathbb{C})$ leading to a trivial bundle are precisely those who admit a lift $\bar{\rho}$ into $\operatorname{SL}(2 ; \mathbb{C})$. Moreover if follows from $[\overline{\mathrm{KK}}]$ and $[\mathrm{Kh}]$ that after a conjugation, the representation $\rho \mid \pi_{1} T$ can be put in normal form, which either hyperbolic, 
elliptic or parabolic. Since the parabolic form will not be used in the explicit way we only recall the definitions of those representations which are elliptic or hyperbolic in the $\operatorname{PSL}(2 ; \mathbb{C})$ case and elliptic in the $\widetilde{\mathrm{SL}(2 ; \mathbb{R})}$ case in the boundary of $M$. Then by $[\overline{\mathrm{KK}}]$, when $G=\operatorname{PSL}(2 ; \mathbb{C})$, we may assume, after conjugation, that there exist $\alpha, \beta \in \mathbb{C}$ such that

$$
\rho(s)=\left(\begin{array}{cc}
e^{2 i \pi \alpha} & 0 \\
0 & e^{-2 i \pi \alpha}
\end{array}\right) \text { and } \rho(h)=\left(\begin{array}{cc}
e^{2 i \pi \beta} & 0 \\
0 & e^{-2 i \pi \beta}
\end{array}\right) ;
$$

when $G=\widetilde{\mathrm{SL}(2 ; \mathbb{R})}$, after conjugation, we may assume that after projecting to $\operatorname{PSL}(2 ; \mathbb{R})$ there exist $\alpha, \beta \in \mathbb{R}$ such that

$$
s \mapsto\left(\begin{array}{cc}
\cos (2 \pi \alpha) & \sin (2 \pi \alpha) \\
-\sin (2 \pi \alpha) & \cos (2 \pi \alpha)
\end{array}\right) \quad \text { and } h \mapsto\left(\begin{array}{cc}
\cos (2 \pi \beta) & \sin (2 \pi \beta) \\
-\sin (2 \pi \beta) & \cos (2 \pi \beta)
\end{array}\right) .
$$

In either case, if $A$ denotes a connection on $P$ corresponding to $\rho$ then after a gauge transformation $g$ the connection $g * A$ is in normal form:

$$
g * A \mid T \times[0,1]=(i \alpha d x+i \beta d y) \otimes X .
$$

Let $M$ be a closed oriented 3-manifold and $\mathcal{T}$ be a union of finitely many tori cutting $M$ into $J_{1}, \ldots, J_{k}$. For each $T$ in $\mathcal{T}$, we endow a homology basis $\left(m_{T}, l_{T}\right)$ and a base point $x_{T}=m_{T} \cap l_{T}$; and for simplicity we assume $T$ shared by $J_{j}$ and $J_{l}, j \neq l$ (this condotion can be reached in a finite cover, see Lemma 2.1). With the setting above, we have the following cut and paste result according to the correspondence between connections in normal form and representations for manifolds with toral boundary due to Kirk and Klassen (the similar fact has been used in [DW]).

Lemma 3.2. Let $\rho_{i}: \pi_{1} J_{j} \rightarrow G$ be a elliptic/hyperbolic representation, where $G$ is either $\widehat{\mathrm{SL}(2 ; \mathbb{R})}$ or $\operatorname{PSL}(2 ; \mathbb{C}), i=1, \ldots, k$. If for each $T$ the induced representations $\rho_{j} \mid \pi_{1} T$ and $\rho_{l} \mid \pi_{1} T$ are conjugated in $G$, then there exists a global representations $\rho: \pi_{1} M \rightarrow G$ inducing $\rho_{i}$ over $J_{i}$ up to conjugacy, $i=1, \ldots, k$.

Proof. For each $T$ in $\mathcal{T}, \rho_{i}$ induces $\left.\rho_{i}\right|_{T}: \pi_{1}\left(T \times[-1,1], x_{T}\right) \rightarrow G$, where $i=j, l$, $T \times[-1,1]$ is a regular neighborhood of $T$ with $T \times[-1,0] \subset J_{j}$ and $T \times[0,1] \subset J_{l}$.

Denote by $A_{i}$ be a flat connection over $J_{i}$ corresponding to $\rho_{i}$. Then $A_{i} \mid T \times[-1,1]$ can be put into normal form after gauge-transformation. Specially, there exists $g_{i, T}: T \times$ $[-1,1] \rightarrow G, i=j, l$, such that $g_{i, T} * A_{i} \mid T \times[-1,1]=\left(i \alpha_{i, T} d x+i \beta_{i, T} d y\right) \otimes X$. By obstruction theory one can extend

$$
\coprod_{T \in \partial J_{i}} g_{i, T} \rightarrow G
$$

to gauge transformations $g_{i}: J_{i} \rightarrow G$.

Since for each torus $T,\left.\rho_{j}\right|_{T}$ and $\left.\rho_{l}\right|_{T}$ are conjugated, they have the same eigenvalues, therefore the connections $g_{j} * A_{j}$ and $g_{l} * A_{l}$ match on $T \times[-1,1]$. So their union define a flat and smooth connection $C$ over $M$ and therefore a representation $\rho$ of $\pi_{1} M$ into $G$.

We quote the following result stated in [KK, Lemma 3.3] with $G=\mathrm{SL}(2 ; \mathbb{C})$ and in [Kh, Theorem 4.2] with $G=\widetilde{\mathrm{SL}(2 ; \mathbb{R})}$, that will be used latter :

Proposition 3.3. Let $A$ and $B$ denote two flat connections in normal form over an oriented 3-manifold with toral boundary. If $A$ and $B$ are equal near the boundary and if they are gauge equivalent then

i) $\mathfrak{c s}_{M}(A, \delta)=\mathfrak{c s}_{M}(B, \delta)$ when $G=\widetilde{\mathrm{SL}(2 ; \mathbb{R})}$ and, 
ii) $\mathfrak{c s}_{M}(A, \delta)-\mathfrak{c s}_{M}(B, \delta) \in \mathbb{Z}$ when $G=\operatorname{PSL}(2 ; \mathbb{C})$.

The second statement follows from [KK, Lemma 3.3] using identity (3.9).

Remark 3.4. As a consequence of Proposition 3.3, if $A$ and $B$ are flat connections on a solid torus that are equal near the boundary then the associated representations are automatically conjugated so that the conclusion of the proposition applies.

3.6. Additivity principle. Fix a closed oriented 3-manifold $M$ and denote by $[M]$ its orientation class. Let $\mathcal{T}$ be a union of finitely many tori cutting $M$ into $J_{1}, \ldots, J_{k}$. For each $T \in \mathcal{T}$, suppose $T$ is shared by $J_{i}$ and $J_{j}$. Denote by $\left[J_{i}, \partial J_{i}\right]$ the induced orientations classes so that the induced orientations on $\partial J_{i}$ and $\partial J_{j}$ are opposite on $T$, and we have

$$
[M]=\sum_{i=1}^{k}\left[\left(J_{i}, \partial J_{i}\right)\right] .
$$

Fix a regular neighborhood $W(T)=[0,1] \times T$ such that $T=\{1 / 2\} \times T, J_{i} \cap W(T)=$ $[0,1 / 2] \times T$ and $J_{j} \cap W(T)=[1 / 2,1] \times T$. Let $A$ denote a flat connection over $M$. Applying the same arguments as in $[\mathrm{KK}]$ we may assume that $A \mid W(T)$ is in normal form. Then by linearity of the integration

$$
\mathfrak{c s}_{M}(A)=\sum_{i=1}^{k} \mathfrak{c s}_{J_{i}}\left(A \mid J_{i}\right)
$$

Denote by $V$ the solid torus with meridian $m$. Denote by $c$ a slope in $T$ and for each $T \in \mathcal{T}$, we perform a Dehn filling to $J_{i}$ identifying $c$ with $m$ and denote by $\widehat{M}_{i}=J_{i} \cup$ $\left(\cup_{c \subset \partial J_{i}} V_{c}\right)$ the resulting closed oriented manifold. Suppose each $A \mid M_{i}$ smoothly extend to flat connections over $\widehat{J}_{i}$ denoted by $\widehat{A}_{i}$ for $i \in\{1, \ldots, k\}$. This is to say that for any representation $\rho$ corresponding to $A$ then $[c] \in \operatorname{ker} \rho$. By the linearity we have

$$
\mathfrak{c s}_{\widehat{J}_{i}}\left(\widehat{A}_{i}\right)=\mathfrak{c s}_{J_{i}}\left(A \mid J_{i}\right)+\sum_{c \subset \partial J_{i}} \mathfrak{c s}_{V_{c}}\left(\widehat{A}_{i} \mid V_{c}\right) \quad(i) .
$$

Since the extensions from $J_{i}$ and $J_{j}$ over their own $V_{c}$, based on the normal form on $[0,1] \times T$, are the same on the $T$ direction but opposite on the $[0,1]$ direction, then using Proposition 3.3 and Remark 3.4 we have

$$
\mathfrak{c s}_{V_{c}}\left(\widehat{A}_{i} \mid V_{c}\right)+\mathfrak{c s}_{V_{c}}\left(\widehat{A}_{j} \mid V_{c}\right)=0
$$

Summing up $(i)$ from 1 to $k$, then apply $(*)$, we get

$$
\mathfrak{c s}_{M}(A)=\sum_{i=1}^{k} \mathfrak{c s}_{\widehat{J}_{i}}\left(A \mid \widehat{J}_{i}\right)
$$

Then applying Proposition 1.9 (1.2) and (1.3) in the introduction to the former equality we get the so-called additivity principle:

Theorem 3.5. Let $M$ is an oriented closed 3-manifold with JSJ tori $T_{1}, \cdots, T_{r}$ and JSJ pieces $J_{1}, \cdots, J_{k}$, and let $\zeta_{1}, \cdots, \zeta_{r}$ be slopes on $T_{1}, \cdots, T_{r}$, respectively.

Suppose that $G$ is either $\operatorname{Iso}_{e} \widehat{\mathrm{SL}_{2}(\mathbb{R})}$ or $\operatorname{PSL}(2 ; \mathbb{C})$, and that

$$
\rho: \pi_{1}(M) \rightarrow G
$$


is a representation vanishing on the slopes $\zeta_{i}$, and that $\widehat{\rho}_{i}: \pi_{1}\left(\widehat{J}_{i}\right) \rightarrow G$ are the induced representations, where $\widehat{J}_{i}$ is the Dehn filling of $J_{i}$ along slopes adjacent to its boundary, with the induced orientations. Then:

$$
\operatorname{vol}_{G}(M, \rho)=\operatorname{vol}_{G}\left(\widehat{J}_{1}, \widehat{\rho}_{1}\right)+\operatorname{vol}_{G}\left(\widehat{J}_{2}, \widehat{\rho}_{2}\right)+\ldots+\operatorname{vol}_{G}\left(\widehat{J}_{k}, \widehat{\rho}_{k}\right) .
$$

We end this section by a simple lemma which will be used later and which is based on computation already developed in [KK].

Lemma 3.6. Suppose that $G$ is either $\mathrm{Iso}_{e} \widetilde{\mathrm{SL}_{2}(\mathbb{R})}$ or $\operatorname{PSL}(2 ; \mathbb{C})$ and that $M$ is a closed oriented 3-manifold. If $\rho: \pi_{1} M \rightarrow G$ has image either infinite cyclic or finite, then $\operatorname{vol}_{G}(M, \rho)=0$.

Proof. Suppose first the image $\rho\left(\pi_{1} M\right)$ is a cyclic group generated by $g$. Since $G$ is path connected, there is a path connecting the unit $e$ and $g$ which provides a path of representation $\rho_{t}: \pi_{1} M \rightarrow G$ such that $\rho_{1}=\rho$ and $\rho_{0}$ is the trivial representation.

Consider the associated path of flat connections $A_{t}$. This path defines a connection $\mathbb{A}$ on the product $M \times[0,1]$ that is no longer flat but whose curvature $F^{\mathbb{A}}$ satisfies the equation $F^{\mathbb{A}} \wedge F^{\mathbb{A}}=0$ (this latter point follows from the fact that $F^{A_{t}}=0$ for any $t$ ). Hence it follows from the construction of the Chern-Simons invariant combined with the Stokes Formula that $\mathfrak{c s}_{M}\left(A_{1}\right)=\mathfrak{c s}_{M}\left(A_{0}\right)=0$. Hence $\operatorname{vol}_{G}(M, \rho)=0$ by Propsotion 1.9

Suppose then the image $\rho\left(\pi_{1} M\right)$ is a finite group $\Gamma$. Let $p: \tilde{M} \rightarrow M$ be the finite cover corresponding to the unit of $\Gamma$, then we have the induced trivial representation $\rho \circ p_{*}$ : $\pi_{1} \tilde{M} \rightarrow G$. Clearly $\operatorname{vol}_{G}\left(\tilde{M}, \rho \circ p_{*}\right)=0$. Then $\operatorname{by}_{\operatorname{vol}_{G}}\left(\tilde{M}, \rho \circ p_{*}\right)=|\Gamma| \operatorname{vol}_{G}(M, \rho)$ and therefore $\operatorname{vol}_{G}(M, \rho)=0$.

\section{PRZYTYCKI-WISE SUBSURFACES AND SEPARABILITY}

Sections 4 and 5 are prepared for the construction part of the proof of Theorem 1.6

This section is inspired by recent work of P. Przytycki and D. Wise on surface subgroups of mixed 3-manifolds [PW1, PW2, PW3]. We first review the merging trick which will be used repeatedly in the constructions of finite covers. Then we introduce the partial PW subsurfaces and the parallel cutting condition. We show that parallel-cutting partial PW subsurfaces with virtually prescribed boundary exist under very general conditions (Theorem 4.11, and that any parallel-cutting partial PW subsurface can virtually be arranged in nice position with respect to the JSJ tori (Theorem 4.12). These results are interesting on their own right from the perspective of geometric topology, and they should be extendable to certain more natural contexts. Besides techniques from Przytycki-Wise, the proofs essentially employ results of Wise [Wi] and Rubinstein-Wang [RW] as well.

For notational convenience and to avoid repetition we always, from now, consider mixed 3 -manifolds that contain no essential Klein bottles so that the JSJ decomposition coincide with the geometric decomposition. This causes no loss of generality as we are interested in virtual properties (Lemma2.1).

4.1. Merging finite covers. In the study of virtual properties of mixed 3-manifolds, we often need to construct a finite cover of a 3-manifold from given covers of geometric pieces. This is possible via a procedure called merging.

Definition 4.1. Let $M$ be a compact orientable irreducible 3-manifold with (possibly empty) incompressible toral boundary. For a positive integer $m$, we say that a finite cover $\tilde{M}$ is $J S J$ m-characteristic, if every elevation $\tilde{T}$ of a JSJ torus or of a boundary torus 
$T \subset \partial M$ is the $m$-characteristic cover of $T$, namely, which means that every slope of $\tilde{T}$ covers a slope of $T$ with degree $m$.

Proposition 4.2. Let $M$ be a compact orientable irreducible 3-manifold with (possibly empty) incompressible toral boundary. Suppose $J_{1}^{\prime}, \cdots, J_{s}^{\prime}$ are finite covers of all the JSJ pieces $J_{1}, \cdots, J_{s}$ of $M$, respectively. Then there is a positive integer $m_{0}$, satisfying the following. For any positive integral multiple $m$ of $m_{0}$, there is a regular finite cover $\tilde{M}$ of $M$, which is JSJ m-characteristic, such that any elevation $\tilde{J}_{i}$ of a JSJ piece $J_{i}$ is a cover of $J_{i}$ that factors through $J_{i}^{\prime}$.

Proof. First observe that if $M^{\prime}$ is a JSJ $m$-characteristic finite cover of $M$, then there is a further JSJ $m$-characteristic regular finite cover $\tilde{M}$ of $M$ that factors through $M^{\prime}$. To see this, we may choose base points and assume $M^{\prime}$ corresponds to a finite-index subgroup $\pi^{\prime}$ of the pointed fundamental group $\pi$ of $M$. Then the cover $\tilde{M}$ corresponding to the normal core $\tilde{\pi}=\cap_{g \in \pi}\left(g^{-1} \pi^{\prime} g\right)$ of $\pi^{\prime}$ is clearly a regular finite cover of $M$. To see that it is JSJ $m$-characteristic, note that for any torus subgroup $\tilde{P} \leq \tilde{\pi}$ that represents a JSJ or boundary torus $\tilde{T}$ of $\tilde{M}$, we have $\tilde{P}=P \cap \tilde{\pi}$ for some JSJ or boundary torus $T$ of $M$. Since $P \cap g^{-1} \pi^{\prime} g$ for any $g \in \pi$ is the $m$-characteristic subgroup of $P$, and the $m$-characteristic subgroup of $P$ is unique, denoted as $P_{m}$, then

$$
\tilde{P}=P \cap \tilde{\pi}=P \cap\left(\cap_{g \in \pi}\left(g^{-1} \pi^{\prime} g\right)\right)=\cap_{g \in \pi}\left(P \cap\left(g^{-1} \pi^{\prime} g\right)\right)=\cap_{g \in \pi} P_{m}=P_{m}
$$

is the $m$-characteristic subgroup of $P$ as well. In other words, any JSJ or boundary torus $\tilde{T}$ of $\tilde{M}$ is also an $m$-characteristic cover of a JSJ or boundary torus $T$ of $M$.

Furthermore, we may reduce the proof to the case when $M$ is either hyperbolic or Seifert fibered. In fact, assuming that we have proved that case, then applying the lemma to each $J_{i}$ allows us to take the positive integer $m_{0}\left(J_{i}\right)$ corresponding to each $J_{i}$. Let $m_{0}$ be the least common multiple of all $m_{0}\left(J_{i}\right)$. Then for any multiple $m$ of $J_{i}$, there is a further regular finite cover $\tilde{J}_{i}$ of $J_{i}^{\prime}$, which is JSJ $m$-characteristic over $J_{i}$. Now (see [Lu] 384-385) let $d_{i}$ be the degree of $\tilde{J}_{i}$ over $J_{i}$, and let $D$ be the least common multiple of all $d_{i}$. We take $\frac{D}{d_{i}}$ copies of each $\tilde{J}_{i}$. For any $T$ in $\partial J_{i}$, there will be exactly $\frac{D}{d_{i}} \cdot \frac{d_{i}}{m}=\frac{D}{m}$ elevations for each side of $T$, and they are all $m$-characteristic over $T$. Thus we may glue these copies naturally along boundary, and a connected component $\tilde{M}$ will be a finite cover of $M$ which is JSJ $m$-characteristic. The observation at the beginning of the proof allows us to pass to a further regular finite JSJ $m$-characteristic cover of $M$.

It remains to prove the result when $M$ is either hyperbolic or Seifert fibered. If $M$ is hyperbolic, the conclusion is implied by the omnipotence for cusped hyperbolic 3-manifolds due to Wise [Wi, Corollary 16.15] (cf. [PW3, Lemma 3.2]). Indeed, in this case, a cover $M^{\prime}$ is given and there is no JSJ torus inside $M$. Denote the boundary tori of $\partial M$ by $\{T\}$ and $\partial M^{\prime}$ by $\left\{T^{\prime}\right\}$. By [PW3, Lemma 3.2], there are finite covers $\left\{T^{\prime \prime}\right\}$ of $\left\{T^{\prime}\right\}$ such that for any further finite cover $\{\tilde{T}\}$ of $\left\{T^{\prime \prime}\right\}$ there is a finite cover $\tilde{M}$ of $M^{\prime}$ so that the restriction on the boundary is the cover $\{\tilde{T}\}=\partial \tilde{M} \rightarrow\left\{T^{\prime}\right\}$. Therefore, we may pick a positive integer $m_{0}$ and $\{\tilde{T}\}$ above so that the composition $\{\tilde{T}\} \rightarrow\{T\}$ is $m_{0}$-characteristic, and then the composition $\tilde{M} \rightarrow M$ is a desired cover. If $M$ is Seifert fibered, the conclusion can be seen directly. Indeed, in this case, suppose $S^{1} \rightarrow M \rightarrow \mathcal{O}$ is the Seifert fibration over the base orbifold $\mathcal{O}$. Let $m_{0}$ be the maximal order of torsion elements of $H_{1}(\mathcal{O} ; \mathbb{Z})$. Since $M$ contains no essential Klein bottles, $H_{1}(M ; \mathbb{Z}) \cong H_{1}\left(S^{1} ; \mathbb{Z}\right) \oplus H_{1}(\mathcal{O} ; \mathbb{Z})$. Thus, the cover $\tilde{M}_{0}$ corresponding to the kernel of $\pi_{1}(M) \rightarrow H_{1}\left(M ; \mathbb{Z}_{m_{0}}\right)$ is a regular finite cover of $M$ that is $m_{0}$-characteristic on the boundary. For any positive multiple $m$ of $m_{0}$, we may take $\tilde{M}$ to be the cover corresponding to the kernel of $\pi_{1}\left(\tilde{M}_{0}\right) \rightarrow H_{1}\left(M ; \mathbb{Z}_{m / m_{0}}\right)$. 
Note that $\tilde{M}_{0}$ is homeomorphic to a product of $S^{1}$ with an orientable compact surface, $\tilde{M}$ is a regular finite cover of $M$ which is $m$-characterisitc on the boundary. This means $m_{0}$ is as desired.

Remark 4.3. In the proof of Proposition 4.2, if the cover $\tilde{M}$ is chosen to be corresponding to the subgroup $\tilde{\pi}=\cap_{\alpha \in \operatorname{Aut}\left(\pi^{\prime}\right)} \alpha\left(\pi^{\prime}\right)$, where $\operatorname{Aut}\left(\pi^{\prime}\right)$ is the automorphism group of $\pi^{\prime}$, it will be a characteristic finite cover of $M$, and similarly we can verify that this cover is JSJ $m$-characteristic.

Definition 4.4 (Cf. [PW3, Definition 3.3]). For a compact orientable irreducible 3manifold $M$ with (possibly empty) incompressible toral boundary, a semicover of $M$ with respect to the JSJ decomposition is a compact orientable irreducible 3-manifold $N$ together with an immersion $\mu: N \rightarrow M$, so that restricted to each component of $\partial N, \mu$ covers a JSJ torus of $M$.

Corollary 4.5 (Cf. [PW3, Proposition 3.4]). With the notations above, if $\mu: N \rightarrow M$ is a semicover, then there is a JSJ m-characteristic regular finite cover $\tilde{M}$ of $M$ in which a finite cover $\tilde{N}$ of $N$ is embedded as a chunk or a regular neighborhood of a JSJ torus, and the semicover $\tilde{N} \rightarrow M$ induced from $\mu$ is isotopic to the composition $\tilde{N} \hookrightarrow \tilde{M} \rightarrow M$.

Proof. This has essentially been proved in [PW3, Proposition 3.4], and we derive it from Proposition 4.2. For each JSJ piece $J_{i}$ of $M$, if $J_{i}$ is covered by a JSJ piece of $N$, let $J_{i}^{\prime}$ be a common finite cover of all JSJ pieces of $N$ that isotopically cover $J$, otherwise let $J_{i}^{\prime}=J_{i}$. By Proposition 4.2 we can obtain a JSJ $m$-characteristic regular finite cover $p^{\prime \prime}: M^{\prime \prime} \rightarrow$ $M$, such that any elevation $J_{i}^{\prime \prime}$ of a JSJ piece $J_{i}$ factors through $J_{i}^{\prime}$. Let $\mu^{\prime \prime}: N^{\prime \prime} \rightarrow M^{\prime \prime}$ be any elevation of $\mu$, where $N^{\prime \prime}$ covers $N$. Suppose $J_{*}^{\prime \prime}$ is an elevation of a JSJ-piece $J_{*}$ of $N$, then $J_{*}^{\prime \prime}$ covers a JSJ-piece $J_{i}^{\prime \prime}$ of $M^{\prime \prime}$ under $\mu^{\prime \prime}$, where $J_{i}^{\prime \prime}$ is an elevation of a JSJ-piece $J_{i}$ of $M$. By our construction we have $p_{*}^{\prime \prime}\left(\pi_{1}\left(J_{i}^{\prime \prime}\right)\right) \subset \mu\left(\pi_{1}\left(J_{*}\right)\right)$ and therefore $\mu_{*}^{\prime \prime} \mid: \pi_{1}\left(J_{*}^{\prime \prime}\right) \rightarrow \pi_{1}\left(J_{i}^{\prime \prime}\right)$ is surjective and $\mu^{\prime \prime} \mid: J_{*}^{\prime \prime} \rightarrow J_{i}^{\prime \prime}$ is a homeomorphism. That is $\mu^{\prime \prime}$ is an embedding restricted to each JSJ piece of $N^{\prime \prime}$.

Notice that the virtual embedding property is preserved after passing to a finite covering. Now either $N^{\prime \prime}$ is isotopic to a regular neighborhood of a JSJ torus of $M^{\prime \prime}$, or every JSJ piece of $N^{\prime \prime}$ is mapped to a unique JSJ piece of $M^{\prime \prime}$ by a homeomorphism. In the latter case, it follows that the induced map on the dual graph of the JSJ decompositions $\Lambda\left(N^{\prime \prime}\right) \rightarrow \Lambda\left(M^{\prime \prime}\right)$ is a canonical combinatorial local isometry, which is $\pi_{1}$-injective. Because $\pi_{1}\left(\Lambda\left(N^{\prime \prime}\right)\right)$ is a free group of finite rank, and hence is LERF, there is a finite cover $\tilde{\Lambda}$ of $\Lambda\left(M^{\prime \prime}\right)$, in which an elevation of $\Lambda\left(N^{\prime \prime}\right)$ is embedded as a complete subgraph. Therefore, we have a regular finite JSJ 1-characteristic covering $\tilde{M} \rightarrow M^{\prime \prime}$ so that any elevation $\tilde{\mu}: \tilde{N} \rightarrow \tilde{M}$ of $\mu^{\prime \prime}$ is an embedding. Therefore we have a JSJ $m$-characteristic covering $\tilde{M} \rightarrow M$ so that any elevation $\tilde{\mu}: \tilde{N} \rightarrow \tilde{M}$ of $\mu$ is an embedding. As we discussed at the beginning proof of Proposition 4.2 by passing to a further finite covering, we may assume that $\tilde{M} \rightarrow M$ is regular.

\subsection{PW subsurfaces and partial PW subsurfaces.}

Definition 4.6. Let $M$ be a closed orientable irreducible mixed 3-manifold containing no essential Klein bottles. A Przytycki-Wise subsurface of $M$, or simply a PW subsurface, is an immersed closed orientable surface

$$
j: S \rightarrow M
$$

in minimal general position with respect to the JSJ decomposition, satisfying the following: 
- $j$ is $\pi_{1}$-injective;

- for each maximal graph-manifold chunk $Q$ of $M$, each component of $j^{-1}(Q)$ is virtually embedded in $Q$; and

- for each hyperbolic piece $J$ of $M$, each component of $j^{-1}(J)$ is geometrically finite in $J$.

We may also regard any JSJ torus as a basic PW subsurface.

In our discussion, it will usually be convenient to regard the unpointed fundamental group $\pi_{1}(M)$ of a 3-manifold $M$ as the group of deck transformations on its universal cover $\widehat{M}$. Then, by a PW surface subgroup, we mean the stabilizer of an elevation in $\widehat{M}$ of a PW subsurface, which depends on the choice of the elevation.

Recall that a subset $W$ of a group $G$ is said to be separable if it is closed in the profinite topology. More precisely, this means that for any $h \in G$ not contained in $W$, there is a finite quotient $\bar{G}$ in which $\bar{h} \notin \bar{W}$.

The following lemma is a consequence of [PW2, Strong separation property] and [HW, Corollary 9.20].

Lemma 4.7. Let $M$ be a closed orientable irreducible mixed 3-manifold $M$. Then every $P W$ surface subgroup of $\pi_{1}(M)$ is separable.

We introduce the notion of partial PW subsurfaces.

Definition 4.8. Let $M$ be an orientable closed irreducible mixed 3-manifold. A partial $P W$ subsurface is a triple $(S, R, j)$ satisfying the following:

- $j: S \uparrow M$ is a PW subsurface of $M$;

- $R \subset S$ is a connected compact essential subsurface; and

- every component of $\partial R$ is immersed into a JSJ torus under $j$.

We often ambiguously say that $j: R \leftrightarrow M$ is a partial PW subsurface, with the triple $(S, R, j)$ implicitly assumed. The boundary of the partial PW subsurface is the boundary of $R$.

Definition 4.9. Let $M$ be an orientable closed irreducible mixed 3-manifold containing no essential Klein bottles. Let $J_{0}$ be a JSJ piece, and $T_{0}$ be a JSJ torus adjacent to $J_{0}$, and $\zeta_{0}$ be a slope on $T_{0}$. A partial PW subsurface $j: R \rightarrow M$ is said to be virtually bounded by $\zeta_{0}$ outside $J_{0}$, if the boundary $\partial R$ of $R$ is nonempty, covering $\zeta_{0}$ under $j$, and if the interior $\stackrel{\circ}{R}$ of $R$ misses $J_{0}$ under $j$. In this case, the carrier chunk $X(R) \subset M$ of $R$ is the unique minimal chunk that contains $R$, and the carrier boundary of $X(R)$ is the component $T_{0} \subset \partial X(R)$.

Definition 4.10. We say that a partial PW subsurface $j: R \rightarrow M$ is parallel cutting if for every JSJ torus $T \subset M$, all components of $j^{-1}(T)$ in $R$ cover the same slope of $T$.

\subsection{Virtual existence of partial PW subsurfaces.}

Theorem 4.11. Let $M$ be an orientable closed irreducible mixed 3-manifold containing no essential Klein bottles. Let $\zeta_{0}$ be a slope on a JSJ torus $T_{0}$ adjacent to a JSJ piece $J_{0}$. Then for some finite cover $\tilde{M}$ of $M$ together with an elevation $\left(\tilde{J}_{0}, \tilde{T}_{0}, \tilde{\zeta}_{0}\right)$ of the triple $\left(J_{0}, T_{0}, \zeta_{0}\right)$, there exists a parallel-cutting partial $P W$ subsurface $\tilde{R} \rightarrow \tilde{M}$, bounded virtually by $\tilde{\zeta}_{0}$ outside $\tilde{J}_{0}$.

Proof. We need to strengthen some arguments in the work of Przytycki-Wise [PW1, PW2]. Below is an outline of the construction. Note that in Case 2 one needs a little extra argument. 
By Lemma 2.1, we may assume that every JSJ torus of $M$ is adjacent to two distinct pieces. We will rewrite $J_{0}$ as $J_{-}$, and write $J_{+} \subset M$ be the other JSJ piece adjacent to $T_{0}$. The discussion falls into three cases according to the types of the pieces $J_{ \pm}$.

Case 1. If $J_{ \pm}$are both hyperbolic, by [PW2, Proposition 3.11], we may construct two geometrically finite subsurfaces $R_{ \pm}, \pi_{1}$-injectively, properly immersed in $J_{ \pm}$, such that $\partial R_{ \pm}$are nonempty and cover $\zeta_{0}$. The merging trick allows us construct $S$. More precisely, we pass to a possibly disconnected finite cover $\tilde{R}_{ \pm}$of $R_{ \pm}$, so that they have the same number of boundary components, and such that all components of $\partial \tilde{R}_{ \pm}$cover $\zeta_{0}$ with the same unsigned degree, [PW2, Lemma 3.14]. Then $S$ can be obtained by arbitrarily matching up the boundary components and taking a connected component of the result. In this case, we do not need to pass to a further cover of $M$, so we take $\tilde{M}$ to be $M$, and $\left(\tilde{J}_{0}, \tilde{T}_{0}, \tilde{\zeta}_{0}\right)$ to be $\left(J_{0}, T_{0}, \zeta_{0}\right)$. Set $\tilde{S}=S, \tilde{j}: \tilde{S} \rightarrow M$ the immersion and $\tilde{R}$ a copy of $\tilde{R}_{+}$. The partial PW subsurface in $\tilde{M}$ can be picked as $(\tilde{S}, \tilde{R}, \tilde{j})$.

Case 2. If $J_{ \pm}$are both Seifert fibered, we need to recall the antennas property for graph manifolds, introduced in the proof of [PW1, Proposition 3.1].

For simplicity, let $N$ be a graph manifold with nonempty boundary that satisfies Proposition 2.1. Then in our notations, we say that $N$ has the antennas property, if for any two adjacent JSJ pieces $J_{0}, J_{1}$, there is a chunk $A$ of $N$, called an antenna, which is the union of consecutively adjacent distinct pieces $J_{0}, J_{1}, \cdots, J_{n}$ (more precisely, $J_{i} \cap J_{j}$ is a JSJ torus if $|j-i|=1$, and is empty otherwise), such that $J_{n}$ contains a boundary component of $N$.

In our discussion, we consider the maximal graph-manifold chunk $Q \subset M$ containing $T_{0}$ as a JSJ torus. It is implied by the proof of [PW1, Proposition 3.1] that there is a finite cover $\tilde{M}$ of $M$, such that any elevation $\tilde{Q}$ has the antennas property.

Note that $J_{ \pm} \subset Q$. Take elevations $\tilde{J}_{ \pm} \subset \tilde{Q}$ of $J_{ \pm}$adjacent along an elevation $\tilde{T}_{0}$ of $T_{0}$, and take an elevation $\tilde{\zeta}_{0} \subset \tilde{T}_{0}$ of $\zeta_{0}$. For simplicity, still denote $\tilde{J}_{ \pm}$and so on by $J_{ \pm}$and so on in this and further coverings. We take two antennas $A_{ \pm}$, starting with $J_{0}=J_{\mp}$ and $J_{1}=J_{ \pm}$, respectively. Passing to a finite cover of $\tilde{M}$ induced by a cover of its dual graph if necessary, we may assume $A_{+}$and $A_{-}$have no common JSJ piece other than $J_{ \pm}$, so we call $B=A_{+} \cup A_{-}$a bi-antennas throught $\tilde{T}_{0}$. We may further assume the dual graph of $\tilde{Q}$ has no cycle of at most three edges, then there is no JSJ piece of $\tilde{Q}$ adjacent to two JSJ pieces of $B$.

Denote $B_{ \pm}$the two parts of $B$ separated by $\tilde{T}_{0}$. We proceed to construct a properly embedded, incompressible, and boundary-incompressible subsurface $\tilde{E}^{*}$ such that $\tilde{E}^{*}$ intersects $\tilde{T}_{0}$ in slopes parallel to $\tilde{\zeta}_{0}$, and that $\tilde{T}_{0}$ cuts $\tilde{E}^{*}$ into two parts $\tilde{E}_{ \pm}^{*} \subset \tilde{Q}$. This follows the construction in [PW1, Proposition 3.1], as outlined below.

Start with $\tilde{\zeta}_{0}$ and try to extend a surface $\tilde{E}_{ \pm} \subset B_{ \pm}$. Due to the symmetry, we just discuss the extension on $B_{+}$. Suppose first $\tilde{\zeta}_{0}$ is not a fiber of $J_{1}$ up to isotopy. One can find a horizontal properly embedded incompressible subsurface $\tilde{E}_{+}$of $B_{+}$, such that $\tilde{E}_{+}$ intersects $\tilde{T}_{0}$ in parallel copies of $\tilde{\zeta}_{0}$. Moreover, if a component of $\partial \tilde{E}_{+}$does not lie on $\partial \tilde{Q}$, one can also make sure that it lies on a JSJ torus inside $\tilde{Q}$ parallel to the adjacent fiber. Take a pair of oppositely oriented parallel copies $\tilde{E}_{+}^{\uparrow}$ and $\tilde{E}_{+}^{\downarrow}$ of $\tilde{E}_{+}$. For each component $c \subset \partial \tilde{E}_{+}$that lies in a JSJ torus inside $\tilde{Q}$, one can find a properly embedded, boundary-essential vertical annulus in the adjacent piece bounding $c^{\uparrow} \cup c^{\downarrow}$, and glue this vertical annulus to $\tilde{E}_{+}^{\uparrow}$ and $\tilde{E}_{+}^{\downarrow}$ along the boundary, correspondingly. Since we assumed that no piece of $\tilde{Q}$ is adjacent to two JSJ pieces of $B$, the result is a properly embedded, 
incompressible, and boundary-incompressible subsurface $\tilde{E}_{+}^{*}$ as desired. Suppose then $\tilde{\zeta}_{0}$ is a fiber of $J_{1}$. Then two copies of $\tilde{\zeta}_{0}$ with opposite direction bound an essential vertical annulus in $J_{1}$ as we just discussed, which will be our $\tilde{E}_{+}=\tilde{E}_{+}^{*}$.

In general, $\tilde{E}^{*}$ is not closed, and $\partial \tilde{E}^{*}$ intersects $\partial \tilde{Q}$ in parallel slopes on each component that it reaches. Since $\tilde{Q}$ is a maximal graph-manifold chunk of $\tilde{M}$, for each such component as above, there is a geometrically finite, $\pi_{1}$-injectively immersed proper subsurface of the adjacent hyperbolic piece whose boundary finitely covers the corresponding slope. Performing the merging trick again, we obtain a PW subsurface $\tilde{j}: \tilde{S} \rightarrow \tilde{M}$, so that the part of $\tilde{S}$ inside $\tilde{Q}$ covers $\tilde{E}^{*}$. Note that the virtual embeddedness of $\tilde{S}$ in graphmanifold chunks follows from [PW2, Lemma 3.6]. In particular, $\tilde{S}$ intersects $\tilde{T}_{0}$ along covers of $\tilde{\zeta}_{0}$. Moreover, $\tilde{S}$ is cut by $\tilde{T}_{0}$ into two parts $\tilde{S}_{ \pm}$, and the part of $\tilde{S}_{ \pm}$inside $\tilde{Q}$ covers $\tilde{E}_{ \pm}^{*}$, respectively. We pick a connected component of $\tilde{S}_{+}$for $\tilde{R}$.

Now the triple $(\tilde{S}, \tilde{R}, \tilde{j})$ defines a partial PW subsurface of $\tilde{M}$ with respect to $\left(\tilde{J}_{0}, \tilde{T}_{0}, \tilde{\zeta}_{0}\right)$.

Case 3. Suppose one of $J_{ \pm}$is hyperbolic and the other is Seifert fibered. This case is a mixture of the previous two cases, and the construction is very similar, so we omit the details. In fact, this case was also covered by the construction of [PW2, Section 3], although not explicitly stated.

4.4. Virtual existence of corridor surfaces. The surfaces provided in the following theorem, later serving as corridor surfaces, will be crucial in proving Theorem 1.6 ,

Theorem 4.12. Let $M$ be an orientable closed irreducible mixed 3-manifold and let $\zeta_{0}$ be a slope on a JSJ torus $T_{0}$ adjacent to a JSJ piece $J_{0} \subset M$. Suppose $R \rightarrow M$ is a parallel-cutting partial $P W$ subsurface bounded virtually by $\zeta_{0}$ outside $J_{0}$. Then there exists a regular finite cover $\tilde{X}$ of the carrier chunk $X(R)$ in which every elevation of $R$ is embedded, intersecting any elevation of the carrier boundary $T_{0}$ in at most one slope.

The major part of the proof of Theorem 4.12 is the following weaker version Proposition 4.13 which is stated in a rather complicated form so that the application of Przytycki-Wise results, the merging process, and the intersection counting become more explicit in the proof. To state Proposition 4.13, we need some terminologies.

For any positive integer $m$, we construct a 2-complex $Y_{m}(R)$ immersed in $X(R)$ as follows. For each component $c \subset \partial R$, let $\tilde{T}_{0}^{m}(c)$ be a copy of a cover of $T_{0}$, in which there are exactly $m$ distinct elevations of $\zeta_{0}$, each of them covering $\zeta_{0}$ with the same degree as $c$. We glue $\tilde{T}_{0}^{m}(c)$ with $R$ naturally by identifying an elevation of $\zeta_{0}$ with $c \subset \partial R$. After the gluing for each boundary component of $R$, we get a 2-complex

$$
Y_{m}(R)=R \cup \bigcup_{c \subset \partial R} \tilde{T}_{0}^{m}(c),
$$

and there is a natural immersion

$$
Y_{m}(R) \rightarrow X(R),
$$

which is the immersion of the partial PW subsurface restricted to $R$, and is the covering of the boundary torus $T_{0}$ restricted to the tori $\tilde{T}_{0}^{m}(c)$. Note that $Y_{m}(R)$ is naturally defined in the sense that if we chose a different cover $\tilde{T}_{0}^{m}(c)$ or a different elevation of $\zeta_{0}$ for the gluing, the resulted immersion would differ only by a homeomorphism of $Y_{m}(R)$. If $R$ is oriented, so is $\partial R$ and its covers. 
Proposition 4.13. With the notations above and the oriented surface $R$, there exists an integer $m_{0}>0$ such that for any positive integral multiple $m$ of $m_{0}$, there is a regular finite cover $\tilde{X}$ of $X(R)$ in which every elevation $\tilde{Y}_{m}(R)$ of $Y_{m}(R)$ is embedded, and moreover $\tilde{\mathcal{R}} \cap \tilde{T}_{0}$ consists of $r$ directed parallel circles in $\tilde{T}_{0}$ induced from $\partial R$, where $\tilde{\mathcal{R}}$ is the union of elevations of $R$ contained in $\tilde{Y}_{m}(R), \tilde{T}_{0}$ is an elevation of $T_{0}$ contained in $\tilde{Y}_{m}(R)$, and $r$ is a positive integer.

To prove Proposition 4.13 , we need the following Lemma 4.14 .

There is a canonical (possibly disconnected) compact essential subsurface of $R$, called the horizontal part. It is the union of all subsurfaces of $R$ that are properly horizontally immersed in Seifert fibered pieces, glued up along the cut curves where any two are adjacent. Note that every complementary component of the union of the horizontal part and the cut curves is either a vertical cylinder immersed in a Seifert fibered piece, or a geometrically finite cusped subsurface immersed in a hyperbolic piece.

Lemma 4.14. For every component $F$ of the horizontal part of $R$, there is a unique graphmanifold chunk $Q_{F} \subset X(R)$ in which $F$ is properly horizontally immersed. Moreover, suppose $\tilde{Q}_{F}$ is a regular finite cover of $Q_{F}$ in which any elevation of $F$ is embedded, then for any elevation $\tilde{T}$ of a component $T \subset \partial Q_{F}$ and any elevation $\tilde{F}$ of $F$, the number of components of $\tilde{T} \cap \tilde{F}$ depends only on $T$ and $F$.

Proof. The first claim is direct: let $Q_{F}$ be the minimal subchunk of $X(R)$ containing the image of $F$, which is unique. Since $R$ is parallel cutting, it is clear that $\partial F$ cannot sit in any JSJ torus in the interior of $Q_{F}$, so $F$ is properly horizontally immersed in $Q_{F}$. Below we devote to the proof the "moreover" part.

Since $\tilde{Q}_{F}$ is a regular cover of $Q_{F}$, it suffices to fix an elevation $\tilde{F}$ and show that the number of components of $\tilde{T} \cap \tilde{F}$ is constant for all elevations $\tilde{T}$ of $T$. Let $f \subset T$ be a Seifert fiber of the adjacent JSJ piece of $Q_{F}$. As $R$ is parallel cutting, there is also a slope $s \subset T$ covered by all the components of $\partial F$ that are immersed in $T$. For any elevation $\tilde{T}$ of $T$, we may pick elevations $\tilde{f}, \tilde{s} \subset \tilde{T}$ of $f$ and $s$, respectively.

The (geometric) intersection numbers $i(\tilde{f}, \tilde{s})$ and $i(f, s)$ are related by the formula

$$
i(\tilde{f}, \tilde{s})=i(f, s) \cdot \frac{[\tilde{f}: f][\tilde{s}: s]}{[\tilde{T}: T]},
$$

where $[-:-]$ denotes the covering degree. This follows from $p^{-1}(f)=\frac{[\tilde{T}: T]}{[\tilde{f}: f]} \tilde{f}, p^{-1}(s)=$ $\frac{[\tilde{T}: T]}{[\tilde{s}: s]} \tilde{s}$ and $i\left(p^{-1}(f), p^{-1}(s)\right)=[\tilde{T}: T] i(f, s)$, where $p: \tilde{T} \rightarrow T$ is the discussed covering.

Since $\tilde{F}$ is horizontally embedded, $\tilde{Q}_{F}$ fibers over the circle with fiber $\tilde{F}$, each components of $\tilde{T} \cap \tilde{F}$ must be a copy of $\tilde{s}$. Hence the number of components of $\tilde{T} \cap \tilde{F}$ satisfies

$$
\left|\pi_{0}(\tilde{T} \cap \tilde{F})\right|=\frac{i(\tilde{f}, \tilde{F})}{i(\tilde{f}, \tilde{s})} .
$$

Thus it suffices to show $i(\tilde{f}, \tilde{F})$ is constant for all elevations $\tilde{f}$ of $f$.

By a calculation similar to formular (4.1) we have

$$
i(\tilde{f}, \tilde{F})=i(\tilde{f}, \partial \tilde{F})=i\left(\tilde{f}, p^{-1}(s)\right)=i(f, s)[\tilde{f}: f]
$$

Let $\Lambda$ and $\tilde{\Lambda}$ be the dual graph associated to the JSJ decompositions of $Q_{F}$ and $\tilde{Q}_{F}$. Note that there is a natural combinatorial map $\tilde{\Lambda} \rightarrow \Lambda$ induced by the covering. For any vertex $\tilde{v}$ of $\tilde{\Lambda}$, we write the corresponding JSJ piece of $\tilde{Q}_{F}$ as $\tilde{J}_{\tilde{v}}$, and the ordinary Seifert 
fiber of $\tilde{J}_{\tilde{v}}$ as $\tilde{f}_{\tilde{v}}$; for any edge $\tilde{e}$ of $\tilde{\Lambda}$, we write the corresponding JSJ torus as $\tilde{T}_{\tilde{e}}$, and the slope of $\tilde{T}_{\tilde{e}}$ parallel to the components of $\tilde{T}_{\tilde{e}} \cap \tilde{F}$ as $\tilde{s}_{\tilde{e}}$. The notations for $\Lambda$ are similar. As $R$ is parallel cutting, for any directed edge $e$ of $\Lambda$, the ratio

$$
\lambda_{e}=\frac{i\left(f_{\operatorname{ter}(e)}, s_{e}\right)}{i\left(f_{\operatorname{ini}(e)}, s_{e}\right)}
$$

is a positive rational number depending only on $F$ and $T_{e}$. Here ini $(e)$, ter $(e)$ denotes the initial vertex and the terminal vertex of $e$, respectively. Suppose $\tilde{e}_{1}, \cdots, \tilde{e}_{n}$ is a sequence of edges of $\tilde{\Lambda}$, consecutively joining the sequence of vertices $\tilde{v}_{0}, \cdots, \tilde{v}_{n}$ of $\tilde{\Lambda}$. We write $v_{k}, e_{k}$ for the image of $\tilde{v}_{k}, \tilde{e}_{k}$ under $\tilde{\Lambda} \rightarrow \Lambda$, respectively. From the formula (4.2), we have:

$$
\begin{aligned}
\frac{i\left(\tilde{f}_{\tilde{v}_{n}}, \tilde{F}\right)}{i\left(\tilde{f}_{\tilde{v}_{0}}, \tilde{F}\right)} & =\prod_{k=1}^{n} \frac{i\left(\tilde{f}_{\tilde{v}_{k}}, \tilde{F}\right)}{i\left(\tilde{f}_{\tilde{v}_{k-1}}, \tilde{F}\right)} \\
& =\prod_{k=1}^{n} \frac{i\left(f_{v_{k}}, s_{e_{k}}\right)}{i\left(f_{v_{k-1}}, s_{e_{k}}\right)} \cdot \frac{\left[\tilde{f}_{\tilde{v}_{k}}: f_{v_{k}}\right]}{\left[\tilde{f}_{\tilde{v}_{k-1}}: f_{v_{k-1}}\right]} \\
& =\frac{\left[\tilde{f}_{\tilde{v}_{n}}: f_{v_{n}}\right]}{\left[\tilde{f}_{\tilde{v}_{0}}: f_{v_{0}}\right]} \cdot \prod_{k=1}^{n} \lambda_{e_{k}} .
\end{aligned}
$$

In particular, if $\tilde{f}$ and $\tilde{f}^{\prime}$ are two elevations of the Seifert fiber $f$ on a given component $T \subset \partial Q_{F}$, we may pick a path as above so that $\tilde{f}_{\tilde{v}_{0}}=\tilde{f}$ and $\tilde{f}_{\tilde{v}_{n}}=\tilde{f}^{\prime}$. Since $v_{0}=v_{n}$ and $\tilde{Q}_{F}$ is a regular cover, $\left[\tilde{f}_{\tilde{v}_{n}}: f_{v_{n}}\right]=\left[\tilde{f}_{\tilde{v}_{0}}: f_{v_{0}}\right]$. Thus it suffices to show $\lambda_{e_{1}} \cdots \lambda_{e_{n}}=1$ for any cycle $e_{1}, \cdots, e_{n}$ of $\Lambda$.

To see this, note that $F$ is properly horizontally immersed in $Q_{F}$. If $e$ is a directed edge of $\Lambda$, then any component of $j^{-1}\left(J_{\operatorname{ini}(e)}\right) \subset F$ is adjacent to a component of $j^{-1}\left(J_{\operatorname{ter}(e)}\right) \subset F$. Therefore, if $e_{1}, \cdots, e_{n}$ is a cycle of $\Lambda$, then starting with any component $C \subset j^{-1}\left(J_{v_{0}}\right)$, we may find a path $\gamma:[0,1] \rightarrow F$ so that $\gamma(0)$ lies in $C$, consecutively intersects $T_{e_{1}}, \cdots, T_{e_{n}}$, and $\gamma(1)$ lies in a component $C^{\prime} \subset j^{-1}\left(J_{v_{0}}\right)$. Since there are only finitely many components of $j^{-1}\left(J_{v_{0}}\right)$, we may join a number of such $\gamma$ 's as above to obtain a loop $S^{1} \rightarrow F$ which goes around the cycle for a positive number of times, say $r$ times. Because $F \rightarrow Q_{F}$ is a proper horizontal immersion which is a virtual embedding, it follows from the criterion of Rubinstein-Wang [RW] Theorem 2.3] that

$$
\left(\lambda_{e_{1}} \cdots \lambda_{e_{n}}\right)^{r}=1
$$

Therefore, $\lambda_{e_{1}} \cdots \lambda_{e_{n}}=1$ and this completes the proof.

Proof of Proposition 4.13 Since one cannot directly claim that $Y_{m}(R)$ is $\pi_{1}$-injectively immersed in $X(R)$ (indeed this is not necessarily true in general), we will prove the proposition by the following strategy: First for virtually all positive integer $m$, by using the results of Przytycki and Wise, as well as Lemma 4.14, we can virtually embed $Y_{m}(R)$ into a compact 3-manifold $\mathcal{Y}_{m}^{*}$ and make sure that $\mathcal{Y}_{m}^{*} \rightarrow X(R)$ is virtually embedded when restricted on each JSJ piece. And finally we will apply the merging trick (Proposition 4.2) to get the global embedding in the Proposition 4.13. For simplicity, we write $X$ for $X(R)$ and $Y_{m}$ for $Y_{m}(R)$.

For the triple $(S, R, j)$ associated to the partial PW subsurface $R \rightarrow M$, there is a regular finite cover of $M$ in which any elevation of $S$ is embedded, by the separability of PW subsurfaces (Lemma 4.7). Hence we may assume $X^{\prime}$ is a regular finite cover of $X$ in 
which any elevation of $R$ is embedded. Let

$$
R^{\prime} \subset X^{\prime}
$$

be an elevation of $R$. Take a compact regular neighborhood of the horizontal part of $R^{\prime}$, and for each cut curve or boundary curve not adjacent to the horizontal part, take a compact regular neighborhood of it, and make sure these regular neighborhoods are mutually disjoint. Let $\mathcal{F}^{\prime} \subset R^{\prime}$ be the union of these regular neighborhoods. For each component $F^{\prime} \subset \mathcal{F}^{\prime}$, let $\mathcal{Q}_{F^{\prime}}^{\prime} \subset X^{\prime}$ be a compact regular neighborhood of a chunk, or of a JSJ or boundary torus, so that $F^{\prime}$ is properly embedded in $\mathcal{Q}_{F^{\prime}}^{\prime}$. Hence $\mathcal{Q}_{F^{\prime}}^{\prime}$ is a bundle over the circle with fibre $F^{\prime}$, denoted as $\left(F^{\prime}, \phi_{F^{\prime}}\right)$, where $\phi_{F^{\prime}}: F^{\prime} \rightarrow F^{\prime}$ is the gluing map, which is a periodic on each reducible piece in the sense of Nielsen-Thurston.

By Lemma 4.14 and some straighforward verification, for a JSJ or boundary torus $T \subset$ $X$ and a component $F^{\prime} \subset \mathcal{F}^{\prime}$, any elevation $T^{\prime}$ of $T$ intersects $F^{\prime}$ if and only if $T^{\prime} \subset \mathcal{Q}_{F^{\prime}}^{\prime}$; and furthermore, such $T^{\prime}$ intersects $F^{\prime}$ in a number $\mu_{T}\left(F^{\prime}\right)>0$ of components, depending only of $T$ and $F^{\prime}$ (indeed, only on the subsurface of $R$ that $F^{\prime}$ covers). As $R$ is parallel cutting, let $s \subset T$ be the slope covered by the components of $F^{\prime}$. Note that $s$ is $\zeta_{0}$ if $T$ is $T_{0}$. Let

$$
k_{T}^{\prime}=\frac{\left[T^{\prime}: T\right]}{\left[s^{\prime}: s\right]}
$$

be the number of elevations of $s$ in any elevation of $T$, which is well defined since $X^{\prime}$ is a regular finite cover of $X$. Let

$$
m_{0}^{\prime}>0
$$

be the product of the least common multiple of all $k_{T}^{\prime}$ and the least common multiple of all $\mu_{T}\left(F^{\prime}\right)$. For any positive multiple $m$ of $m_{0}^{\prime}$, let $p_{*}: \mathcal{Q}_{F^{\prime}}^{*} \rightarrow \mathcal{Q}_{F^{\prime}}^{\prime}$ be the cyclic covering dual to $F^{\prime}$, of degree

$$
d=\left[\mathcal{Q}_{F^{\prime}}^{*}: \mathcal{Q}_{F^{\prime}}^{\prime}\right]=m \cdot \frac{\mu_{T_{0}}\left(F^{\prime}\right)}{k_{T_{0}}^{\prime}},
$$

or just $m$ if $\mathcal{Q}_{F^{\prime}}^{*}$ contains no elevations of $T_{0}$.

Clearly $\mathcal{Q}_{F^{\prime}}^{*}=\left(F^{\prime}, \phi_{F^{\prime}}^{d}\right)$. Since $d$ is a multiple of each $\mu_{T_{0}}\left(F^{\prime}\right)$, each component of $\partial F^{\prime}$ (also cutting curves) is invariant under $\phi_{F^{\prime}}^{d}$. Therefore each component of $\partial \mathcal{Q}_{F^{\prime}}^{*}$ contains one and only one component of $\partial F^{\prime}$. Note that $p_{*}^{-1}\left(F^{\prime}\right)$ has $d$ components in $\mathcal{Q}_{F^{\prime}}^{*}$ and each one is a lift of $F^{\prime}$; moreover for each $T_{0}^{\prime}$ covering $T_{0}, p_{*}^{-1}\left(T_{0}^{\prime}\right)$ has $\mu_{T_{0}}\left(F^{\prime}\right)$ components. Let $p_{0}: T_{0}^{\prime} \rightarrow T_{0}$ be the discussed covering, then $p_{0}{ }^{-1}\left(\zeta_{0}\right)$ has $k_{T_{0}}^{\prime}$ components in $T_{0}^{\prime}$, therefore $\left(p_{*} \circ p_{0}\right)^{-1}\left(\zeta_{0}\right)$ has $d K_{T_{0}}^{\prime}$ components on $\partial \mathcal{Q}_{F^{\prime}}^{*}$. It follows that in every component of $\partial \mathcal{Q}_{F^{\prime}}^{*}$ that covers $T_{0}$, there are exactly $\frac{d K_{T_{0}}^{\prime}}{\mu_{T_{0}}\left(F^{\prime}\right)}=m$ elevations of $\zeta_{0}$,

Let $\mathcal{W}_{R^{\prime}}^{\prime} \subset X^{\prime}$ be a compact regular neighborhood of $R^{\prime}$, and let $\mathcal{W}_{F^{\prime}}^{*} \subset \mathcal{Q}_{F^{\prime}}^{*}$ be a compact regular neighbood of a fiber $F^{\prime}$. Since $\mathcal{W}_{R^{\prime}}^{\prime} \cap \mathcal{Q}_{F^{\prime}}^{\prime}$ is naturally homeomorphic to $\mathcal{W}_{F^{\prime}}^{*}$, we may take a copy of $\mathcal{W}_{R^{\prime}}^{\prime}$ and glue it with a copy of $\mathcal{Q}_{F^{\prime}}^{*}$ by identifying $\mathcal{W}_{R^{\prime}}^{\prime} \cap \mathcal{Q}_{F^{\prime}}^{\prime}$ and $\mathcal{W}_{F^{\prime}}^{*}$, for all components $F^{\prime} \subset \mathcal{F}^{\prime}$. The result is a compact 3-manifold

$$
\mathcal{Y}_{m}^{*}=\mathcal{W}_{R^{\prime}}^{\prime} \cup \bigcup_{F^{\prime} \subset \mathcal{F}^{\prime}} \mathcal{Q}_{F^{\prime}}^{*}
$$

with boundary, and there is a natural immersion

$$
\varphi: \mathcal{Y}_{m}^{*} \leftrightarrow X^{\prime}
$$

which sends $W_{R^{\prime}}^{\prime}$ to $W_{R^{\prime}}^{\prime} \subset X^{\prime}$ by the identity and each $\mathcal{Q}_{F^{\prime}}^{*}$ to $\mathcal{Q}_{F^{\prime}}^{\prime} \subset X^{\prime}$ via the given covering. Moreover, the union of the copy $R^{\prime} \subset \mathcal{Y}_{m}^{*}$ and all components of $\partial \mathcal{Q}_{F^{\prime}}^{\prime}$ that 
cover $T_{0}$ is an embedded 2-complex

$$
Y_{m}^{*} \subset \mathcal{Y}_{m}^{*}
$$

Moreover, $Y_{m}^{*}$ naturally covers $Y_{m}$ with degree $\left[R^{\prime}: R\right]$, and the hanging tori $\tilde{T}_{0}^{m}(c)$ all lift. In other words, $Y_{m}$ is virtually embedded in $\mathcal{Y}_{m}^{*}$, naturally in the sense that the map $Y_{m}^{*} \rightarrow$ $X^{\prime}$ induced from the immersions of $Y_{m}$ and of $\mathcal{Y}_{m}^{*}$ are the same up to homeomorphism of $Y_{m}^{*}$.

We are going to show that $\varphi: \mathcal{Y}_{m}^{*} \rightarrow X^{\prime}$ is a virtual embedding. To do this we first show that the restriction on each JSJ piece of $\mathcal{Y}_{m}^{*}$ is a virtual embedding.

The JSJ tori of $\mathcal{Y}_{m}^{*}$ are exactly the JSJ or boundary tori of all $\mathcal{Q}_{F^{\prime}}^{*}$. The JSJ pieces of $\mathcal{Y}_{m}^{*}$ are the JSJ pieces of $\mathcal{Q}_{F^{\prime}}^{*}$, and the pieces containing the components of $R^{\prime} \backslash \mathcal{F}^{\prime}$. To describe the latter type more precisely, consider any connected compact subsurface $V^{\prime} \subset R^{\prime}$, which is properly immersed in a JSJ piece of $X^{\prime}$ and vertically or geometrically finite. For each such $V^{\prime}$, there is a unique component of $R^{\prime} \backslash \mathcal{F}^{\prime}$ contained in $V^{\prime}$, which is isotopic to the interior of $V^{\prime}$. Then the unique JSJ piece of $\mathcal{Y}_{m}^{*}$ containing $V^{\prime}$ is the piece bounded by all the JSJ tori adjacent to $\partial V^{\prime}$, and this piece deformation retracts to the union of $V^{\prime}$ and all its adjacent JSJ tori, denoted by $Y^{*}\left(V^{\prime}\right)$. In fact, $Y^{*}\left(V^{\prime}\right)$ can be described in a similar fashion as that of $Y_{m}(R)$. For each component $c^{\prime} \subset \partial V^{\prime}$ that covers a slope in a torus $T \subset X$, we glue a copy of a cover $\tilde{T}^{m\left(c^{\prime}\right)}\left(c^{\prime}\right)$ of $T$ to $V$ along $c$ in a similar way as $\tilde{T}_{0}^{m}(c)$. Explicitly, since there is a unique $F^{\prime} \subset \mathcal{F}^{\prime}$ containing $c^{\prime}$,

$$
m\left(c^{\prime}\right)=\left[\mathcal{Q}_{F^{\prime}}^{*}: \mathcal{Q}_{F^{\prime}}^{\prime}\right] \cdot \frac{k_{T}^{\prime}}{\mu_{T}\left(F^{\prime}\right)},
$$

which is a positive integer by our choice of $m_{0}^{\prime}$.

Suppose $V^{\prime} \subset R^{\prime}$ is a vertical or geometrically finite subsurface as above, and $K^{*} \subset$ $\mathcal{Y}_{m}^{*}$ is the unique JSJ piece containing $V^{\prime}$. As we have seen, the inclusion of the 2-complex $Y^{*}\left(V^{\prime}\right) \hookrightarrow K^{*}$ is a homotopy equivalence. Let $J^{\prime} \subset X^{\prime}$ be the JSJ piece in which $K^{*}$ is immersed into, so that there is an induced immersion $\varphi \mid: Y^{*}\left(V^{\prime}\right) \rightarrow J^{\prime}$. If $J^{\prime}$ is hyperbolic, then by [PW3, Theorem 4.1], $\varphi$ restricted to $Y^{*}\left(V^{\prime}\right)$ is $\pi_{1}$-injective and relatively quasiconvex if all $m\left(c^{\prime}\right)$ above are sufficiently large; and in this case, it is a consequence of the relative quasiconvex separability due to Wise [Wi, Theorem 16.23] (cf. [PW3, Corollary 4.2]) that $\pi_{1}\left(Y^{*}\left(V^{\prime}\right)\right)$ is indeed separable. Then we may find a finite cover of $J^{\prime}$ in which the elevations of $Y^{*}\left(V^{\prime}\right)$, and hence elevations of $K^{*}$, are embedded.

If $J^{\prime}$ is Seifert fibered, then $J^{\prime}$ is a product and $Y^{*}\left(V^{\prime}\right)$ is just the union of a properly immersed (boundary-essential) vertical annulus together with covers of the tori that are adjacent to. If all $m\left(c^{\prime}\right)$ are sufficiently large, one can easily see that $\pi_{1}\left(Y^{*}\left(V^{\prime}\right)\right)$ is embedded in $\pi_{1}\left(J^{\prime}\right)$ and is separable. Then we may again find a finite cover of $J^{\prime}$ in which elevations of $Y^{*}\left(V^{\prime}\right)$, and hence elevations of $K^{*}$, are embedded. From the formula of $m\left(c^{\prime}\right)$ above, it is clear that $m\left(c^{\prime}\right)$ can be arbitrarily as large as desired if $m$ is sufficiently large. Therefore, we may pick

$$
m_{0}>0
$$

to be a sufficiently large multiple of $m_{0}^{\prime}$, so that any multiple of $m$ is sufficiently large to ensure that the $\pi_{1}$-injectivity and separability of $Y^{*}\left(V^{\prime}\right)$ work.

Note that if $K^{*}$ is a JSJ piece of $\mathcal{Y}_{m}^{*}$ that is contained in some $\mathcal{Q}^{*}\left(F^{\prime}\right)$, it covers a JSJ piece $J^{\prime}$ of $X^{\prime}$.

Therefore, we have shown that for every JSJ piece $K^{*} \subset \mathcal{Y}_{m}^{*}$, there is a JSJ piece $J^{\prime}$ of $X^{\prime}$ that contains the immersed image of $K^{*}$, and moreover we have an embedding $\phi^{\prime \prime}: K^{\prime \prime} \rightarrow J_{K^{*}}$ which covers $\varphi \mid: K^{*} \rightarrow J^{\prime}$. Now for each $J_{i}^{\prime} \subset X^{\prime}$, if $J_{i}^{\prime}$ contains the image of a JSJ piece $K^{*} \subset \mathcal{Y}_{m}^{*}$, let $J_{i}^{*}$ be the common finite cover of all those $J_{K^{*}}$, and 
otherwise set $J_{i}^{*}=J_{i}^{\prime}$. By Proposition 4.2, we have a regular finite JSJ $l$-characteristic covering $p^{\prime \prime}: X^{\prime \prime} \rightarrow X^{\prime}$ such that each covering $J^{\prime \prime} \rightarrow J_{i}^{\prime}$ factor through $J_{i}^{*}$.

Let $\varphi^{\prime \prime}: \mathcal{Y}_{m}^{\prime \prime} \rightarrow X^{\prime \prime}$ be any elevation of $\varphi: \mathcal{Y}_{m}^{*} \rightarrow X^{\prime}$. Since the virtual embeddedness is preserved under passage to further covers, $\varphi^{\prime \prime} \mid$ on each JSJ piece is an embedding. It follows that the induced map on the dual graph $\Lambda\left(\mathcal{Y}_{m}^{\prime \prime}\right) \rightarrow \Lambda\left(X^{\prime \prime}\right)$ is a combinatorial local embedding, which is $\pi_{1}$-injective. Because $\pi_{1}\left(\Lambda\left(X^{\prime \prime}\right)\right)$ is a free group, and hence is LERF, it has a regular finite cover in which any elevation of $\Lambda\left(\mathcal{Y}_{m}^{\prime \prime}\right)$ is an embedded subgraph. Therefore, we have a regular finite JSJ 1 -characteristic covering $p^{\prime \prime \prime}: X^{\prime \prime \prime} \rightarrow X^{\prime \prime}$ so that any elevation $\varphi^{\prime \prime \prime}: \mathcal{Y}_{m}^{\prime \prime \prime} \rightarrow X^{\prime \prime \prime}$ of $\varphi: \mathcal{Y}_{m}^{*} \rightarrow X^{\prime}$ is an embedding. As we discussed in Proposition 4.2 and its remark, by passing to a further finite cover $\tilde{X}$, we can assume that the JSJ $l$-characteristic covering $\tilde{X} \rightarrow X^{\prime}$ is characteristic in the usual sense, which implies that the covering $\tilde{X} \rightarrow X^{\prime} \rightarrow X$ is a finite regular covering.

In conclusion, for any positive multiple $m$ of the $m_{0}$ we have chosen, there is a regular finite cover $\tilde{X}$ of $X$, in which any elevation of $\mathcal{Y}_{m}^{*}$, and hence any elevation $\tilde{Y}$ of $Y_{m}$, is embedded.

Now we are going to prove the "moreover part": We fix an orientation of $R$. Let $m$ be a positive integer ensured by the first half of Proposition 4.13, so that $Y_{m}(R) \rightarrow X(R)$ is a virtual embedding. We assume $\tilde{X}$ is a regular finite cover of $X(R)$ constructed in Proposition 4.13 in which any elevation $\tilde{Y}$ of $Y_{m}(R)$ is embedded.

Note that there is a copy of $R$ contained in $Y_{m}(R)$, so for any elevation $\tilde{Y} \subset \tilde{X}$, there is a collection of mutually disjoint, embedded elevations of $R$, with naturally induced orientations. We fix an elevation $\tilde{Y}$ of $Y$, and let $\tilde{\mathcal{R}} \subset \tilde{Y}$ be the union of elevations of $R$ contained in $\tilde{Y}$.

Since $\zeta_{0}$ has $\frac{\left[\tilde{T}_{0}: T_{0}\right]}{\left[\tilde{\zeta}_{0}: \zeta_{0}\right]}$ elevations in any elevation $\tilde{T}_{0}$ of $T_{0}$, and $\zeta_{0}$ has $m$ elevations in $T_{0}^{m}(c) \subset Y_{m}(R)$ for each component $c$ of $\partial R$, it follows that $c \subset T_{0}^{m}(c) \subset Y_{m}(R)$ has $r=\frac{\left[\tilde{T}_{0}: T_{0}\right]}{m\left[\tilde{\zeta}_{0}: \zeta_{0}\right]}$ elevations in $\tilde{T}_{0}$. Since $R \subset Y_{m}(R)$ meets $T_{0}^{m}(c)$ exactly on $c$, it follows that for any elevation $\tilde{T}_{0}$ of $T_{0}$ contained in $\tilde{Y}$, there are exactly $r$ components of $\partial \tilde{\mathcal{R}} \cap \tilde{T}_{0}$. Furthermore, it is clear from the construction of $Y_{m}(R)$ that for any $\tilde{T}_{0} \subset \tilde{Y}$, all components of $\partial \tilde{\mathcal{R}} \cap \tilde{T}_{0}$ cover the same component of $\partial R$, and in particular, they are directly parallel on $\tilde{T}_{0}$ with the direction induced from $\partial \mathcal{R}$. Note also that for any $\tilde{T}_{0}$ not contained in $\tilde{Y}, \tilde{T}_{0} \cap \tilde{\mathcal{R}}$ is the empty set.

This completes the proof of Proposition 4.13 .

Proof of Theorem 4.12 We start from the conclusion of Proposition 4.13 To match the notations, still denote by $X^{\prime}, \mathcal{R}^{\prime}, Y^{\prime}$ and $T_{0}^{\prime}$ the spaces $\tilde{X}, \tilde{\mathcal{R}}, \tilde{Y}$ and $\tilde{T}_{0}$ obtained in Proposition 4.13, where everything is oriented.

The oriented properly embedded subsurface $\mathcal{R}^{\prime}$ represents a class $\left[\mathcal{R}^{\prime}\right] \in$ $H_{2}\left(X^{\prime}, \partial X^{\prime} ; \mathbb{Z}\right)$. Then the homological pairing with $\left[\mathcal{R}^{\prime}\right]$ induces a quotient homomorphism

$$
l_{\mathcal{R}^{\prime}}: H_{1}\left(X^{\prime} ; \mathbb{Z}\right) \stackrel{\left[\mathcal{R}^{\prime}\right]}{\longrightarrow} \mathbb{Z} \rightarrow \mathbb{Z}_{r} .
$$

Denote the group of deck transformations of the covering of $X^{\prime} \rightarrow X$ as $\Gamma_{X^{\prime}}$ and by taking the direct sum of all $\tau^{*}\left(l_{\mathcal{R}^{\prime}}\right)$, where $\tau$ runs over $\Gamma_{X^{\prime}}$ we define a homomorphism of integral modules:

$$
L=\oplus_{\tau \in \Gamma_{X^{\prime}}} \tau^{*}\left(l_{\mathcal{R}^{\prime}}\right)=\oplus_{\tau \in \Gamma_{X^{\prime}}} l_{\tau\left(\mathcal{R}^{\prime}\right)}: H_{1}\left(X^{\prime} ; \mathbb{Z}\right) \rightarrow \mathbb{Z}_{r}^{\oplus \operatorname{Gal}\left(X^{\prime}\right)}
$$


The kernel of the homomorphism of groups

$$
\kappa^{\prime}: \pi_{1}\left(X^{\prime}\right) \longrightarrow H_{1}\left(X^{\prime} ; \mathbb{Z}\right) \stackrel{L}{\longrightarrow} \mathbb{Z}_{r}^{\oplus \operatorname{Gal}\left(X^{\prime}\right)}
$$

is invariant under the deck transformation group $\Gamma_{X^{\prime}}$, thus it follows that $\operatorname{Ker}\left(\kappa^{\prime}\right) \subset$ $\pi_{1}\left(X^{\prime}\right) \subset \pi_{1}(X)$ is a normal subgroup, so it defines a regular finite cover

$$
\kappa: \tilde{X} \rightarrow X,
$$

which factors through $X^{\prime}$.

It remains to verify that every elevation $\tilde{R} \subset \tilde{X}$ of $R$ intersects any elevation $\tilde{T}_{0} \subset \tilde{X}$ of $T_{0}$ in at most one components. Since $\tilde{X}$ is a regular cover, we may assume $\tilde{R}$ is an elevation of a component $R^{\prime} \subset \mathcal{R}^{\prime}$. Thus, an elevation $\tilde{T}_{0}$ of $T_{0}$ intersects $\tilde{R}$ if and only if it covers an elevation $T_{0}^{\prime} \subset X^{\prime}$ of $T_{0}$ contained in $Y^{\prime}$. If there were at least two components of $\tilde{T}_{0} \cap \tilde{R}$ then we could pick two points $\tilde{x}, \tilde{y}$ on two distinct components, and there would be a directed loop $\tilde{\alpha}$ formed by two consecutive directed paths $\tilde{\alpha}_{\tilde{T}_{0}} \subset \tilde{T}_{0}$ and $\tilde{\alpha}_{\tilde{R}} \subset \tilde{R}$, both joining $\tilde{x}$ and $\tilde{y}$. Because $\tilde{R}$ and $\tilde{T}_{0}$ cover $R^{\prime}$ and $T_{0}^{\prime}$, respectively, and $\tilde{R} \subset \tilde{X}$ is a two-sided proper embedded surface, we may perturb $\tilde{\alpha}$ a bit so that $\tilde{\alpha}_{\tilde{R}}$ is projected into $X^{\prime}$ missing the interior of $\mathcal{R}^{\prime}$. Because the algebraic intersection number of $\tilde{R}$ and $\tilde{\alpha}$ is always an integer multiple of $r$, it follows that the path $\tilde{\alpha}_{\tilde{T}_{0}}$ is immersed under the covering into $T_{0}^{\prime}$, and has the algebraic intersection number with the components of $R^{\prime} \cap T_{0}^{\prime}$ an integral multiple of $r$. Because there are exactly $r$ components of $R^{\prime} \cap T_{0}^{\prime}$, directly parallel on $T_{0}^{\prime}$, this means that $\tilde{x}$ and $\tilde{y}$ are projected to the same component of $T_{0}^{\prime} \cap R^{\prime}$. Up to homotopy, we may assume they are the same, so $\tilde{\alpha}_{\tilde{T}_{0}}$ is the lift of a closed path $\alpha_{T_{0}^{\prime}}^{\prime} \rightarrow T_{0}^{\prime}$. However, since $\mathcal{R}^{\prime}$ intersects any elevation $T_{0}^{\prime}$ in either the empty set or exactly $r$ directly parallel components, $L$ vanishes on $H_{1}\left(T_{0}^{\prime} ; \mathbb{Z}\right)$ for any $T_{0}^{\prime}$. In other words, every $T_{0}^{\prime}$ lifts into $\tilde{X}$. This means that the closed path $\alpha_{T_{0}^{\prime}}^{\prime}$ lifts into $\tilde{T}_{0}$ as well, so $\tilde{x}$ and $\tilde{y}$ are the same. This contradicts the assumption that they lie on distinct components of $\tilde{R} \cap \tilde{T}_{0}$. We conclude that $\tilde{X}$ is the regular finite cover as desired.

\section{VIRTUAL EXTENSION OF REPRESENTATIONS}

In this section, we construct virtual extension of a representation $\rho_{0}: \pi_{1}\left(J_{0}\right) \rightarrow \mathscr{G}$ of a JSJ piece $J$ of a mixed 3-manifold $M$ assuming that the representation $\rho_{0}$ has nontrivial kernel on $\pi_{1}(T)$ for each torus $T \subset \partial J_{0}$ (Theorem 5.2). For the sake of generality, we abstract a property of the target group $\mathscr{G}$ called class invertibility (Definition 5.1), with

which we can "flip" $\rho_{0}$ up to conjugation. In particular, $\operatorname{PSL}(2 ; \mathbb{C})$ and $\operatorname{Iso}_{e} \widehat{\mathrm{SL}_{2}(\mathbb{R})}$ are both class invertible (Lemma 6.1).

Definition 5.1. Let $\mathscr{G}$ be a group, and $\left\{\left[A_{i}\right]\right\}_{i \in I}$ be a collection of conjugacy classes of abelian subgroups. By a class inversion with respect to $\left\{\left[A_{i}\right]\right\}_{i \in I}$, we mean an outer automorphism $[\nu] \in \operatorname{Out}(\mathscr{G})$, such that for any representative abelian subgroup $A_{i}$ of each $\left[A_{i}\right]$, there is a representative automorphism $\nu_{A_{i}}: \mathscr{G} \rightarrow \mathscr{G}$ of $[\nu]$ that preserves $A_{i}$, taking every $a \in A_{i}$ to its inverse. We say $\mathscr{G}$ is class invertible with respect to $\left\{\left[A_{i}\right]\right\}_{i \in I}$, if there exists class inversion. We often ambiguously call any collection of representative abelian subgroups $\left\{A_{i}\right\}_{i \in I}$ a class invertible collection, and call any representative automorphism $\nu$ a class inversion.

Theorem 5.2. Let $\mathscr{G}$ be a group, and $M$ be an irreducible orientable closed mixed 3manifold. For a geometric piece $J_{0} \subset M$, suppose a representation

$$
\rho_{0}: \pi_{1}\left(J_{0}\right) \rightarrow \mathscr{G}
$$


satisfies the following:

- for every boundary torus $T \subset \partial J_{0}, \rho_{0}$ has nontrivial kernel restricted to $\pi_{1}(T)$; and

- for all boundary tori $T \subset \partial J_{0}, \rho_{0}\left(\pi_{1}(T)\right)$ form a class invertible collection of abelian subgroups of $\mathscr{G}$.

Then there exist a finite regular cover

$$
\kappa: \tilde{M} \rightarrow M
$$

and a representation

$$
\tilde{\rho}: \pi_{1}(\tilde{M}) \rightarrow \mathscr{G}
$$

satisfying the following:

- for one or more elevations $\tilde{J}_{0}$ of $J_{0}$, the restriction of $\tilde{\rho}$ to $\pi_{1}\left(\tilde{J}_{0}\right)$ is, up to a class inversion, conjugate to the pull-back $\kappa^{*}\left(\rho_{0}\right)$; and

- for any elevation $\tilde{J}$ other than the above, of any geometric piece $J$, the restriction of $\tilde{\rho}$ to $\pi_{1}(\tilde{J})$ is cyclic, possibly trivial.

The rest of this section is devoted to the proof of Theorem 5.2. In Subsection 5.1 we construct a cover of $M$ by merging colored chunks. In Subsection 5.2, each colored chunk will be endowed naturally with a representation, up to conjugation. Then the colored merging gives rise to a virtual representation as desired. There will be three colors 0 (null), +1 (positive), and -1 (negative), to be assigned to the boundary components of the chunks in our construction. To keep in mind, the null color will mean that the restricted representation to the boundary is trivial, and the signed colors will mean that the restricted representation to the boundary is nontrivial and the sign indicates whether a class inversion will be applied.

5.1. Colored chunks and colored merging. Let $M$ be an orientable closed irreducible mixed 3-manifold containing no essential Klein bottles. Let

$$
J_{0} \subset M
$$

be a selected JSJ piece of $M$. The boundary of $J_{0}$ is a disjoint union of tori:

$$
\partial J_{0}=\bigsqcup_{i=1}^{s} T_{i}
$$

and for each $T_{i}$, let

$$
\zeta_{i} \subset T_{i}
$$

be a selected slope. Fix a direction for each $\zeta_{i}$. With this data, we construct a regular finite cover $\tilde{M}$ of $M$ by merging colored chunks as follows.

By Theorem 4.11, for each $T_{i} \subset M$, there is a finite cover $M_{i}^{\prime}$ of $M$, and an elevation $\left(J_{0, i}^{\prime}, T_{i}^{\prime}, \zeta_{i}^{\prime}\right)$ of the triple $\left(J_{0}, T_{i}, \zeta_{i}\right)$, and there is a parallel-cutting partial PW subsurface $R_{i}^{\prime} \stackrel{\rightarrow}{\rightarrow} M_{i}^{\prime}$ virtually bounding $\zeta_{i}^{\prime}$ outside $J_{0, i}^{\prime}$. By Theorem 4.12, there is a regular finite cover $X_{i}^{\prime \prime}$ of the carrier chunk $X\left(R_{i}^{\prime}\right) \subset M_{i}^{\prime}$, in which any elevation of $R_{i}^{\prime}$ is properly embedded, intersecting each elevation of the carrier boundary $T_{i}^{\prime}$ in at most one slope. Pick an elevation $R_{i}^{*} \subset X_{i}^{\prime \prime}$ of $R_{i}^{\prime}$, and let $X_{i}^{*} \subset X_{i}^{\prime \prime}$ be the carrier chunk of $R_{i}^{*}$, namely, the minimal chunk containing $R_{i}^{*}$. Taking a copy of $X_{i}^{*}$ together with $R_{i}^{*}$, we call the (abstract) duple $\left(X_{i}^{*}, R_{i}^{*}\right)$ a corridor chunk associated to the sloped boundary $\left(T_{i}, \zeta_{i}\right)$. The properly embedded subsurface $R_{i}^{*}$ is called the corridor surface of $X_{i}^{*}$, and the corridor boundary $\partial^{*} X_{i}^{*}$ of $X_{i}^{*}$ is the union of boundary components that intersect $R_{i}^{*}$. Note that every corridor boundary component is an elevation of $T_{i}$. To color the boundary of $X_{i}^{*}$, 
we pick an orientation of $R_{i}^{\prime}$. For any corridor boundary component $T^{*} \subset \partial^{*} X_{i}^{*}, R_{i}^{*}$ intersects $T^{*}$ in exactly one slope $c^{*}$, and $c^{*}$ has one direction induced from the direction of $\zeta_{i}$, and another direction induced from the orientation of $R_{i}^{*}$. We color any corridor boundary component $T^{*}$ by +1 if these two induced directions of $c^{*}$ agree, or by -1 otherwise. We color any non-corridor boundary component of $X_{i}^{*}$ by 0 . The result is a colored corridor chunk

$$
\left(X_{i}^{*}, R_{i}^{*}\right) \text {. }
$$

Note that $X_{i}^{*}$ has the same number of positively colored boundary components and negatively colored components.

A copy of $J_{0}$ with boundary components colored all by +1 , or all by -1 , is called a positively colored $J_{0}$ piece, or a negatively colored $J_{0}$ piece, respectively.

A copy of any JSJ piece $J \subset M$ (possibly $J_{0}$ ) with all boundary components colored by 0 is called a null colored JSJ piece.

By an elevated colored chunk, we mean a finite cover of any of the following:

- a positively or negatively colored $J_{0}$ piece

- a colored corridor chunk associated to a $\left(T_{i}, \zeta_{i}\right)$

- a null colored JSJ piece from $M$

together with the naturally induced boundary coloring.

Lemma 5.3. With the notations above, there exists a regular finite cover $\tilde{M}$ of $M$, obtained by gluing elevated colored chunks along boundary tori matching the coloring. Moreover, $\tilde{M}$ contains at least one elevated positively colored $J_{0}$ piece.

Proof. Because every colored chunk is naturally a semicover of $M$ (Definition 4.4), applying Corollary 4.5 and Proposition 4.2, there exists a positive integer $m$, so that for each colored chunk there is an elevated colored chunk semicovering $M$ and inducing the $m$ characteristic covering on the boundary. Suppose $\widehat{J}$ is such a cover of $J$ for any JSJ piece $J \subset M$, and $\widehat{X}_{i}^{*}$ is such a cover for any $X_{i}^{*}$.

Suppose $\widehat{X}_{i}^{*}$ has $k_{i}$ positively colored boundary components, and hence $k_{i}$ negatively colored boundary components. Suppose $\widehat{J}_{0}$ has $l_{i}$ boundary components that are elevations of $T_{i}$. Let $K$ be the least common multiple of all $k_{i}$. We take $K$ copies of positively colored $\widehat{J}_{0}, K$ copies of negatively colored $\widehat{J}_{0}$, and $\frac{l_{i} K}{k_{i}}$ copies of each $\widehat{X}_{i}^{*}$. Then for each $i=1, \ldots, s$, the number of positively colored elevations of $T_{i}$ match from both sides and the same holds for negatively colored elevations of $T_{i}$. Thus we may glue these copies along their boundary, matching the coloring, and pick one component of the result to obtain a semicover $N$ of $M$.

Note that $\partial N$ is the union of all null colored tori. By Corollary 4.5, there is a finite cover $\tilde{N}$ of $N$ which embeds into a regular finite cover $\tilde{M}$. We decompose $\tilde{N}$ by elevations of the elevated colored chunks that compose $N$, and regard any JSJ piece of $\tilde{M}$ not contained in $\tilde{N}$ as an elevated null colored JSJ piece. Then $\tilde{M}$ is as desired.

5.2. Constructing the virtual representation. We use the construction from the previous subsection to find a virtual extension of the representation in the assumption of Theorem 5.2. As in the assumption of Theorem 5.2, let $M$ be an orientable closed mixed 3-manifold, and suppose

$$
\rho_{0}: \pi_{1}\left(J_{0}\right) \rightarrow \mathscr{G}
$$

is a representation of the fundamental group of a geometric piece $J_{0}$ in a Lie group $\mathscr{G}$, which restricted to each boundary component has nontrivial kernel. We also suppose that 
the images of $\rho$ restricted to the boundary components yield a class invertible collection of abelian subgroups of $\mathscr{G}$. Fix a representative automorphism of a class inversion

$$
\nu: \mathscr{G} \rightarrow \mathscr{G}
$$

with respect to this collection.

Let $T_{1}, \cdots, T_{s}$ be the components of $\partial J_{0}$, and let

$$
\zeta_{i} \subset T_{i}
$$

be a slope killed by $\rho_{0}$, for each $1 \leq i \leq s$. By the construction of Subsection 5.1 , there is a regular finite cover

$$
\kappa: \tilde{M} \rightarrow M
$$

obtained by gluing elevated colored chunks matching the coloring (Lemma 5.3).

Lemma 5.4. With the notations above, there is a representation

$$
\tilde{\rho}: \pi_{1}(\tilde{M}) \rightarrow \mathscr{G}
$$

satisfying the following:

- for each elevated positively colored $J_{0}$ piece $\tilde{J}_{0} \subset \tilde{M}$, $\tilde{\rho}$ restricted to $\pi_{1}\left(\tilde{J}_{0}\right)$ is conjugate to $\kappa^{*}\left(\rho_{0}\right)$;

- for each elevated negatively colored $J_{0}$ piece $\tilde{J}_{0} \subset \tilde{M}$, $\tilde{\rho}$ restricted to $\pi_{1}\left(\tilde{J}_{0}\right)$ is conjugate to $\nu \circ \kappa^{*}\left(\rho_{0}\right)$;

- for each elevated colored corridor chunk $\tilde{X}_{i}^{*} \subset \tilde{M}$, $\tilde{\rho}$ restricted to $\pi_{1}\left(\tilde{X}_{i}^{*}\right)$ is cyclic; and

- for any elevated null colored JSJ piece of $\tilde{J} \subset \tilde{M}$, $\tilde{\rho}$ restricted to $\pi_{1}(\tilde{J})$ is trivial.

Proof. By Lemma 3.2 (see also Remark 5.5), we need only to construct the local representations with given properties so that they agree on each boundary component up to congugcy.

In the statement of Lemma 5.4, the representations restricted to elevated colored $J_{0}$ pieces and to elevated null colored pieces describe themselves. We explain the representation for elevated colored corridor chunks as follows.

Suppose $\left(X_{i}^{*}, R_{i}^{*}\right)$ is a corridor chunk associated to $\left(T_{i}, \zeta_{i}\right)$. We write the canonical semicovering from $X_{i}^{*}$ to $M$ as

$$
\mu_{i}: X_{i}^{*} \rightarrow M .
$$

Remember that in Subsection 5.1, we have fixed an orientation of the corridor surface $R_{i}^{*}$ for convenience. The oriented properly embedded subsurface $R_{i}^{*}$ represents a class $\left[R_{i}^{*}\right] \in H_{2}\left(X_{i}^{*}, \partial X_{i}^{*} ; \mathbb{Z}\right) \cong H^{1}\left(X_{i}^{*} ; \mathbb{Z}\right)$. Then homological pairing with $\left[R_{i}^{*}\right]$ induces a quotient homomorphism

$$
\phi_{i}: \pi_{1}\left(X_{i}^{*}\right) \longrightarrow H_{1}\left(X_{i}^{*} ; \mathbb{Z}\right) \stackrel{\left[R_{i}^{*}\right]}{\longrightarrow} \mathbb{Z} .
$$

For any positively or negatively colored elevation $T_{i}^{*} \subset \partial X_{i}^{*}$ of $T_{i}$, since $R_{i}^{*}$ meets $T_{i}^{*}$ in exactly one slope, $\phi_{i}$ surjects into $\mathbb{Z}$ when restricted to $\pi_{1}\left(T_{i}^{*}\right)$. Suppose $\gamma_{i}^{*} \subset T_{i}^{*}$ is a directed slope so that $\phi_{i}\left(\left[\gamma_{i}^{*}\right]\right)$ equals 1 in $\mathbb{Z}$. We define a representation $\alpha_{T_{i}^{*}}: \mathbb{Z} \rightarrow \mathscr{G}$, by assigning $\alpha_{T_{i}^{*}}(1)$ to be either $\left(\rho_{0} \circ\left(\mu_{i}\right)_{\sharp}\right)\left(\left[\gamma_{i}^{*}\right]\right)$ or $\left(\nu \circ \rho_{0} \circ\left(\mu_{i}\right)_{\sharp}\right)\left(\left[\gamma_{i}^{*}\right]\right)$, according to $T_{i}^{*}$ being positively or negatively colored, respectively. Note that $\alpha_{T_{i}^{*}}$ is well defined up to conjugacy of $\mathscr{G}$. By the construction of $\left(X_{i}^{*}, R_{i}^{*}\right)$, and in the notations of Subsection 5.1 $X_{i}^{*}$ is the carrier chunk of $R_{i}^{*}$ in a regular finite cover $X_{i}^{\prime \prime}$ of the carrier chunk $X^{\prime}\left(R_{i}^{\prime}\right) \subset$ $M_{i}^{\prime}$, so any two $T_{i}^{*}$ 's differ only by a deck transformation of $X_{i}^{\prime \prime}$ over $X^{\prime}\left(R_{i}^{\prime}\right)$. It follows 
that $\alpha_{T_{i}^{*}}$ up to conjugacy is independent of the choice of $T_{i}^{*}$. In other words, we have a representation:

$$
\alpha_{i}: \mathbb{Z} \rightarrow \mathscr{G}
$$

defined by any $\alpha_{T_{i}^{*}}$. We define

$$
\rho_{i}: \pi_{1}\left(X_{i}^{*}\right) \rightarrow \mathscr{G}
$$

as $\alpha_{i} \circ \phi_{i}$, up to conjugacy. Finally, for an elevated corridor chunk $\tilde{X}_{i}^{*} \subset \tilde{M}$, with the defining covering

$$
\tilde{\kappa}_{i}: \tilde{X}_{i}^{*} \rightarrow X_{i}^{*}
$$

we define

$$
\tilde{\rho}: \pi_{1}\left(\tilde{X}_{i}^{*}\right) \rightarrow \mathscr{G}
$$

as $\rho_{i} \circ\left(\tilde{\kappa}_{i}\right)_{\sharp}$, up to conjugacy.

We must check that the representation $\left.\tilde{\rho}\right|_{\pi_{1}\left(\tilde{X}_{i}^{*}\right)}$ agrees with the adjacent representations up to conjugacy along the boundary. Note that $\tilde{X}_{i}^{*}$ is only adjacent to elevated null colored JSJ pieces and elevated colored $J_{0}$ pieces. If $\tilde{T} \subset \partial \tilde{X}_{i}^{*}$ is null colored, this means that under $\kappa_{i}, \tilde{T}$ covers a boundary torus of $X_{i}^{*}$ that misses $\partial R_{i}^{*}$. Then $\tilde{\rho}$ is trivial restricted to $\tilde{T}$, and it agrees with the trivial representation $\tilde{\rho}$ on the adjacent elevated null colored piece. If $\tilde{T} \subset \partial \tilde{X}_{i}^{*}$ is positively or negatively colored, it follows from the definition of $\alpha_{i}$ that $\rho_{i}$ restricted to each $\pi_{1}\left(T_{i}^{*}\right)$ is conjugate to the restriction of $\rho_{0}$ or $\nu \circ \rho_{0}$ according to the coloring. Thus $\left.\tilde{\rho}\right|_{\pi_{1}\left(\tilde{X}_{i}^{*}\right)}$ is also conjugate to the restriction of $\rho_{0}$ or $\nu \circ \rho_{0}$ to $\pi_{1}(\tilde{T})$ according to the coloring, since $\left.\tilde{\rho}\right|_{\pi_{1}\left(\tilde{X}_{i}^{*}\right)}$ is the pull back of $\rho_{i}$ via the subgroup inclusion $\left(\tilde{\kappa}_{i}\right)_{\sharp}$.

Because every elevated positively or negatively colored $J_{0}$ piece is only adjacent to corridor chunks, and because $\tilde{\rho}$ trivially agrees along a torus adjacent to two elevated null colored pieces, we have verified that the $\tilde{\rho}$ we have defined on the elevated color chunks of $\tilde{M}$ agree up to conjugacy on the tori that they glue up along. We conclude that there is a representation $\tilde{\rho}: \pi_{1}(\tilde{M}) \rightarrow \mathscr{G}$, as desired.

Lemma 5.4 implies Theorem 5.2, so we have completed the proof of Theorem 5.2 .

Remark 5.5. Note our $\pi_{1}(\tilde{M})$ isomorphic the fundamental group of the graph-of-groups induced by the obvious graph-of-spaces decomposition, canonical up to choosing base points of vertex spaces and paths to base points of adjacent edge spaces, and up to choosing a base point of $\tilde{M}$ and paths to the base points of vertex spaces, cf. [Se].

In general, we can glue up representations on vertex groups as long as they agree on the edge groups up to conjugacy. This is a consequence of the following facts. If $\Gamma=\Gamma_{1} *_{H} \Gamma_{2}$ is an amalgamation of groups, and if $\rho_{i}: \Gamma_{i} \rightarrow \mathscr{G}$, where $i=1,2$, are representations such that $\left.\rho_{1}\right|_{H}$ are conjugate to $\left.\rho_{2}\right|_{H}$, then there is a representation $\rho: \Gamma \rightarrow \mathscr{G}$. More precisely, suppose $\left.\rho_{1}\right|_{H}=\left.\sigma_{h} \circ \rho_{2}\right|_{H}$, where $\sigma_{h}$ is the conjugation of $h \in \mathscr{G}$, then $\rho$ can be defined by taking $\rho_{1}$ on $\Gamma_{1}$ and $\sigma_{h} \circ \rho_{2}$ on $\Gamma_{2}$. Similarly, if $\Gamma=\Gamma_{0} *_{H}$ is an HNN extension with stable letter $t$, and if $\rho_{0}: \Gamma_{0} \rightarrow \mathscr{G}$ is a representation such that $\left.\rho_{0}\right|_{H}$ is conjugate to $\left.\rho_{0}\right|_{H^{t}}$, say by $\sigma_{h}$, then there is a representation $\rho: \Gamma \rightarrow \mathscr{G}$, for example, defined by taking $\rho_{0}$ on $\Gamma_{0}$ and $\rho(t)=h$.

\section{VOLUME COMPUTATION}

In this section, we prove Theorem 1.6 and Proposition 1.4 using the techniques developed in the previous sections. 
6.1. Virtually positive volume of representations. We apply Theorem 5.2 to prove Theorem 1.6. The lemma below verifies class inversion properties of $\operatorname{PSL}(2 ; \mathbb{C})$ and $\mathrm{Iso}_{e} \mathrm{SL}_{2}(\mathbb{R})$. Moreover, for the discussion about representation volumes, we are particularly interested in whether the class inversions can be realized by conjugation using orientation preserving isomorphisms of the geometric space.

\section{Lemma 6.1.}

(1) $\operatorname{PSL}(2 ; \mathbb{C})$ is class invertible with respect to all its cyclic subgroups, and a class inversion can be realized by an inner automorphism of $\mathrm{PSL}(2 ; \mathbb{C})$, which is orientation preserving acting on $\mathbf{H}^{3}$;

(2) $\mathrm{Iso}_{e} \widehat{\mathrm{SL}_{2}(\mathbb{R})}$ is class invertible with respect to its center $\mathbb{R}$, and a class inversion can be realized by an inner automorphism of $\mathrm{IsoSL}_{2}(\mathbb{R})$, which is orientation preserving acting on $\widetilde{\mathrm{SL}_{2}(\mathbb{R})}$.

Proof. The first statement follows from the fact that every element of $\mathrm{PSL}_{2}(\mathbb{C})$ is conjugate to its inverse in $\mathrm{PSL}_{2}(\mathbb{C})$. To see the second statement, note that $\mathrm{IsoSL}_{2}(\mathbb{R})$ has two components. For any $\nu$ in the non-identity component, conjugating $\mathrm{Iso}_{e} \widetilde{\mathrm{SL}_{2}(\mathbb{R})}$ by $\nu$ sends any $r \in \mathbb{R}$ to $-r \in \mathbb{R}$, so it is a class inversion for $\mathbb{R}$. Recall that there are no orientation reversing isometries in the $\mathrm{SL}_{2}$-geometry.

Proof of Theorem 1.6 We first show the hyperbolic volume case. Suppose $M$ contains at least one hyperbolic piece $J_{0}$. It suffices to prove the theorem when $M$ is mixed. We take sufficiently long slopes, one in each component of $\partial J_{0}$, making sure that Dehn fillings along these slopes yield a closed hyperbolic 3 -manifold $\bar{J}_{0}$ of finite volume. Let $\rho_{0}$ : $\pi_{1}\left(J_{0}\right) \rightarrow \operatorname{PSL}(2 ; \mathbb{C})$ be the representation factoring through the the Dehn filling and the discrete faithful representation of $\pi_{1}\left(\bar{J}_{0}\right)$. By Theorem 5.2, we can virtually extend $\rho_{0}$ to $\rho: \pi_{1}(\tilde{M}) \rightarrow \operatorname{PSL}(2 ; \mathbb{C})$. Moreover, it follows from the conclusion of Theorem 5.2 and the additivity principle (Theorem 1.11) and Lemma 3.6 that only some elevations of $J_{0}$ could contribute to the hyperbolic representation volume of $\tilde{M}$. By Lemma 6.1 (1) the volume of all these elevations is a positive multiple of the hyperbolic volume of $\bar{J}_{0}$. Thus the hyperbolic representation volume of $\tilde{M}$ is positive.

It remains to show the Seifert volume case. Since the theorem is known for graph manifolds [DW] and and for geometric manifolds [BG1], we may again assume $M$ to be mixed. By the assumption, $M$ also contains a Seifert geometric piece $J_{0}$. The rest of the argument is almost the same as the previous case, except that: we start by picking a slope $\zeta_{i} \subset T_{i}$ which intersects the Seifert fiber $t_{i} \subset T_{i}$ exactly once for each component $T_{i}$ of $\partial J_{0}$, moreover those $\zeta_{i}$ can be chosen so that the Dehn filling $\bar{J}_{0}$ of $J_{0}$ has a nontrivial Euler class. Then we can choose $\left[t_{i}\right]$ to be the $\gamma_{i}^{*}$ in the proof of Lemma 5.4 and applying Theorem 5.2. Lemma 6.1 2), and Theorem 1.11, we will find a finite cover $\tilde{M}$ with positive Seifert volume.

6.2. Volumes of representations of Seifert manifolds. Now we will prove Proposition 1.4.

Let $N$ be a closed oriented $\widetilde{\mathrm{SL}_{2}(\mathbb{R})}$-manifold whose base 2-orbifold is an orientable, hyperbolic 2 -orbifold $\mathcal{O}$ with positive genus $g$ and $p$ singular points. Then, keeping the same notation as in section 2.3 , we have a presentation

$$
\begin{gathered}
\pi_{1} N=\left\langle\alpha_{1}, \beta_{1}, \ldots, \alpha_{g}, \beta_{g}, s_{1}, \ldots, s_{p}, h:\right. \\
\left.s_{1}^{a_{1}} h^{b_{1}}=1, \ldots, s_{p}^{a_{p}} h^{b_{p}}=1,\left[\alpha_{1}, \beta_{1}\right] \ldots\left[\alpha_{g}, \beta_{g}\right]=s_{1} \ldots s_{p}\right\rangle
\end{gathered}
$$


with the condition $e=\sum_{i} b_{i} / a_{i} \neq 0$. The following result of Eisenbud-Hirsch-Neumann [EHN], which extends the result of Milnor-Wood [Mi, Wo] from circle bundles to Seifert manifolds, is very useful for our purpose.

Theorem 6.2 ([EHN Theorem 3.2 and Corollary 4.3]). Suppose $N$ is a closed orientable Seifert manifold with a regular fiber $h$ and base of genus $>0$.

(1) There is a $\left(\mathrm{PSL}_{2}(\mathbb{R}), \mathbf{S}^{1}\right)$ horizontal foliation on $N$ if and only if there is a representation $\widetilde{\phi}: \pi_{1}(N) \rightarrow \widetilde{\mathrm{SL}_{2}(\mathbb{R})}$ such that $\widetilde{\phi}(h)=\operatorname{sh}(1)$;

(2) Suppose $N=\left(g, 0 ; a_{1} / b_{1}, \ldots, a_{n} / b_{n}\right)$, then there is a $\left(\mathrm{PSL}_{2}(\mathbb{R}), \mathbf{S}^{1}\right)$ horizontal foliation on $N$ if and only if

$$
\sum\left\llcorner b_{i} / a_{i}\right\lrcorner \leq-\chi\left(F_{g}\right) ; \quad \sum\left\ulcorner b_{i} / a_{i}\right\urcorner \geq \chi\left(F_{g}\right)
$$

In order to prove Proposition 1.4 we will check the following proposition which describes those representations leading to a non zero volume. For each element $(a, b) \in$ $\mathbb{R} \times \widetilde{\mathrm{SL}_{2}(\mathbb{R})}$, its image in $\mathbb{R} \times{ }_{\mathbb{Z}} \widetilde{\mathrm{SL}_{2}(\mathbb{R})}$ will be denoted as $\overline{(a, b)}$.

Proposition 6.3. A representation $\rho: \pi_{1}(N) \rightarrow \mathrm{Iso}_{e} \widetilde{\mathrm{SL}_{2}(\mathbb{R})}=\mathbb{R} \times_{\mathbb{Z}} \widetilde{\mathrm{SL}_{2}(\mathbb{R})}$ has nonzero volume if and only if there are integers $n, n_{1}, \ldots, n_{p}$ subject to the conditions

$$
\sum\left\llcorner n_{i} / a_{i}\right\lrcorner-n \leq 2 g-2 \text { and } \sum\left\ulcorner n_{i} / a_{i}\right\urcorner-n \geq 2-2 g
$$

such that

$$
\rho\left(s_{i}\right)=\overline{\left(\frac{n_{i}}{a_{i}}-\frac{b_{i}}{a_{i}} \frac{1}{e}\left(\sum_{i}\left(\frac{n_{i}}{a_{i}}\right)-n\right), g_{i} \operatorname{sh}\left(\frac{-n_{i}}{a_{i}}\right) g_{i}^{-1}\right)}
$$

where $g_{i}$ is an element of $\widetilde{\mathrm{SL}_{2}(\mathbb{R})}$ and

$$
\rho(h)=\overline{\left(\frac{1}{e}\left(\sum_{i}\left(\frac{n_{i}}{a_{i}}\right)-n\right), 1\right)}
$$

whose volume is given by

$$
\operatorname{vol}(N, \rho)=4 \pi^{2} \frac{1}{|e|}\left(\sum_{i}\left(\frac{n_{i}}{a_{i}}\right)-n\right)^{2}
$$

Moreover the $\rho$-image of $\alpha_{1}, \beta_{1}, \ldots, \alpha_{g}, \beta_{g}$ can be chosen to lie in $\widetilde{\mathrm{SL}_{2}(\mathbb{R})}$.

Proof. The condition $\operatorname{vol}(N, \rho) \neq 0$ implies that $\rho(h)=\overline{(\zeta, 1)} \in G=\mathbb{R} \times_{\mathbb{Z}} \widetilde{\mathrm{SL}_{2}(\mathbb{R})}$ by [BG1, p. 663] and [BG2, p. 537], using a cohomological-dimension argument and the definition in paragraph 2.2. Suppose $\rho\left(s_{i}\right)=\overline{\left(z_{i}, x_{i}\right)}$. Then $s_{i}^{a_{i}} h^{b_{i}}=1$ implies that

$$
\overline{\left(a_{i} z_{i}, x^{a_{i}}\right)\left(b_{i} \zeta, 1\right)}=\overline{\left(a_{i} z_{i}+b_{i} \zeta, x^{a_{i}}\right)}=1 .
$$

Then there is an $n_{i} \in \mathbb{Z}$ such that (see Remark 2.3)

$$
a_{i} z_{i}+b_{i} \zeta_{i} \text { in } \mathbb{R} \text { and } x_{i} \text { is conjugate in } \widetilde{\mathrm{SL}_{2}(\mathbb{R})} \text { to } \operatorname{sh}\left(-\frac{n_{i}}{a_{i}}\right) .
$$


Since $\left[\alpha_{1}, \beta_{1}\right] \ldots\left[\alpha_{g}, \beta_{g}\right]=s_{1} \ldots s_{p}$ and since the product of commutators in $\mathbb{R} \times_{\mathbb{Z}}$ $\widetilde{\mathrm{SL}_{2}(\mathbb{R})}$ must lie in $\widetilde{\mathrm{SL}_{2}(\mathbb{R})}$ this implies that

$$
\overline{\left(z_{1}+\ldots+z_{p}, x_{1} \ldots x_{p}\right)}=\overline{\left(0, \prod_{j=1}^{g}\left[\rho\left(\alpha_{j}\right), \rho\left(\beta_{j}\right)\right]\right)}
$$

Then there is an $n \in \mathbb{Z}$ such that

$$
z_{1}+\ldots+z_{p} \text { and } \prod_{j=1}^{g}\left[\rho\left(\alpha_{j}\right), \rho\left(\beta_{j}\right)\right]=x_{1} \ldots x_{p} \operatorname{sh}(n)
$$

Equalities (6.6) and (6.5), imply condition (6.1) in Proposition 6.3 using Theorem 6.2 and its proof in [EHN]. By (6.5) and (6.6), we can calculate directly

$$
z_{i}=\frac{n_{i}}{a_{i}}-\frac{b_{i}}{a_{i}} \zeta, \quad \zeta=\frac{1}{e}\left(\sum_{i=1}^{p} \frac{n_{i}}{a_{i}}-n\right)
$$

Plugging (6.5), (6.6) and (6.7) into $\rho(h)=(\zeta, 1)$ and $\rho\left(s_{i}\right)=\left(z_{i}, x_{i}\right)$, we obtain (6.2) and (6.3) in Proposition 6.3. Then the "moreover" part of Proposition 6.3 also follows from Theorem 6.2

Let's now compute the volume of such a representation. Let $p_{1}: \widetilde{N} \rightarrow N$ be a covering from a circle bundle $\widetilde{N}$ over $\widetilde{F}$ to $N$ so that the fiber degree is 1 . Then we have

$$
\widetilde{e}=e(\widetilde{N})=\left(\operatorname{deg} p_{1}\right) e .
$$

Let $\widetilde{t}$ be the fiber of $\widetilde{N}$ and $\widetilde{\rho}=\rho \mid \pi_{1} \widetilde{N}$. Then $(\widetilde{t})^{\widetilde{e}}=\prod_{j=1}^{\widetilde{g}}\left[\widetilde{\alpha_{j}}, \widetilde{\beta}_{j}\right]$ in $\pi_{1} \widetilde{N}$, and therefore $\widetilde{\rho}\left((\widetilde{t})^{\widetilde{e}}\right)=(\overline{\widetilde{e} \zeta, 1}) \in Z(G) \cap \widetilde{\mathrm{SL}_{2}(\mathbb{R})}$, since the image of the fiber must be in the center and the image of the product of commutators must lie in $\widetilde{\mathrm{SL}_{2}(\mathbb{R})}$. Hence $\widetilde{e} \zeta=\widetilde{n} \in \mathbb{Z}$.

Let $p_{2}: \widehat{N} \rightarrow \widetilde{N}$ be the covering along the fiber direction of degree $\widetilde{e}$, and then $\widehat{e}=$ $e(\widehat{N})=1$. Then $\widehat{\rho}=\widetilde{\rho} \mid$ sends actually $\pi_{1} \widehat{N}$ into $\widehat{\mathrm{SL}_{2}(\mathbb{R})}$ and the fibre $\widehat{t}$ of $\widehat{N}$ is sent to $\operatorname{sh}(\widetilde{n})$. Finally there is a covering $p_{*}: \widehat{N} \rightarrow N^{*}$ along the fiber direction of degree $\widetilde{n}$, where $N^{*}$ is a circle bundle over a hyperbolic surface $F$ with $e^{*}=e\left(N^{*}\right)=\widetilde{n}$. It is apparent that $\hat{\rho}$ descends to $\rho^{*}: \pi_{1} N^{*} \rightarrow \widetilde{\mathrm{SL}_{2}(\mathbb{R})}$ such that $\rho^{*}\left(h^{*}\right)=\operatorname{sh}(1)$, where $h^{*}$ denotes the $\mathbf{S}^{1}$-fiber of $N^{*}$. According to Theorem 6.2 there is $\left(\mathrm{PSL}_{2}(\mathbb{R}), \mathbf{S}^{1}\right)$-horizontal foliation on $N^{*}$, and according to Proposition 2.4 $\operatorname{vol}\left(N^{*}, \rho^{*}\right)=4 \pi^{2} e^{*}=4 \pi^{2} \widetilde{n}$, and then

$$
\operatorname{vol}(\widehat{N}, \widehat{\rho})=4 \pi^{2} \widetilde{n}^{2}=4 \pi^{2} \widetilde{e}^{2} \zeta^{2} .
$$

Note that

$$
\operatorname{deg} p_{1} \operatorname{deg} p_{2}=\frac{\widetilde{e}}{e} \times \widetilde{e}=\frac{\widetilde{e}^{2}}{e} .
$$

By those facts we reach (6.4) as below:

$$
\operatorname{vol}(N, \rho)=\frac{\operatorname{vol}(\widehat{N}, \widehat{\rho})}{\operatorname{deg} p_{1} \operatorname{deg} p_{2}}=\frac{4 \pi^{2} \widetilde{e}^{2} \zeta^{2}}{\frac{\widetilde{e}^{2}}{e}}=4 \pi^{2} e \zeta^{2}=\frac{4 \pi^{2}}{|e|}\left(\sum_{i=1}^{p} \frac{n_{i}}{a_{i}}-n\right)^{2} .
$$


Remark 6.4. Suppose in Proposition 6.3 that $n_{i}=a_{i} k_{i}+r_{i}$, where $0 \leq r_{i}<a_{i}$. If we choose $n=2-2 g+\sum_{i} k_{i}$ and $n_{i}=\left(k_{i}+1\right) a_{i}-1$ then the corresponding representation $\rho_{0}$ is faithful, discrete and reaches the maximal volume giving rise to the well known formula

$$
\operatorname{vol}\left(N, \rho_{0}\right)=\frac{4 \pi^{2} \chi_{O(N)}^{2}}{|e(N)|}
$$

\section{VOLUMES OF REPRESENTATIONS DO NOT HAVE THE COVERING PROPERTY}

In this section, we prove Theorem 1.7 and therefore Corollary 1.8 They follow immediately from the two propositions of this section.

\subsection{Non-trivial graph manifolds with vanishing Seifert volume.}

Proposition 7.1. There are infinitely many non-trivial graph manifolds with zero Seifert volume.

We begin with an elementary lemma in $\widetilde{\mathrm{SL}_{2}(\mathbb{R})}$ geometry. Recall Lemma 2.3 .

Lemma 7.2. Let $\Gamma$ be a subgroup of $G$ and denote by $\bar{\Gamma}$ its projection onto $\operatorname{PSL}(2 ; \mathbb{R})$. If $\bar{\Gamma}$ is abelian then so is $\Gamma$.

Proof. This follows from [EHN, Lemma 2.1]. Let $g=\overline{(\xi, x)}$ and $h=\overline{(\eta, y)}$ be two elements of $\Gamma$. Then note that $[g, h]=\overline{(0,[x, y])}=[x, y]$ is actually a commutator in $\widehat{\mathrm{SL}_{2}(\mathbb{R})}$. If $\bar{\Gamma}$ is abelian then $[g, h]$ belongs to $\mathbb{R} \cap \widehat{\mathrm{SL}_{2}(\mathbb{R})}=\mathbb{Z}$ and there exists an integer $k$ such that $[x, y]=\operatorname{sh} k$.

Recall that $x$ can be seen as a homeomorphism of the real line and using the notations of [EHN] we set $m(z)=\min _{z \in \mathbb{R}} x(z)-z$ and $M(z)=\max _{z \in \mathbb{R}} x(z)-z$. Notice that since $x$ is a lifting of an orientation preserving homeomorphism of the circle then $x(z+1)=x(z)+1$ and these min and max can be considered only on $[0,1]$ so that the definition makes sense.

Besides by [EHN, Lemma 2.1(5)] $\left\llcorner m\left(x y x^{-1}\right)\right\lrcorner=\llcorner m(y)\lrcorner$ and $\left\ulcorner M\left(x y x^{-1}\right)\right\urcorner=$ $\ulcorner M(y)\urcorner$. Since $x y x^{-1}=\operatorname{sh}(k) . y$ we have by [EHN] Lemma 2.1(4)] and the first equality

$$
k+m(y) \leq m(\operatorname{sh}(k) y)=m\left(x y x^{-1}\right)
$$

and then

$$
k+\llcorner m(y)\lrcorner \leq\llcorner m(\operatorname{sh}(k) y)\lrcorner=\left\llcorner m\left(x y x^{-1}\right)\right\lrcorner=\llcorner m(y)\lrcorner,
$$

and by [EHN, Lemma 2.1(4)] and the second equality

$$
k+M(y) \geq M(\operatorname{sh}(k) y)=M\left(x y x^{-1}\right),
$$

and then

$$
k+\ulcorner M(y)\urcorner \geq\ulcorner M(\operatorname{sh}(k) y)\urcorner=\left\ulcorner M\left(x y x^{-1}\right)\right\urcorner=\ulcorner M(y)\urcorner .
$$

This forces $k=0$ and therefore $[g, h]=[x, y]=1$. This proves the lemma.

Proof of Proposition 7.1] The proof follows from a construction in Motegi [Mo]. We recall it. Let $\left(p_{1}, q_{1}\right)$ and $\left(p_{2}, q_{2}\right)$ be two pairs of co-prime integers and consider $E_{1}$ and $E_{2}$ the orientable Seifert manifolds over a 2-disk with two exceptional fibres whose fundamental groups are given by

$$
\pi_{1} E_{1}=\left\langle c_{1}, c_{2}, t_{1},\left[c_{1}, t_{1}\right]=\left[c_{2}, t_{1}\right]=1, c_{1}^{p_{1}}=t_{1}^{r_{1}}, c_{2}^{q_{1}}=t_{1}^{s_{1}}\right\rangle
$$

and

$$
\pi_{1} E_{2}=\left\langle d_{1}, d_{2}, t_{2},\left[d_{1}, t_{2}\right]=\left[d_{2}, t_{2}\right]=1, d_{1}^{p_{2}}=t_{2}^{r_{2}}, d_{2}^{q_{2}}=t_{2}^{s_{2}}\right\rangle
$$


These are the exterior of two torus knots whose meridians are denoted by $m_{1}$ and $m_{2}$. Notice that $E_{i}$ is Euclidean if and only if $p_{i}=q_{i}=2$ and otherwise it is an $\mathbf{H}^{2} \times$ $\mathbb{R}$-manifold. The couples $\left(m_{1}, t_{1}\right)$ and $\left(m_{2}, t_{2}\right)$ provide a basis of $H_{1}\left(\partial E_{1} ; \mathbb{Z}\right)$ and of $H_{1}\left(\partial E_{2} ; \mathbb{Z}\right)$ and Motegi constucted a closed graph manifold $M$ from $E_{1}$ and $E_{2}$ via an orientation reversing identification $\varphi: \partial E_{1} \rightarrow \partial E_{2}$ sending $t_{1}$ to $m_{2}$ and $m_{1}$ to $t_{2}$.

In [Mo, Section 3] Motegi checked that $H_{1}(M ; \mathbb{Z})$ is isomorphic to $\mathbb{Z} /\left(p_{1} p_{2} q_{1} q_{2}-1\right) \mathbb{Z}$ and that any representation of $\pi_{1} M$ into $\operatorname{PSL}(2 ; \mathbb{C})$ is abelian. Hence for any representation $\rho: \pi_{1} M \rightarrow G, \overline{\rho\left(\pi_{1} M\right)} \subset \operatorname{PSL}(2 ; \mathbb{R}) \subset \operatorname{PSL}(2 ; \mathbb{C})$ must be abelian. By Lemma $7.2 \rho\left(\pi_{1} M\right) \subset G$ must be abelian and since $H_{1}(M ; \mathbb{Z})$ is finite then so is the image $\rho\left(\pi_{1} M\right) \subset G$. This proves $\mathrm{SV}(M)=0$ by Lemma 3.6. To complete the proof of the proposition notice that $M$ is a non-trivial graph manifold if and only if $\left|H_{1}(M ; \mathbb{Z})\right|=p_{1} p_{2} q_{1} q_{2}-1>15$.

\subsection{Mixed 3-manifolds with vanishing hyperbolic volume.}

Proposition 7.3. There are infinitely many 3-manifolds $N$ with non-vanishing $\|N\|$ but $\operatorname{vol}(N, \operatorname{PSL}(2 ; \mathbb{C}))=\{0\}$.

Proof. We first begin by constructing a closed mixed 3-manifold with one hyperbolic piece adjacent to one Seifert piece whose hyperbolic volume vanishes. Let $M_{1}$ denote $F \times \mathbf{S}^{1}$ where $F$ is a surface with positive genus and connected boundary. There is a natural section-fiber basis $(s, h) \subset \partial M_{1}$. On the other hand, it follows from [HM] that there are infinitely many one cusped, complete, finite volume hyperbolic manifolds $M_{2}$ endowed with a basis $(\mu, \lambda) \subset \partial M_{2}$ such that both $M_{2}(\lambda)$ and $M_{2}(\mu)$ have zero simplicial volume (because they are actually connected sums of lens spaces). Denote by $\varphi: \partial M_{1} \rightarrow \partial M_{2}$ the homeomorphism defined by $\varphi(s)=\mu$ and $\varphi(h)=\lambda^{-1}$. Let $M_{\varphi}=M_{1} \cup_{\varphi} M_{2}$. Then $M_{\varphi}$ is a mixed manifold. Denote $\mathcal{T}_{M_{\varphi}}$ by $T$.

Let $\rho: \pi_{1} M_{\varphi} \rightarrow \operatorname{PSL}(2 ; \mathbb{C})$ be any representation and denote by $A$ the resulting connection over $M_{\varphi}$. Notice that either $\rho(s)$ or $\rho(h)$ is trivial. Indeed if $\rho(h) \neq 1$, its centralizer $Z(\rho(h))$ must be abelian in $\operatorname{PSL}(2 ; \mathbb{C})$. Since $h$ is central in $\pi_{1} M_{1}$, this means that $\rho\left(\pi_{1} M_{1}\right)$ is abelian. Since $s$ is homologically zero in $M_{1}$, then $\rho(s)=1$.

Let $\zeta$ be either $s$ or $h$ so that $\rho(\zeta)=1$. After putting $A$ in normal form with respect to $T$, denote by $A_{1}$ and $A_{2}$ the flat connections over $M_{1}$ and $M_{2}$ respectively. Since $\rho(\zeta)$ is trivial then $A_{1}$ and $A_{2}$ do extend over $M_{1}(\zeta)$ and $M_{2}(\zeta)$ to flat connections $\widehat{A}_{1}$ and $\widehat{A}_{2}$, and thus

Eventually taking the imaginary part we get

$$
\mathfrak{c s}_{M_{\varphi}}(A)=\mathfrak{c s}_{M_{1}(\zeta)}\left(\widehat{A}_{1}\right)+\mathfrak{c s}_{M_{2}(\zeta)}\left(\widehat{A}_{2}\right) .
$$

$$
\operatorname{vol}\left(M_{\varphi}, \rho\right)=\operatorname{vol}\left(M_{1}(\zeta), \widehat{\rho}_{1}\right)+\operatorname{vol}\left(M_{2}(\zeta), \widehat{\rho}_{2}\right)
$$

where $\widehat{\rho}_{i}$ denotes the extension of $\rho \mid \pi_{1} M_{i}$ to $\pi_{1} M_{i}(\zeta)$. Since both $\operatorname{vol}\left(M_{1}(\zeta), \widehat{\rho}_{1}\right)$ and $\operatorname{vol}\left(M_{2}(\zeta), \widehat{\rho}_{2}\right)$ do vanish, the proof of Proposition 7.3 is complete.

\section{Conclusions}

In conclusion, given a geometrically meaningful representation of a 3-manifold group, Chern-Simons theory can be applied to compute the associated volume. On the other hand, recent results about separability of surface subgroups are powerful in constructing interesting virtual representations of 3-manifold groups. However, a shortcoming of our approach seems to be that we are not able to control the degree of the cover that we need to pass to, so, for instance, we do not have lower bound estimations of the growth of virtual volumes of representations. 
We propose two further problems.

Problem 8.1. Estimate the growth of virtual hyperbolic volume and virtual Seifert volume.

Since the hyperbolic volume is bounded by the simplicial volume, it has at most linear growth as $\operatorname{HV}(\tilde{M}) /[\tilde{M}: M]$ is bounded by $\mu_{3}\|M\|$. However, we do not know whether Seifert volume has at most linear growth as well.

Problem 8.2. Is the Seifert volume of a closed prime 3-manifold virtually positive if it has positive simplicial volume?

The open case is when the 3-manifold has only hyperbolic pieces in its geometric decomposition.

\section{REFERENCES}

[Ag] I. Agol, The virtual Haken conjecture, with an appendix by I. Agol, D. Groves, J. Manning, preprint, 2012, 32 pages, arXiv: 1204.2810.

[BCG] G. Besson, G. Courtois, S. Gallot, Inégalités de Milnor Wood géométriques, Comment. Math. Helv. 82 (2007), 753-803.

[BG1] R. Brooks, W. Goldman, The Godbillon-Vey invariant of a transversely homogeneous foliation, Trans. Amer. Math. Soc. 286 (1984), no. 2, 651-664.

[BG2] _ Volumes in Seifert space, Duke Math. J. 51 (1984), no. 3, 529-545.

[CS] S.-S. Chern, J. Simons, Characteristic forms and geometric invariants, Ann. of Math. (2) 99 (1974), 48-69.

[DSW] P. Derbez, H.-B. Sun, S.-C. Wang, Finiteness of mapping degree sets for 3-manifolds, Acta Math. Sin. (Engl. Ser.) 27 (2011), no. 5, 807-812.

[DW] P. Derbez, S.-C. Wang, Graph manifolds have virtually positive Seifert voume, J. London Math. Soc. 86 (2012), 17-35.

[Du1] J. L. Dupont, Simplicial de Rham cohomology and characteristic classes of flat bundles, Topol. 15 (1976), no. 3, 233-245.

[Du2] Curvature and Characteristic Classes, Lecture Notes in Mathematics, Vol. 640, SpringerVerlag, Berlin-New York, 1978.

[EHN] D. Eisenbud, U. Hirsch, W. Neumann, Transverse foliations of Seifert bundles and self homeomorphism of the circle, Comment. Math. Helv. 56 (1981), no. 4, 638-660.

[GV] C. Godbillon, J. Vey, Un invariant des feuilletages de codimension 1, (French) C. R. Acad. Sci. Paris Sér. A-B 273 (1971), A92-A95.

[Gr] M. Gromov, Volume and bounded cohomology, Inst. Hautes Études Sci. Publ. Math. 56, (1982), 5-99.

[Ho] G. Hochschild, The Structure of Lie Groups, Holden-Day, Inc., San Francisco-London-Amsterdam, 1965.

[HM] J. A. Hoffman, D. Matignon, Examples of bireducible Dehn fillings, Pac. J. Math., 209, (2003), no. 1, 67-83.

[HW] F. Haglund, D. T. Wise, Special cube complexes, Geom. Funct. Anal. 17 (2008), 1551-1620.

[JS] W. Jaco, P. B. Shalen, Seifert fibered space in 3-manifolds, Mem. Amer. Math. Soc. 21 (1979).

[Joh] K. Johannson Homotopy Equivalence of 3-Manifolds with Boundary, Lecture Notes in Math. 761, Springer-Verlag, Berlin, 1979.

[Ki] R. Kirby, Problems in low-dimensional topology, Geometric Topology, Edited by H. Kazez, AMS/IP, Vol. 2, International Press, 1997.

[Kh] V.-T. Khoi, A cut-and-paste method for computing the Seifert volumes, Math. Ann. 326 (2003), no. 4, 759-801.

[KK] P. Kirk, E. Klassen, Chern-Simons invariants of 3-manifolds decomposed along tori and the circle bundle over the representation space of $T^{2}$, Comm. Math. Phys. 153 (1993), no. 3, 521-557.

[KN] S. Kobayashi, K. Nomizu, Foundations of Differential Geometry, Vol. I. and II., reprint of the 1963 original, John Wiley \& Sons, Inc., New York, 1996.

[Lu] J. Luecke, Finite covers of 3-manifolds containing essential tori, Trans. Amer. Math. Soc. 310 (1984), no. 1, 381-391.

[LW] J. Luecke, Y.-Q. Wu, Relative Euler number and finite covers of graph manifolds, Geometric Topology (Athens, GA, 1993), pp. 80-103, Amer. Math. Soc., Providence, RI, 1997. 
[Mi] J. Milnor On the existence of a connection with curvature zero, Comment. Math. Helv. 32 1958, 215223.

[MT] J. Milnor, W. P. Thurston, Characteristic numbers of 3-manifolds, Enseignement Math. (2) 23 (1977), no. 3-4, 249-254.

[Mo] K. Motegi, Haken manifolds and representations of their fundamental groups in SL(2,C), Topol. Appl. 29 (1988), no. 3, 207-212.

[Ne] W. Neumann, Commensurability and virtual fibration for graph manifolds, Topol. 36 (1997), no. 2, 355-378.

[PW1] P. Przytycki, D. T. Wise, Graph manifolds with boundary are virtually special, J. Topol. 2013, arXiv: 1110.3513.

[PW2] Mixed 3-manifolds are virtually special, arXiv:1205.6742.

[PW3] Separability of embedded surfaces in 3-manifolds, Compositio Mathematica, 2014, arXiv: 1210.7298 .

[Re] A. Reznikov, Rationality of secondary classes, J. Diff. Geom. 43 (1996), no. 3, 674-692.

[RW] J. H. Rubinstein, S.-C. Wang, $\pi_{1}$-injective surfaces in graph-manifolds, Comment. Math. Helv. 73 (1998), 499-515.

[Se] J.-P. Serre, Trees, translated from French by J. Stillwell, Springer-Verlag, 1980.

[So] T. Soma, The Gromov invariant of links, Invent. Math. 64 1981, 445-454.

[Th1] W. P. Thurston, The Geometry and Topology of Three-Manifolds, Lecture Notes, Princeton, 1977.

[Th2] L Three dimensional manifolds, Kleinian groups and hyperbolic geometry, Bull. Amer. Math. Soc. 316, 1982, 357-381.

[V] W. Van Est, Une application d'une méthode de Cartan-Leray (French), Indag. Math. 17 (1955), 542544.

[WW] S.-C. Wang, Y.-Q. Wu, Covering invariants and co-Hopficity of 3-manifold groups, Proc. London Math. Soc. (3) 68 (1994), no. 1, 203-224.

[Wi] D. Wise, The structure of groups with quasiconvex hierarchy, preprint, 2011, 186 pages, available at http://www.math.mcgill.ca/wise/papers.html.

[Wo] J. Wood, Bundles with totally disconnected structure group, Comment. Math. Helv. 46 (1971), 257-273.

[Yo] T. Yoshida, The $\eta$-invariant of hyperbolic 3-manifolds, Invent. Math. 81 (1985), no. 3, 473-514.

LATP UMR 7353, 39 RUE Joliot-CURIE, 13453 MARSEILle CEDEX 13

E-mail address: pderbez@gmail.com

Mathematics 253-37, California Institute of Technology, Pasadena, CA 91125, U.S.A.

E-mail address: yliumathecaltech.edu

Department of Mathematics, Peking University, Beijing, China

E-mail address: wangsc@math.pku.edu.cn 